REPRESENTATION THEORY

An Electronic Journal of the American Mathematical Society

Volume 3, Pages 153-222 (July 19, 1999)

S $1088-4165(99) 00073-4$

\title{
SUBREGULAR NILPOTENT REPRESENTATIONS OF LIE ALGEBRAS IN PRIME CHARACTERISTIC
}

\author{
JENS CARSTEN JANTZEN
}

\begin{abstract}
We look in this paper at representations of Lie algebras of simple reductive groups in prime characteristic. We investigate those modules that have a subregular nilpotent $p$-character. In case all roots in the corresponding root system have the same length, we determine all simple modules in generic blocks as well as the Cartan matrices of these blocks. Our results confirm conjectures by Lusztig. We determine in these cases also extension groups between non-isomorphic simple modules. There are similar, somewhat less detailed results on non-generic blocks and the cases with two root lengths.
\end{abstract}

\section{INTRODUCTION}

Let $G$ be a reductive algebraic group over an algebraically closed field $K$ of prime characteristic $p>0$. This paper deals with certain representations of the Lie algebra $\mathfrak{g}$ of $G$. For the purpose of this introduction assume that $G$ is semi-simple and simply connected, that the root system $R$ of $G$ is irreducible, and that $p$ is larger than the Coxeter number $h$ of $R$. (If $R$ is of type $E_{8}$ or $F_{4}$, assume that $p>h+1$; this restriction should be unnecessary, but my proofs require it.)

Each simple $\mathfrak{g}$-module has a $p$-character; that is a linear form $\chi$ on $\mathfrak{g}$ such that all $x^{p}-x^{[p]}-\chi(x)^{p} 1$ with $x \in \mathfrak{g}$ annihilate the module. Here $x^{p}$ is the $p$-th power of $x$ in the universal enveloping algebra $U(\mathfrak{g})$ and $x \mapsto x^{[p]}$ is the $p$-th power map on the Lie $p$-algebra $\mathfrak{g}$. A general result due to Kac and Weisfeiler reduces the problem of describing all simple modules basically to the case where the $p$-character $\chi$ is nilpotent; this means that $\chi$ vanishes on some Borel subalgebra of $\mathfrak{g}$. Due to work by Curtis, Friedlander, Parshall, and Panov one then has a classification of the simple modules in case $\chi$ has a certain special form ("standard Levi form"). For $\chi$ not of this form so far no classification of the corresponding simple modules has been known.

We look in this paper at the case where $\chi$ is subregular nilpotent. Here "subregular" means that the orbit of $\chi$ under the coadjoint action of $G$ has dimension $2(N-1)$ where $2 N=|R|$. A subregular nilpotent $\chi$ has standard Levi form if and only if $R$ has type $A_{n}$ or $B_{n}$. In those two cases I have given a detailed description of the corresponding simple modules in [J2]. For the other types the results in this paper are new. In order to describe them I first need some notation.

Let $T$ be a maximal torus in $G$, let $X$ be the character group of $T$ and $\mathfrak{h}$ the Lie algebra of $T$. Choose a basis $\Pi$ for the root system $R \subset X$. Set $\rho$ equal to half the sum of the positive roots, and let $\alpha_{0}$ denote the unique short root that is

Received by the editors May 3, 1999 and, in revised form, June 9, 1999.

1991 Mathematics Subject Classification. Primary 17B10; Secondary 17B20, 17B45, 17 B50.

(c)1999 American Mathematical Society 
a dominant weight. (If all roots have the same length, then all roots are short, and none are long.) Set $\widetilde{\Pi}=\Pi \cup\left\{-\alpha_{0}\right\}$.

Consider the algebra $U(\mathfrak{g})^{G}$ of $G$-invariants in $U(\mathfrak{g})$. Each $\lambda \in X$ defines a "central character" $\operatorname{cen}_{\lambda}: U(\mathfrak{g})^{G} \rightarrow K$ such that $U(\mathfrak{g})^{G}$ acts via cen ${ }_{\lambda}$ on a highest weight module with highest weight $\lambda$. Fix a subregular nilpotent $\chi$. Denote by $\mathcal{C}_{\lambda}$ for each $\lambda \in X$ the category of all finite dimensional $\mathfrak{g}$-modules $M$ that are annihilated by all $x^{p}-x^{[p]}-\chi(x)^{p} 1$ with $x \in \mathfrak{g}$ and such that $U(\mathfrak{g})^{G}$ acts via cen on all composition factors of $M$.

Assume first that all roots in $R$ have the same length. (This is the case where our results are most complete.) Let $\lambda \in X$ such that $0<\left\langle\lambda+\rho, \alpha^{\vee}\right\rangle\langle p$ for all positive roots $\alpha$. (We denote by $\alpha^{\vee}$ the coroot corresponding to $\alpha$.) Then there are up to isomorphism $|\widetilde{\Pi}|$ simple modules in $\mathcal{C}_{\lambda}$. We can denote them by $L_{\alpha}^{\lambda}$ with $\alpha \in \widetilde{\Pi}$ such that

$$
\operatorname{dim} L_{\alpha}^{\lambda}= \begin{cases}\left\langle\lambda+\rho, \alpha^{\vee}\right\rangle p^{N-1}, & \text { if } \alpha \in \Pi, \\ \left(p-\left\langle\lambda+\rho, \alpha_{0}^{\vee}\right\rangle\right) p^{N-1}, & \text { if } \alpha=-\alpha_{0} .\end{cases}
$$

Define integers $m_{\alpha}$ for all $\alpha \in \widetilde{\Pi}$ by $m_{-\alpha_{0}}=1$ and $\alpha_{0}^{\vee}=\sum_{\beta \in \Pi} m_{\beta} \beta^{\vee}$. Let $Q_{\alpha}^{\lambda}$ denote the projective cover of $L_{\alpha}^{\lambda}$ in $\mathcal{C}_{\lambda}$. Then

$$
\left[Q_{\alpha}^{\lambda}: L_{\beta}^{\lambda}\right]=|W| m_{\alpha} m_{\beta} \quad \text { for all } \alpha, \beta \in \widetilde{\Pi} .
$$

Here $W$ is the Weyl group of $R$ and we write $[M: L]$ for the multiplicity of a simple module $L$ as a composition factor of a module $M$.

If $\chi$ vanishes on the "standard" Borel subalgebra (corresponding to the positive roots), then one can define "baby Verma modules" $Z_{\chi}(\lambda)$. One gets then

$$
\left[Z_{\chi}(\lambda): L_{\alpha}^{\lambda}\right]=m_{\alpha} \quad \text { for all } \alpha \in \widetilde{\Pi} .
$$

The extension group (in $\mathcal{C}_{\lambda}$ ) of two non-isomorphic simple modules is given by

$$
\operatorname{Ext}^{1}\left(L_{\alpha}^{\lambda}, L_{\beta}^{\lambda}\right) \simeq \begin{cases}K, & \text { if }(\alpha, \beta)<0, \\ 0, & \text { if }(\alpha, \beta)=0,\end{cases}
$$

unless $R$ is of type $A_{1}$ where one has to replace $K$ by $K^{2}$. I do not know how big the Ext group is in case $\alpha=\beta$; it will be non-zero in most cases.

The result on the number of simple modules in $\mathcal{C}_{\lambda}$ as well as the formula in (2) for the Cartan matrix confirm conjectures by Lusztig.

If we consider more generally $\lambda \in X$ such that $0 \leq\left\langle\lambda+\rho, \alpha^{\vee}\right\rangle\langle p$ for all positive roots $\alpha$, then the results in (1), (2), and (3) extend if we drop all $L_{\beta}^{\lambda}$ with $\left\langle\lambda+\rho, \beta^{\vee}\right\rangle=0$ and if we replace $|W|$ in (2) by $|W \cdot \lambda|$. (We use here the 'dot action' where $w_{\bullet} \lambda=w(\lambda+\rho)-\rho$.) In types $A_{n}$ and $D_{n}$ we get thus a description of all possible $\mathcal{C}_{\lambda}$ (that depends only on $W \cdot \lambda+p X$ ), while there remain some open problems in type $E_{n}$.

Consider next $R$ of type $B_{n}, C_{n}$, or $F_{4}$. Choose again a weight $\lambda \in X$ such that $0<\left\langle\lambda+\rho, \alpha^{\vee}\right\rangle<p$ for all positive roots $\alpha$. In this case there is one simple module $L_{\alpha}^{\lambda}$ corresponding to each short root $\alpha \in \widetilde{\Pi}$, and one expects that there are two simple modules $L_{\alpha, 1}^{\lambda}$ and $L_{\alpha, 2}^{\lambda}$ corresponding to each long root $\alpha \in \widetilde{\Pi}$. This expectation is known to hold in type $B_{n}$; in types $C_{n}$ and $F_{4}$ I cannot exclude that we get only one simple module corresponding to a given long simple root.

The formulas in (1)-(4) extend to the present situation as long as all roots occurring are short. The dimension formula (1) holds also for the simple modules 
corresponding to long roots. If our expectation holds, then one gets $L_{\alpha, 2}^{\lambda}$ from $L_{\alpha, 1}^{\lambda}$ by twisting with the adjoint action of a suitable element in the centraliser of $\chi$ in $G$. In (2)-(4) one has to make then minor changes in case long roots occur. For example, if $\alpha$ is long, then one should divide the right hand side in (3) by 2 . If $\alpha, \beta$ are long with $(\alpha, \beta)<0$, then one can choose the numbering of the simple modules such that

$$
\operatorname{Ext}^{1}\left(L_{\alpha, i}^{\lambda}, L_{\beta, j}^{\lambda}\right) \simeq \begin{cases}K, & \text { if } i=j \\ 0, & \text { otherwise. }\end{cases}
$$

There are again similar results for all $\lambda \in X$ such that $0 \leq\left\langle\lambda+\rho, \alpha^{\vee}\right\rangle\langle p$ for all positive roots $\alpha$. In types $B_{n}$ and $C_{n}$ these $\lambda$ suffice to understand all possible $\mathcal{C}_{\mu}$.

In type $G_{2}$ our results are much weaker.

Let me briefly describe the main steps in the proof of the results. We first construct a chain of submodules in a baby Verma module. Using the Kac-Weisfeiler conjecture (Premet's theorem) one can show that this is a composition series. (In the case of two root lengths the argument is actually slightly more complicated.) We then show that all composition factors can be described as induced modules from minimal parabolic subalgebras of $\mathfrak{g}$. (These results are contained in Section D and build on earlier work on translation functors and submodules of baby Verma modules in Sections B and C.)

The main problem then is to decide when two factors in our composition series are isomorphic to each other. In order to show that two modules are not isomorphic to each other, we apply suitable translation functors and show that the modules behave differently under them. In order to show that two modules are isomorphic to each other, we use a deformation argument to construct a non-zero homomorphism. This is done in Section F after some preparations in Sections A and E. This is followed by an investigation of the action of the centraliser in $G$ of $\chi$ on the simple modules.

The results on the Cartan matrix and the Ext groups are contained in Sections $\mathrm{G}$ and H. Here we use "translation through the walls" as a main technique.

Much of this work was done while I was visiting the SPIC Mathematical Institute (now: Chennai Mathematical Institute) and the Tata Institute of Fundamental Research. I would like to thank all those who made those stays possible and enjoyable, in particular C. S. Seshadri and K. N. Raghavan, V. B. Mehta and A. J. Parameswaran. I also would like to thank Jim Humphreys for several useful suggestions.

A.

A.1. Let $K$ be an algebraically closed field of characteristic $p>0$. Let $G$ be a reductive algebraic group over $K$ and denote by $\mathfrak{g}$ the Lie algebra of $G$. This is a restricted Lie algebra as the Lie algebra of an algebraic group; we denote the $p$-th-power map by $x \mapsto x^{[p]}$.

Let $T$ be a maximal torus in $G$ and set $\mathfrak{h}=\operatorname{Lie}(T)$. Let $X=X(T)$ be the (additive) group of all characters of $T$ and let $R \subset X$ be the root system of $G$. For each $\alpha \in R$ let $\mathfrak{g}_{\alpha}$ denote the corresponding root subspace of $\mathfrak{g}$. We choose a system $R^{+}$of positive roots. Set $\mathfrak{n}^{+}$equal to the sum of all $\mathfrak{g}_{\alpha}$ with $\alpha>0$ and $\mathfrak{n}^{-}$ equal to the sum of all $\mathfrak{g}_{\alpha}$ with $\alpha<0$. We have then the triangular decomposition 
$\mathfrak{g}=\mathfrak{n}^{+} \oplus \mathfrak{h} \oplus \mathfrak{n}^{-}$of $\mathfrak{g}$. Set $\mathfrak{b}^{+}=\mathfrak{h} \oplus \mathfrak{n}^{+}$. All of these Lie subalgebras $\left(\mathfrak{h}, \mathfrak{n}^{+}, \mathfrak{n}^{-}\right.$, $\mathfrak{b}^{+}$) of $\mathfrak{g}$ are Lie algebras of closed subgroups of $G$ and hence restricted subalgebras of $\mathfrak{g}$.

For each $\alpha \in R$ let $\alpha^{\vee}$ denote the corresponding coroot. Denote then by $s_{\alpha, n}$ (for all $n \in \mathbf{Z}$ ) the (affine) reflection given by $s_{\alpha, n}(\mu)=\mu-\left(\left\langle\mu, \alpha^{\vee}\right\rangle-n\right) \alpha$ for all $\mu \in X$. Write $W$ for the Weyl group (generated by all $s_{\alpha}=s_{\alpha, 0}$ ), and write $W_{p}$ for the affine Weyl group generated by all $s_{\alpha, m p}$ with $m \in \mathbf{Z}$ and $\alpha \in R$. Then $W_{p}$ contains all translations by $p \alpha$ with $\alpha \in R$, and $W_{p}$ is generated by $W$ and these translations.

Each $\mu \in X$ defines by taking the derivative a linear form $d \mu$ on $\mathfrak{h}$. The map $\mu \mapsto d \mu$ yields an embedding of $X / p X$ into $\mathfrak{h}^{*}$. If $\lambda_{1}, \lambda_{2}, \ldots, \lambda_{s}$ is a basis of $X$ over $\mathbf{Z}$, then the $d \lambda_{i}$ are a basis of $\mathfrak{h}^{*}$ over $K$. There exists for each $\alpha \in R$ an element $h_{\alpha} \in \mathfrak{h}$ such that $d \mu\left(h_{\alpha}\right)$ is the reduction modulo $p$ of $\left\langle\mu, \alpha^{\vee}\right\rangle$ for each $\mu \in X$. We choose for each root $\alpha$ a basis vector $x_{\alpha}$ for $\mathfrak{g}_{\alpha}$ such that always $\left[x_{\alpha}, x_{-\alpha}\right]=h_{\alpha}$. We have $x_{\alpha}^{[p]}=0$ and $h_{\alpha}^{[p]}=h_{\alpha}$ for all $\alpha \in R$.

Let $\rho \in X \otimes \mathbf{z} \mathbf{Q}$ be a weight with $\left\langle\rho, \alpha^{\vee}\right\rangle=1$ for all simple roots $\alpha$. We shall use the 'dot action' of $W$ or $W_{p}$ on $X$ given by $w_{\bullet} \lambda=w(\lambda+\rho)-\rho$. (It is independent of the specific choice of $\rho$.)

We denote the universal enveloping algebra of $\mathfrak{g}$ by $U(\mathfrak{g})$. For each $\chi \in \mathfrak{g}^{*}$ set $U_{\chi}(\mathfrak{g})$ equal to the factor algebra of $U(\mathfrak{g})$ by the ideal generated by all $x^{p}-x^{[p]}-\chi(x)^{p}$ with $x \in \mathfrak{g}$. We use analogous notations for restricted subalgebras of $\mathfrak{g}$.

A.2. Each $f \in \mathfrak{h}^{*}$ defines a one dimensional $\mathfrak{h}$-module $K_{f}$ [with each $h \in \mathfrak{h}$ acting as multiplication by $f(h)$ ]. There exists $\chi(f) \in \mathfrak{h}^{*}$ such that $K_{f}$ is a $U_{\chi(f)}(\mathfrak{h})-$ module, i.e., with $\chi(f)(h)^{p}=f(h)^{p}-f\left(h^{[p]}\right)$ for all $h \in \mathfrak{h}$. So we have in particular

$$
\chi(f)\left(h_{\beta}\right)^{p}=f\left(h_{\beta}\right)^{p}-f\left(h_{\beta}\right) \quad \text { for all } \beta \in R
$$

and

$$
f\left(h_{\beta}\right) \in \mathbf{F}_{p} \Longleftrightarrow \chi(f)\left(h_{\beta}\right)=0 .
$$

Any $f_{1}, f_{2} \in \mathfrak{h}^{*}$ satisfy obviously

$$
\chi\left(f_{1}+f_{2}\right)=\chi\left(f_{1}\right)+\chi\left(f_{2}\right) .
$$

We have clearly $\chi(\mu)=0$ for all $\mu \in X$, hence

$$
\chi(f+\mu)=\chi(f) \quad \text { for all } f \in \mathfrak{h}^{*}, \mu \in X .
$$

Here (as usual) we write $\mu$ instead of $d \mu$ by abuse of notation.

For each $\chi \in \mathfrak{g}^{*}$ set

$$
\Lambda_{\chi}=\left\{f \in \mathfrak{h}^{*} \mid \chi_{\mid \mathfrak{h}}=\chi(f)\right\} .
$$

Given $f \in \mathfrak{h}^{*}$ we can regard $K_{f}$ as a $\mathfrak{b}^{+}$-module with $\mathfrak{n}^{+}$acting trivially. This is then a $U_{\chi}\left(\mathfrak{b}^{+}\right)$-module for all $\chi \in \mathfrak{g}^{*}$ satisfying $\chi\left(\mathfrak{n}^{+}\right)=0$ and $f \in \Lambda_{\chi}$. For all these $\chi$ we get then an induced $U_{\chi}(\mathfrak{g})$-module (a "baby Verma module")

$$
Z_{\chi}(f)=U_{\chi}(\mathfrak{g}) \otimes_{U_{\chi}(\mathfrak{b}+)} K_{f} .
$$

Denote its "standard generator" $1 \otimes 1$ by $v_{f}$. As before, we usually write $Z_{\chi}(\lambda)$ instead of $Z_{\chi}(d \lambda)$ for $\lambda \in X$; we should keep in mind that $Z_{\chi}(\lambda)$ depends only on $d \lambda$, hence on the coset $\lambda+p X$.

If $f^{\prime} \in \mathfrak{h}^{*}$ satisfies $f^{\prime}\left(h_{\beta}\right)=0$ for all $\beta \in R$, then we can extend $K_{f^{\prime}}$ to a $\mathfrak{g}-$ module with all $x_{\beta}$ acting as 0 . This is then a $U_{\chi\left(f^{\prime}\right)}(\mathfrak{g})$-module where we extend 
$\chi\left(f^{\prime}\right)$ to a linear form on $\mathfrak{g}$ such that $\chi\left(f^{\prime}\right)\left(\mathfrak{n}^{+}+\mathfrak{n}^{-}\right)=0$. A trivial form of the tensor identity yields then

$$
Z_{\chi}(f) \otimes K_{f^{\prime}} \simeq Z_{\chi+\chi\left(f^{\prime}\right)}\left(f+f^{\prime}\right)
$$

for all $f \in \mathfrak{h}^{*}$ and $\chi \in \mathfrak{g}^{*}$ with $f \in \Lambda_{\chi}$ and $\chi\left(\mathfrak{n}^{+}\right)=0$.

A.3. Fix a simple root $\alpha$ for the rest of Section A. Denote by $\mathfrak{p}_{\alpha}=\mathfrak{b}^{+}+\mathfrak{g}_{-\alpha}$ the corresponding parabolic subalgebra. For all $f \in \mathfrak{h}^{*}$ and $\chi \in \mathfrak{g}^{*}$ with $\chi\left(\mathfrak{n}^{+}\right)=0$ and $f \in \Lambda_{\chi}$ consider the induced $U_{\chi}\left(\mathfrak{p}_{\alpha}\right)$-module

$$
Z_{\chi, \alpha}(f)=U_{\chi}\left(\mathfrak{p}_{\alpha}\right) \otimes_{U_{\chi}\left(\mathfrak{b}^{+}\right)} K_{f}
$$

Denote the "standard generator" $1 \otimes 1$ now by $v_{f}^{\prime}$. It satisfies $x_{\beta} v_{f}^{\prime}=0$ for all $\beta \in R^{+}$and $h v_{f}^{\prime}=f(h) v_{f}^{\prime}$ for all $h \in \mathfrak{h}$; the $x_{-\alpha}^{i} v_{f}^{\prime}$ with $0 \leq i<p$ are a basis of $Z_{\chi, \alpha}(f)$. We have

$$
x_{\alpha}\left(x_{-\alpha}^{i} v_{f}^{\prime}\right)=i\left(f\left(h_{\alpha}\right)+1-i\right) x_{-\alpha}^{i-1} v_{f}^{\prime} \quad \text { for } 0<i<p
$$

and $x_{\beta}\left(x_{-\alpha}^{i} v_{f}^{\prime}\right)=0$ for all $\beta \in R^{+}$with $\beta \neq \alpha$.

Suppose now that

$$
f\left(h_{\alpha}\right) \in \mathbf{F}_{p} .
$$

Let $d$ be the integer with $0<d \leq p$ and $f\left(h_{\alpha}\right)+1=d 1$ in $\mathbf{F}_{p}$. Then $x_{-\alpha}^{d} v_{0}$ is annihilated by all $x_{\beta}$ with $\beta>0$. It follows that we have a homomorphism of $U_{\chi}\left(\mathfrak{p}_{\alpha}\right)$-modules

$$
\varphi_{f}: Z_{\chi, \alpha}(f-d \alpha) \longrightarrow Z_{\chi, \alpha}(f)
$$

given by $\varphi\left(v_{f-d \alpha}^{\prime}\right)=x_{-\alpha}^{d} v_{f}^{\prime}$. [Note that $Z_{\chi, \alpha}(f-d \alpha)$ makes sense because $\chi(f)=$ $\chi(f-d \alpha)$ by A.2(4).]

If $\chi\left(x_{-\alpha}\right) \neq 0$, then $\varphi_{f}$ is an isomorphism since $x_{-\alpha}^{p}$ acts as the scalar $\chi\left(x_{-\alpha}\right)^{p}$ on these modules. If $\chi\left(x_{-\alpha}\right)=0$, then the image of $\varphi_{f}$ is spanned over $K$ by all $x_{-\alpha}^{i} v_{f}^{\prime}$ with $d \leq i<p$. In this case the cokernel of $\varphi_{f}$ [the factor module of $Z_{\chi, \alpha}(f)$ by that image] has dimension $d$; let us denote it by $L_{\chi, \alpha}(f)$. Note that $L_{\chi, \alpha}(f)=Z_{\chi, \alpha}(f)$ in case $d=p$. If $d<p$, then the image of $\varphi_{f}$ is clearly isomorphic to $L_{\chi, \alpha}(f-d \alpha)$.

If $f^{\prime} \in \mathfrak{h}^{*}$ satisfies $f^{\prime}\left(h_{\alpha}\right)=0$, then we can extend $K_{f^{\prime}}$ from a $\mathfrak{b}^{+}$-module to a $\mathfrak{p}_{\alpha}$-module such that $x_{-\alpha}$ acts as 0 . This is then a $U_{\chi\left(f^{\prime}\right)}\left(\mathfrak{p}_{\alpha}\right)$-module where we extend $\chi\left(f^{\prime}\right)$ to a linear form on $\mathfrak{p}_{\alpha}$ such that $\chi\left(f^{\prime}\right)\left(\mathfrak{n}^{+}+\mathfrak{g}_{-\alpha}\right)=0$. We get then [as in A.2(7)] first $Z_{\chi, \alpha}(f) \otimes K_{f^{\prime}} \simeq Z_{\chi+\chi\left(f^{\prime}\right), \alpha}\left(f+f^{\prime}\right)$ and then

$$
L_{\chi, \alpha}(f) \otimes K_{f^{\prime}} \simeq L_{\chi+\chi\left(f^{\prime}\right), \alpha}\left(f+f^{\prime}\right)
$$

whenever $L_{\chi, \alpha}(f)$ is defined.

A.4. Again let $f \in \mathfrak{h}^{*}$ and $\chi \in \mathfrak{g}^{*}$ with $\chi\left(\mathfrak{n}^{+}\right)=0$ and $f \in \Lambda_{\chi}$. We have by transitivity of induction an isomorphism of $U_{\chi}(\mathfrak{g})$-modules

$$
Z_{\chi}(f) \stackrel{\sim}{\longrightarrow} U_{\chi}(\mathfrak{g}) \otimes_{U_{\chi}\left(\mathfrak{p}_{\alpha}\right)} Z_{\chi, \alpha}(f)
$$

that maps $v_{f}$ to $1 \otimes v_{f}^{\prime}$. If $f\left(h_{\alpha}\right) \in \mathbf{F}_{p}$, then we get from A.3 an induced homomorphism of $U_{\chi}(\mathfrak{g})$-modules

$$
\varphi_{\alpha}^{f}: Z_{\chi}(f-d \alpha) \longrightarrow Z_{\chi}(f)
$$

where $d$ is the integer with $0<d \leq p$ and $f\left(h_{\alpha}\right)+1=d 1$ in $\mathbf{F}_{p}$. 
If $\chi\left(x_{-\alpha}\right) \neq 0$, then $\varphi_{\alpha}^{f}$ is again an isomorphism. If $\chi\left(x_{-\alpha}\right)=0$, then we denote the cokernel of $\varphi_{\alpha}^{f}$ by $Z_{\chi}(f, \alpha)$. So we have then an exact sequence

$$
Z_{\chi}(f-d \alpha) \stackrel{\varphi_{\alpha}^{f}}{\longrightarrow} Z_{\chi}(f) \longrightarrow Z_{\chi}(f, \alpha) \rightarrow 0 .
$$

Since induction is exact in our situation, we can identify $Z_{\chi}(f, \alpha)$ with the module induced from the cokernel of $\varphi_{f}$ :

$$
Z_{\chi}(f, \alpha) \simeq U_{\chi}(\mathfrak{g}) \otimes_{U_{\chi}\left(\mathfrak{p}_{\alpha}\right)} L_{\chi, \alpha}(f) .
$$

We have $\operatorname{dim} Z_{\chi}(f, \alpha)=d p^{N-1}$ where $N=\left|R^{+}\right|$. If $d=p$, then $Z_{\chi}(f, \alpha)=Z_{\chi}(f)$. If $d<p$, then the image of $\varphi_{\alpha}^{f}$ is isomorphic to $Z_{\chi}(f-d \alpha, \alpha)$.

We shall use the notation $v_{f}$ also for the image in $Z_{\chi}(f, \alpha)$ of the standard generator of $Z_{\chi}(f)$.

If $f^{\prime} \in \mathfrak{h}^{*}$ satisfies $f^{\prime}\left(h_{\beta}\right)=0$ for all $\beta \in R$, then we get (as at the end of A.2) a $U_{\chi\left(f^{\prime}\right)}(\mathfrak{g})$-module $K_{f^{\prime}}$ where we extend $\chi\left(f^{\prime}\right)$ to a linear form on $\mathfrak{g}$ such that $\chi\left(f^{\prime}\right)\left(\mathfrak{n}^{+}+\mathfrak{n}^{-}\right)=0$. One checks easily that A.2(7) induces an isomorphism

$$
Z_{\chi}(f, \alpha) \otimes K_{f^{\prime}} \simeq Z_{\chi+\chi\left(f^{\prime}\right)}\left(f+f^{\prime}, \alpha\right)
$$

whenever $Z_{\chi}(f, \alpha)$ is defined.

A.5. Let $R^{\prime}$ be a root subsystem of $R$. Then $\mathfrak{g}^{\prime}=\mathfrak{h} \oplus \bigoplus_{\beta \in R^{\prime}} \mathfrak{g}_{\beta}$ the Lie algebra of a reductive closed subgroup of $G$. We can then apply A.2 also to $\mathfrak{g}^{\prime}$ working with $\mathfrak{b}^{+} \cap \mathfrak{g}^{\prime}$ instead of $\mathfrak{b}^{+}$. We shall write then

$$
Z_{\chi}\left(f ; \mathfrak{g}^{\prime}\right)=U_{\chi}\left(\mathfrak{g}^{\prime}\right) \otimes_{U_{\chi}\left(\mathfrak{b}+\cap \mathfrak{g}^{\prime}\right)} K_{f}
$$

for the analogue of $Z_{\chi}(f)$. In case $\alpha \in R^{\prime}$ we can also extend A.3 and A.4 and get

$$
Z_{\chi}\left(f, \alpha ; \mathfrak{g}^{\prime}\right)=U_{\chi}\left(\mathfrak{g}^{\prime}\right) \otimes_{U_{\chi}\left(\mathfrak{p}_{\alpha} \cap \mathfrak{g}^{\prime}\right)} L_{\chi, \alpha}(f)
$$

as an analogue of $Z_{\chi}(f, \alpha)$. We do not introduce extra notations for the analogues of $Z_{\chi, \alpha}(f)$ and $L_{\chi, \alpha}(f)$ because the analogous $U_{\chi}\left(\mathfrak{p}_{\alpha} \cap \mathfrak{g}^{\prime}\right)$-modules are just the restrictions of the corresponding $U_{\chi}\left(\mathfrak{p}_{\alpha}\right)$-modules.

A.6. In some cases we shall have to consider for a given $f \in \mathfrak{h}^{*}$ at the same time all $\chi$ such that $Z_{\chi}(f, \alpha)$ is defined. In that situation it will be convenient to work with a slightly modified notation.

Set

$$
\mathfrak{X}=\left\{f \in \mathfrak{h}^{*} \mid f\left(h_{\alpha}\right) \in \mathbf{F}_{p}\right\} .
$$

This is a union of $p$ affine subspaces of $\mathfrak{h}^{*}$ of codimension 1 unless $h_{\alpha}=0$ (in which case $\left.\mathfrak{X}=\mathfrak{h}^{*}\right)$. Set

$$
\Xi=\left\{\chi \in \mathfrak{g}^{*} \mid \chi\left(\mathfrak{p}_{\alpha}\right)=0\right\}
$$

Extend each $\chi(f)$ to $\mathfrak{g}$ such that $\chi(f)\left(\mathfrak{n}^{+}+\mathfrak{n}^{-}\right)=0$. Then $Z_{\chi(f)+\chi}(f, \alpha)$ is defined for all $f \in \mathfrak{X}$ and $\chi \in \Xi$. We write

$$
Z(f, \chi, \alpha)=Z_{\chi(f)+\chi}(f, \alpha) .
$$

Note also that all $Z(f+\mu, \chi, \alpha)$ with $\mu \in X$ are defined because $f+\mu \in \mathfrak{X}$.

A.7. Proposition. Let $\mu_{1}, \mu_{2} \in X$. For each integer $m$ the set

$$
\left\{(f, \chi) \in \mathfrak{X} \times \Xi \mid \operatorname{dim} \operatorname{Hom}_{\mathfrak{g}}\left(Z\left(f+\mu_{1}, \chi, \alpha\right), Z\left(f+\mu_{2}, \chi, \alpha\right)\right) \geq m\right\}
$$

is closed in $\mathfrak{X} \times \Xi$. 
Proof. The point is to show that the Hom space in (1) can be described as a space of solutions to a system of linear equations where the matrix of the system has size independent of $(f, \chi)$ and entries that are polynomial functions of $f$ and $\chi$. If we have, say, $l$ unknowns, then the Hom space has dimension $\geq m$ if and only if the rank of the matrix is $\leq l-m$ if and only if all $(l-m+1) \times(l-m+1)$ minors of the matrix are 0 . This condition clearly defines a closed subset of $\mathfrak{X} \times \Xi$.

In order to show that we are in a situation as described above, let me introduce some notation. Let $\mathfrak{R}$ be the set of all $R^{+}$-tuples of non-negative integers. To each $r=(r(\beta))_{\beta} \in \mathfrak{R}$ we associate elements

$$
x_{r}^{-}=\prod_{\beta>0} x_{-\beta}^{r(\beta)} \quad \text { and } \quad x_{r}^{+}=\prod_{\beta>0} x_{\beta}^{r(\beta)}
$$

in $U(\mathfrak{g})$ where these products are to be carried out in some fixed order. That order is arbitrary except that $x_{-\alpha}^{r(\alpha)}$ should occur in $x_{r}^{-}$as the factor most to the right.

Choose a basis $h_{1}, h_{2}, \ldots, h_{n}$ of $\mathfrak{h}$. Let $\mathfrak{S}$ be the set of all $n$-tuples of nonnegative integers. Associate to each $t=(t(i))_{i} \in \mathfrak{S}$ the element

$$
h_{t}=\prod_{i=1}^{n} h_{i}^{t(i)}
$$

in $U(\mathfrak{h}) \subset U(\mathfrak{g})$. So the

$$
x_{r}^{-} h_{t} x_{s}^{+} \quad \text { with } r, s \in \mathfrak{R}, t \in \mathfrak{S}
$$

are a PBW basis of $U(\mathfrak{g})$.

Let $d_{1}$ and $d_{2}$ be the integers with $0<d_{i} \leq p$ and $\left(f+\mu_{i}\right)\left(h_{\alpha}\right)+1=d_{i} 1$ in $\mathbf{F}_{p}$. Set $\mathfrak{R}_{i}$ equal to the set of all $r \in \mathfrak{R}$ with $r(\alpha)<d_{i}$ and $r(\beta)<p$ for all other positive roots. Then the $x_{r}^{-} v_{f+\mu_{i}}$ with $r \in \mathfrak{R}_{i}$ are a basis of $Z\left(f+\mu_{i}, \chi, \alpha\right)$.

Claim. Let $a \in \mathfrak{g}$. There exist $c_{s r}^{i}(a, f, \chi) \in K[\mathfrak{X} \times \Xi]$ such that

$$
a x_{r}^{-} v_{f+\mu_{i}}=\sum_{s \in \Re_{i}} c_{s r}^{i}(a, f, \chi) x_{s}^{-} v_{f+\mu_{i}}
$$

for all $r \in \mathfrak{R}_{i}$.

Well, to start with there are $c_{s, t, s^{\prime}, r}(a) \in K$ (independent of $f$ and $\chi$, almost all equal to 0 ) such that

$$
a x_{r}^{-}=\sum_{s \in \mathfrak{R}} \sum_{t \in \mathfrak{S}} \sum_{s^{\prime} \in \mathfrak{R}} c_{s, t, s^{\prime}, r}(a) x_{s}^{-} h_{t} x_{s^{\prime}}^{+}
$$

in $U(\mathfrak{g})$. It follows that

$$
a x_{r}^{-} v_{f+\mu_{i}}=\sum_{s \in \mathfrak{R}} \sum_{t \in \mathfrak{S}} c_{s, t, 0, r}(a)\left(f+\mu_{i}\right)\left(h_{t}\right) x_{s}^{-} v_{f+\mu_{i}} .
$$

Here 0 denotes the $R^{+}$-tuple with all components equal to 0 . Given $s \in \mathfrak{R}$ there exist unique $s^{\prime}, s^{\prime \prime} \in \mathfrak{R}$ with $s(\beta)=s^{\prime}(\beta)+p s^{\prime \prime}(\beta)$ and $0 \leq s^{\prime}(\beta)<p$ for all $\beta$. We denote then $s^{\prime}$ by $s_{\text {red }}$. Then we have

$$
x_{s}^{-} v_{f+\mu_{i}}=b_{s}(\chi) x_{s_{\mathrm{red}}}^{-} v_{f+\mu_{i}} \quad \text { with } b_{s}(\chi)=\prod_{s^{\prime \prime}(\beta)>0} \chi\left(x_{-\beta}\right)^{p s^{\prime \prime}(\beta)} .
$$


If $s_{\text {red }}(\alpha) \geq d_{i}$, then $x_{s_{\text {red }}}^{-} v_{f+\mu_{i}}=0$. So it is enough to consider in (3) only $s$ with $s_{\text {red }} \in \mathfrak{R}_{i}$. We get now a formula as in (2) with

$$
c_{s r}^{i}(a, f, \chi)=\sum_{s^{\prime} \in \mathfrak{R}, s=s_{\text {red }}^{\prime}} \sum_{t \in \mathfrak{S}} b_{s^{\prime}}(\chi) c_{s^{\prime}, t, 0, r}(a)\left(f+\mu_{i}\right)\left(h_{t}\right) .
$$

This sum is a regular function on $\mathfrak{X} \times \Xi$, since each $b_{s^{\prime}}(\chi)$ is polynomial in $\chi$ and each

$$
\left(f+\mu_{i}\right)\left(h_{t}\right)=\prod_{i=1}^{n}\left(f+\mu_{i}\right)\left(h_{i}\right)^{t(i)}
$$

is polynomial in $f$.

This finishes the proof of the claim. We now return to the proof of the proposition. The space of all linear maps from $Z\left(f+\mu_{1}, \chi, \alpha\right)$ to $Z\left(f+\mu_{2}, \chi, \alpha\right)$ has basis $E_{s r}$ with $r \in \mathfrak{R}_{1}, s \in \mathfrak{R}_{2}$ such that for all $r^{\prime} \in \mathfrak{R}_{1}$

$$
E_{s r}\left(x_{r^{\prime}}^{-} v_{f+\mu_{1}}\right)= \begin{cases}x_{s}^{-} v_{f+\mu_{2}}, & \text { if } r^{\prime}=r \\ 0, & \text { otherwise }\end{cases}
$$

Given $a \in \mathfrak{g}$ we can now use (2) to evaluate each

$$
\left(a \cdot E_{s r}\right)\left(x_{r^{\prime}}^{-} v_{f+\mu_{1}}\right)=a\left(E_{s r}\left(x_{r^{\prime}}^{-} v_{f+\mu_{1}}\right)\right)-E_{s r}\left(a x_{r^{\prime}}^{-} v_{f+\mu_{1}}\right)
$$

and get

$$
a \cdot E_{s r}=\sum_{t \in \Re_{2}} c_{t, s}^{2}(a, f, \chi) E_{t r}-\sum_{u \in \Re_{1}} c_{r, u}^{1}(a, f, \chi) E_{s u} .
$$

So we have a formula of the form

$$
a \cdot E_{s r}=\sum_{r^{\prime} \in \Re_{1}} \sum_{s^{\prime} \in \Re_{2}} b_{s^{\prime}, r^{\prime}, s, r}(a, f, \chi) E_{s^{\prime} r^{\prime}}
$$

where the $b_{s^{\prime}, r^{\prime}, s, r}(a, f, \chi)$ are regular functions of $(f, \chi) \in \mathfrak{X} \times \Xi$.

Now $\operatorname{Hom}_{\mathfrak{g}}\left(Z\left(f+\mu_{1}, \chi, \alpha\right), Z\left(f+\mu_{2}, \chi, \alpha\right)\right)$ identifies with the space of all $\left(\mathfrak{R}_{2} \times \mathfrak{R}_{1}\right)$-tuples $\left(x_{s r}\right)_{r, s}$ of elements in $K$ with $a \cdot \sum_{r, s} x_{s r} E_{s r}=0$ for all $a \in \mathfrak{g}$. It actually suffices to take for $a$ all elements in a basis (or a generating system) of $\mathfrak{g}$. This shows that we indeed get the Hom space as a solution space of a linear system of equations as described at the beginning of the proof. The proposition follows.

B.

Keep the assumptions and notations from Section A. We shall have to make in this section (and in most sections to come) two restrictive assumptions on $\mathfrak{g}$ : We assume that

$$
\text { The derived subgroup } \mathcal{D} G \text { of } G \text { is simply connected }
$$

and

The group $X / \mathbf{Z} R$ has no p-torsion.

These assumptions are needed to introduce translation functors with "nice" properties, see B.1 and B.3 below. If $G$ is semi-simple our assumptions mean that $X$ is equal to the weight lattice of $R$ and that $p$ does not divide the index of connection, i.e., the index of the root lattice $\mathbf{Z} R$ in the weight lattice. If you want to compare with the assumptions in [J3], 6.3: Our (B1) is called (H1) there, our (B2) follows from (H1) and (H3) there; see [J3], 11.2. 
B.1. Fix $\chi \in \mathfrak{g}^{*}$ with $\chi\left(\mathfrak{b}^{+}\right)=0$. The set $\Lambda_{\chi}$ from A.2(5) consists then of all $d \mu$ with $\mu \in X$. A simple $U_{\chi}(\mathfrak{g})$-module is therefore the homomorphic image of some $Z_{\chi}(\mu)$ with $\mu \in X$; cf. [J2], 1.4 or [J3], 6.7 .

The subalgebra $U(\mathfrak{g})^{G}$ (cf. [J3], 9.1) of the centre of $U(\mathfrak{g})$ acts on each $Z_{\chi}(\lambda)$ via a character. Let $\mathcal{C}_{\lambda}$ denote the category of all finite dimensional $U_{\chi}(\mathfrak{g})$-modules $M$ such that $U(\mathfrak{g})^{G}$ acts on each composition factor of $M$ via the same central character as on $Z_{\chi}(\lambda)$.

Our assumption (B1) implies that

$$
\mathcal{C}_{\lambda}=\mathcal{C}_{\mu} \Longleftrightarrow \lambda \in W \cdot \mu+p X
$$

cf. [J3], 9.4. The simple modules in $\mathcal{C}_{\lambda}$ are the simple homomorphic images of the $Z_{\chi}(w \cdot \lambda)$ with $w \in W$. Since all these $Z_{\chi}(w \bullet \lambda)$ define the same class in the Grothendieck group (cf. [J2], 1.5), we get also that the simple modules in $\mathcal{C}_{\lambda}$ are the composition factors of $Z_{\chi}(\lambda)$.

The category $\mathcal{C}$ of all finite dimensional $U_{\chi}(\mathfrak{g})$-modules is the direct sum of all $\mathcal{C}_{\lambda}$ with $\lambda$ running over representatives for the orbits on $X$ of the semi-direct product of $W$ and $p X$ [acting via $(w, p \nu) \cdot \lambda=w_{\bullet} \lambda+p \nu$ ]. Each (closed) alcove with respect to $W_{p}$ contains a representative of each orbit; cf. [J3], 11.19 or [J4], 4.1. In general, it will contain more than one representative.

If $M$ is in $\mathcal{C}$, let $\operatorname{pr}_{\lambda}(M)$ denote the largest submodule of $M$ that belongs to $\mathcal{C}_{\lambda}$. Then $M$ is the direct sum of $\operatorname{pr}_{\lambda}(M)$ and other $\operatorname{pr}_{\mu}(M)$. We get thus an exact functor $\operatorname{pr}_{\lambda}: \mathcal{C} \rightarrow \mathcal{C}_{\lambda}$.

B.2. Given $\lambda, \mu$ in a fixed (closed) alcove $C$ with respect to $W_{p}$, we define a translation functor $T_{\lambda}^{\mu}: \mathcal{C}_{\lambda} \rightarrow \mathcal{C}_{\mu}$ (as usual) by

$$
T_{\lambda}^{\mu}(V)=\operatorname{pr}_{\mu}(E \otimes V)
$$

where $E$ is the simple $G$-module with highest weight in $W(\mu-\lambda)$. (This makes sense: see the argument in [J4], 4.7 where there is a more restrictive assumption on $\chi$, which however does not affect the argument here.) We have clearly $T_{\lambda}^{\mu}=$ $T_{w \bullet \lambda+p \nu}^{w \bullet \mu+p \nu}$ for all $w \in W$ and $\nu \in X$. Note that we get (in general) more than one functor $\mathcal{C}_{\lambda} \rightarrow \mathcal{C}_{\mu}$ for fixed $\lambda$ and $\mu$ : if $\mu$ and $\mu^{\prime}$ are two distinct weights in $C$ with $\mu^{\prime} \in W \bullet \mu+p X$, then $T_{\lambda}^{\mu}$ and $T_{\lambda}^{\mu^{\prime}}$ will be two (in general:) distinct functors from $\mathcal{C}_{\lambda}$ to $\mathcal{C}_{\mu}=\mathcal{C}_{\mu^{\prime}}$.

We have as usual that each $T_{\lambda}^{\mu}$ is exact and that $T_{\lambda}^{\mu}$ and $T_{\mu}^{\lambda}$ are adjoint to each other: The natural isomorphism $\operatorname{Hom}_{\mathfrak{g}}(E \otimes M, N) \stackrel{\sim}{\longrightarrow} \operatorname{Hom}_{\mathfrak{g}}\left(M, E^{*} \otimes N\right)$ induces an isomorphism

$$
\operatorname{Hom}_{\mathfrak{g}}\left(T_{\lambda}^{\mu}(M), N\right) \stackrel{\sim}{\longrightarrow} \operatorname{Hom}_{\mathfrak{g}}\left(M, T_{\mu}^{\lambda}(N)\right)
$$

cf. [J4], 4.7 .

B.3. Let $\mu \in X$ and let $E$ be a $G$-module. The tensor identity (see [J4], 1.12(1)) says that $E \otimes Z_{\chi}(\mu)$ is isomorphic to $U_{\chi}(\mathfrak{g}) \otimes_{U_{\chi}\left(\mathfrak{b}^{+}\right)}\left(E \otimes K_{\mu}\right)$. Let $\nu_{1}, \nu_{2}, \ldots, \nu_{r}$ be the weights of $E$ (counted with multiplicities) enumerated such that $\nu_{i}>\nu_{j}$ implies $i<j$. Then $E$ considered as a $\mathfrak{b}^{+}$-module has a filtration with factors $K_{\nu_{i}}$ such that $K_{\nu_{1}}$ occurs at the bottom, then $K_{\nu_{2}}$ as the next higher factor, and so on. (To start with, we have such a filtration of $E$ considered as a module for the corresponding Borel subgroup. We then regard the terms in the filtration as $\mathfrak{b}^{+}$-modules.) It follows that $E \otimes Z_{\chi}(\mu)$ has a filtration with factors $Z_{\chi}\left(\mu+\nu_{i}\right)$ such that $Z_{\chi}\left(\mu+\nu_{1}\right)$ occurs at the bottom, then $Z_{\chi}\left(\mu+\nu_{2}\right)$ as the next higher 
factor, and so on. Each $\operatorname{pr}_{\nu}\left(E \otimes Z_{\chi}(\mu)\right)$ has then a similar filtration where only the $Z_{\chi}\left(\mu+\nu_{i}\right)$ with $\mu+\nu_{i} \in W \cdot \nu+p X$ occur. In particular, if $i_{1}$ (resp. $i_{2}$ ) is minimal (resp. maximal) among the $i$ with $\mu+\nu_{i} \in W \bullet \nu+p X$, then $Z_{\chi}\left(\mu+\nu_{i_{1}}\right)$ is a submodule of $\operatorname{pr}_{\nu}\left(E \otimes Z_{\chi}(\mu)\right)$, and $Z_{\chi}\left(\mu+\nu_{i_{2}}\right)$ is a homomorphic image of $\operatorname{pr}_{\nu}\left(E \otimes Z_{\chi}(\mu)\right)$.

For the following result we have to use the assumption (B2) made at the beginning of this section.

Proposition. Let $\lambda, \mu \in X$ and $w \in W_{p}$. Suppose that there exists a closed alcove with respect to $W_{p}$ containing $\lambda$ and $\mu$. Then $T_{\lambda}^{\mu} Z_{\chi}\left(w_{\bullet} \lambda\right)$ has a filtration with factors $Z_{\chi}\left(w w_{1} \bullet \mu\right)$ with $w_{1} \in W_{p}, w_{1} \bullet \lambda=\lambda$. There is one factor for each weight of the form $w w_{1} \bullet \mu$.

Proof. Consider $E$ as in the definition of $T_{\lambda}^{\mu}$. The discussion above shows that $T_{\lambda}^{\mu} Z_{\chi}(w \cdot \lambda)$ has a filtration with factors $Z_{\chi}(w \cdot \lambda+\nu)$ where $\nu$ runs over all weights of $E$ with $w_{\bullet} \lambda+\nu \in W \bullet \mu+p X$. Suppose that $\nu$ has this property; so there are $w_{1} \in W$ and $\nu_{1} \in X$ with $w_{\bullet} \lambda+\nu=w_{1} \bullet \mu+p \nu_{1}$. We have $w_{\bullet} \lambda \in \lambda+\mathbf{Z} R$ and $\nu \in(\mu-\lambda)+\mathbf{Z} R$ and $w_{1} \bullet \mu \in \mu+\mathbf{Z} R$, hence $p \nu_{1} \in \mathbf{Z} R$. In other words, we have $p\left(\nu_{1}+\mathbf{Z} R\right)=0$ in $X / \mathbf{Z} R$. Assumption (B2) yields now $\nu_{1} \in \mathbf{Z} R$, hence $w_{1} \bullet \mu+p \nu_{1} \in W_{p} \bullet \mu$. So $T_{\lambda}^{\mu} Z_{\chi}\left(w_{\bullet} \lambda\right)$ has a filtration with factors $Z_{\chi}\left(w_{\bullet} \lambda+\nu\right)$ where $\nu$ runs over those weights of $E$ with $w_{\bullet} \lambda+p \nu \in W_{p} \bullet \mu+p X$. Now the claim follows from standard results; cf. [J1], II.7.13.

B.4. Note that Proposition B.3 implies in particular: If $\mu$ is in the closure of the facet of $\lambda$ with respect to $W_{p}$, then we have

$$
T_{\lambda}^{\mu} Z_{\chi}(w \bullet \lambda) \simeq Z_{\chi}(w \bullet \mu)
$$

for all $w \in W_{p}$.

Lemma. If $\mu$ is in the closure of the facet of $\lambda$ with respect to $W_{p}$, then we have $T_{\mu}^{\lambda} L \neq 0$ for all simple modules $L$ in $\mathcal{C}_{\mu}$.

Proof. There exists $w \in W$ with $\operatorname{Hom}_{\mathfrak{g}}\left(Z_{\chi}(w \bullet \mu), L\right) \neq 0$. Now (1) and the adjointness property B.2(2) imply that

$$
\operatorname{Hom}_{\mathfrak{g}}\left(Z_{\chi}(w \bullet \lambda), T_{\mu}^{\lambda} L\right) \simeq \operatorname{Hom}_{\mathfrak{g}}\left(Z_{\chi}(w \bullet \mu), L\right) \neq 0,
$$

hence the claim.

Remark. The claim extends of course to all non-zero modules in $\mathcal{C}_{\mu}$.

B.5. Proposition. Suppose that $\lambda$ and $\mu$ belong to the same facet with respect to $W_{p}$. Then $T_{\lambda}^{\mu} L$ is simple for each simple module $L$ in $\mathcal{C}_{\lambda}$. The functor $T_{\lambda}^{\mu}$ induces a bijection from the isomorphism classes of simple modules in $\mathcal{C}_{\lambda}$ to the isomorphism classes of simple modules in $\mathcal{C}_{\mu}$; the inverse is induced by $T_{\mu}^{\lambda}$.

Proof. Consider a composition series

$$
Z_{\chi}(\lambda)=M_{r} \supset M_{r-1} \supset \cdots \supset M_{1} \supset M_{0}=0
$$

of $Z_{\chi}(\lambda)$. The exactness of $T_{\lambda}^{\mu}$ and B.4(1) yield a chain of submodules

$$
Z_{\chi}(\mu) \simeq T_{\lambda}^{\mu} M_{r} \supset T_{\lambda}^{\mu} M_{r-1} \supset \cdots \supset T_{\lambda}^{\mu} M_{1} \supset T_{\lambda}^{\mu} M_{0}=0 .
$$

Each $T_{\lambda}^{\mu} M_{i} / T_{\lambda}^{\mu} M_{i-1} \simeq T_{\lambda}^{\mu}\left(M_{i} / M_{i-1}\right)$ is non-zero by Lemma B.4. It follows that the length of $Z_{\chi}(\mu)$ is greater than or equal to the length of $Z_{\chi}(\lambda)$. By symmetry we 
get also the reversed inequality. Therefore both modules have the same length. This implies that all $T_{\lambda}^{\mu}\left(M_{i} / M_{i+1}\right)$ are simple, hence the first claim of the proposition.

We get by symmetry: If $L^{\prime}$ is simple in $\mathcal{C}_{\mu}$, then also $T_{\mu}^{\lambda} L^{\prime}$ is simple. It follows that $T_{\mu}^{\lambda} T_{\lambda}^{\mu} L$ is simple for all $L$ simple in $\mathcal{C}_{\lambda}$. We have by adjointness

$$
\operatorname{Hom}_{\mathfrak{g}}\left(T_{\mu}^{\lambda} T_{\lambda}^{\mu} L, L\right) \simeq \operatorname{Hom}_{\mathfrak{g}}\left(T_{\lambda}^{\mu} L, T_{\lambda}^{\mu} L\right) \neq 0,
$$

hence $T_{\mu}^{\lambda} T_{\lambda}^{\mu} L \simeq L$ (both modules being simple). We get by symmetry: $T_{\lambda}^{\mu} T_{\mu}^{\lambda} L^{\prime} \simeq$ $L^{\prime}$ for all $L^{\prime}$ simple in $\mathcal{C}_{\mu}$. The second claim follows.

B.6. Let $I$ be a subset of the set of simple roots. Let $P_{I}$ be the corresponding parabolic subgroup of $G$ and $\mathfrak{p}_{I}$ its Lie algebra. So $\mathfrak{p}_{I}$ is the direct sum of $\mathfrak{b}^{+}$and the $\mathfrak{g}_{\beta}$ with $\beta<0$ and $\beta \in R_{I}=R \cap \mathbf{Z I}$. Let $G_{I} \supset T$ be the standard Levi factor of $P_{I}$ and $\mathfrak{g}_{I}$ its Lie algebra, $\mathfrak{g}_{I}=\mathfrak{h} \oplus \bigoplus_{\beta \in R_{I}} \mathfrak{g}_{\beta}$.

For each $\lambda \in X$ set

$$
Z_{\chi, I}(\lambda)=U_{\chi}\left(\mathfrak{p}_{I}\right) \otimes_{U_{\chi}\left(\mathfrak{b}^{+}\right)} K_{\lambda} .
$$

Note that this generalises the case $|I|=1$ considered in A.3(1) except for that we consider here more restrictive $\chi$.

We have [as in A.4(1)] by transitivity of induction an isomorphism of $U_{\chi}(\mathfrak{g})$ modules

$$
Z_{\chi}(\lambda) \stackrel{\sim}{\longrightarrow} U_{\chi}(\mathfrak{g}) \otimes_{U_{\chi}\left(\mathfrak{p}_{I}\right)} Z_{\chi, I}(\lambda) .
$$

If we write $v_{\lambda, I}$ for the standard generator $1 \otimes 1$ of $Z_{\chi, I}(\lambda)$, then the isomorphism in (2) takes $v_{\lambda}$ to $1 \otimes v_{\lambda, I}$. The nilradical $\mathfrak{n}^{I}$ of $\mathfrak{p}_{I}$ (the sum of the $\mathfrak{g}_{\alpha}$ with $\alpha>0$ and $\alpha \notin R_{I}$ ) annihilates each $Z_{\chi, I}(\lambda)$. Considered as a module over $\mathfrak{g}_{I} \simeq \mathfrak{p}_{I} / \mathfrak{n}^{I}$, each $Z_{\chi, I}(\lambda)$ identifies with the corresponding baby Verma module for $U_{\chi}\left(\mathfrak{g}_{I}\right)$.

The Weyl group $W_{I}$ of $R_{I}$ identifies with the subgroup of $W$ generated by the $s_{\alpha}$ with $\alpha \in I$; similarly for the corresponding affine Weyl group $W_{I, p}$. The group $G_{I}$ again satisfies (B1) and (B2); for (B2) note that $\mathbf{Z} R_{I}=\mathbf{Z} I$ is a direct summand of $\mathbf{Z} R$. We define categories $\mathcal{C}(I)_{\lambda}$ of finite dimensional $U_{\chi}\left(\mathfrak{g}_{I}\right)$-modules analogous to the $\mathcal{C}_{\lambda}$ : A finite dimensional $U_{\chi}\left(\mathfrak{g}_{I}\right)$-module $M$ belongs to $\mathcal{C}(I)_{\lambda}$ if and only if all its composition factors are composition factors of some $Z_{\chi, I}(w \bullet \lambda)$ with $w \in W_{I}$.

We can extend each $U_{\chi}\left(\mathfrak{g}_{I}\right)$-module $M$ to a $U_{\chi}\left(\mathfrak{p}_{I}\right)$-module letting $\mathfrak{n}^{I}$ act trivially. We get then an induced $U_{\chi}(\mathfrak{g})$-module that we shall denote by $\operatorname{ind}_{I} M$ :

$$
\operatorname{ind}_{I} M=U_{\chi}(\mathfrak{g}) \otimes_{U_{\chi}\left(\mathfrak{p}_{I}\right)} M .
$$

We have by (2) and the remarks following it

$$
\operatorname{ind}_{I} Z_{\chi, I}(\lambda) \simeq Z_{\chi}(\lambda) .
$$

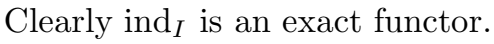

Lemma. Let $\lambda \in X$. If $M$ is in $\mathcal{C}(I)_{\lambda}$, then $\operatorname{ind}_{I}(M)$ is in $\mathcal{C}_{\lambda}$.

Proof. This is clear for $M=Z_{\chi, I}\left(w_{\bullet} \lambda\right)$ with $w \in W_{I}$ by (4). It then follows (by the exactness of ind $I$ ) first for all simple modules in $\mathcal{C}(I)_{\lambda}$ and then for all $M$ in $\mathcal{C}(I)_{\lambda}$. 
B.7. Keep the notations and assumptions from B.6. The category of all finite dimensional $U_{\chi}\left(\mathfrak{g}_{I}\right)$-modules is the direct sum of all $\mathcal{C}(I)_{\lambda}$ with $\lambda$ running over suitable representatives. We denote the corresponding projection functors by $\operatorname{pr}(I)_{\lambda}$.

If $\lambda$ and $\mu$ are weights in the same (closed) alcove with respect to $W_{I, p}$, then we can define a translation functor $T(I)_{\lambda}^{\mu}$ working with the simple $G_{I}$-module $E^{\prime}$ with highest weight in $W_{I}(\mu-\lambda)$.

If $\lambda$ and $\mu$ are weights in the same (closed) alcove with respect to $W_{p}$, then $\lambda$ and $\mu$ belong also to the same (closed) alcove with respect to $W_{I, p}$. So both $T_{\lambda}^{\mu}$ and $T(I)_{\lambda}^{\mu}$ are defined and we want to compare them. Choose $w_{1}, w_{2}, \ldots, w_{r} \in W_{p}$ with $w_{i} \bullet \lambda=\lambda$ such that each $w_{\bullet} \mu$ with $w \in \operatorname{Stab}_{W_{p}} \lambda$ is conjugate to exactly one $w_{i} \bullet \mu$ under the stabiliser of $\lambda$ in $W_{I, p}$.

Proposition. There exists for each $V$ in $\mathcal{C}(I)_{\lambda}$ a filtration of $T_{\lambda}^{\mu}\left(\operatorname{ind}_{I} V\right)$ with factors isomorphic to $\operatorname{ind}_{I}\left(T(I)_{\lambda}^{w_{i} \bullet \mu} V\right), 1 \leq i \leq r$.

Proof. [Note that we do not claim that the factors in the filtration occur in the same order as the indices.]

Let $E$ be the simple $G$-module with highest weight in $W(\mu-\lambda)$. The tensor identity yields an isomorphism

$$
E \otimes \operatorname{ind}_{I} V=E \otimes\left(U_{\chi} \otimes_{U_{\chi}(\mathfrak{g})\left(\mathfrak{p}_{I}\right)} V\right) \stackrel{\sim}{\longrightarrow} U_{\chi}(\mathfrak{g}) \otimes_{U_{\chi}\left(\mathfrak{p}_{I}\right)}(E \otimes V)
$$

that takes any $e \otimes(1 \otimes v)$ with $e \in E$ and $v \in V$ to $1 \otimes(e \otimes v)$. We get thus a functorial isomorphism

$$
T_{\lambda}^{\mu}\left(\operatorname{ind}_{I} V\right) \stackrel{\sim}{\longrightarrow} \operatorname{pr}_{\mu} U_{\chi}(\mathfrak{g}) \otimes_{U_{\chi}\left(\mathfrak{p}_{I}\right)}(E \otimes V) .
$$

Consider a composition series of $E$ as a $P_{I^{-}}$-module. The unipotent radical of $P_{I}$ acts trivially on the factors (hence so does its Lie algebra $\mathfrak{n}^{I}$ ) and these factors are simple $G_{I}$-modules. It follows that $T_{\lambda}^{\mu}\left(\operatorname{ind}_{I} V\right)$ has a filtration with factors $\operatorname{pr}_{\mu} \operatorname{ind}_{I}(L \otimes V)$ with $L$ running over the factors in a composition series of $E$ as a $P_{I}$-module. Each $L \otimes V$ is the direct sum of all $\operatorname{pr}(I)_{\mu^{\prime}}(L \otimes V)$ with $\mu^{\prime}$ running over representatives for the orbits of $W_{I}$ on $X / p X$. So $T_{\lambda}^{\mu}\left(\operatorname{ind}_{I} V\right)$ has a filtration with factors

$$
\operatorname{pr}_{\mu} \operatorname{ind}_{I} \operatorname{pr}(I)_{\mu^{\prime}}(L \otimes V)
$$

with $L$ and $\mu^{\prime}$ as above.

Since $\mu-\lambda$ is a weight of $E$ with multiplicity 1 , so is each $w_{i} \bullet \mu-\lambda=w_{i} \bullet \mu-w_{i} \bullet \lambda \in$ $W(\mu-\lambda)$. Therefore our composition series of $E$ as a $P_{I}$-module contains exactly one factor, say $E_{i}$, such that $w_{i} \bullet \mu-\lambda$ is a weight of $E_{i}$. Now $w_{i} \bullet \mu-\lambda$ is an extremal weight of the $G_{I}$-module $E_{i}$ since it is an extremal weight of the $G$-module $E$. (The last statement means for all $\beta \in R$ that either no $w_{i} \bullet \mu-\lambda+n \beta$ with $n>0$ is a weight of $E$ or that no $w_{i} \bullet \mu-\lambda-n \beta$ with $n>0$ is a weight of $E$ or both.) Therefore $w_{i} \bullet \mu-\lambda$ is conjugate to the highest weight of $E_{i}$ under $W_{I}$ and we can take $E_{i}$ as the simple module $E^{\prime}$ used in the definition of $T(I)_{\lambda}^{w_{i} \bullet \mu}$. This means that

$$
T(I)_{\lambda}^{w_{i} \bullet \mu}(V) \simeq \operatorname{pr}(I)_{w_{i} \bullet \mu}\left(E_{i} \otimes V\right) .
$$

So $\operatorname{ind}_{I}\left(T(I)_{\lambda}^{w_{i} \bullet \mu} V\right)$ is one of the factors as in (2). [Note that $\operatorname{ind}_{I}\left(T(I)_{\lambda}^{w_{i} \bullet \mu} V\right)$ $=\operatorname{pr}_{\mu} \operatorname{ind}_{I}\left(T(I)_{\lambda}^{w_{i} \bullet \mu} V\right)$ by Lemma B.6.]

Let us check next that distinct $i$ lead here to distinct factors. Otherwise we have $i \neq j$ with $\left(E_{i}, \operatorname{pr}(I)_{w_{i} \bullet \mu}\right)=\left(E_{j}, \operatorname{pr}(I)_{w_{j} \bullet \mu}\right)$. This implies $w_{i} \bullet \mu-\lambda \in W_{I}\left(w_{j} \bullet \mu-\lambda\right)$ and $w_{i} \bullet \mu \in W_{I} \bullet\left(w_{j} \bullet \mu\right)+p X$. The first property yields $w_{i} \bullet \mu \in w_{j} \bullet \mu+\mathbf{Z} I$; since 
both $X / \mathbf{Z} R$ and $\mathbf{Z} R / \mathbf{Z} I$ have no $p$-torsion, the second property implies that $w_{i} \bullet \mu \in$ $W_{I} \bullet\left(w_{j} \bullet \mu\right)+p \mathbf{Z} I=W_{I, p} \bullet\left(w_{j} \bullet \mu\right)$. So $\nu=w_{i} \bullet \mu-\lambda$ is a weight of the simple $G_{I^{-}}$ module with extremal weight $w_{j} \bullet \mu-\lambda$ such that $\lambda+\nu \in W_{I} \bullet\left(w_{j} \bullet \mu\right)$. Now [J1], II.7.7 implies that there exists $w \in W_{I, p}$ with $w_{\bullet} \lambda=\lambda$ and $w_{\bullet}\left(w_{j} \bullet \mu\right)=\lambda+\nu=w_{i} \bullet \mu$. This is a contradiction to the choice of the $w_{i}$.

We have so far seen that all $\operatorname{ind}_{I}\left(T(I)_{\lambda}^{w_{i} \bullet \mu} V\right)$ occur in the filtration of $T_{\lambda}^{\mu}\left(\operatorname{ind}_{I} V\right)$ with factors as in (2). It remains to be shown that all remaining factors as in (2) are 0 . Using the exactness of $\operatorname{pr}_{\mu}$, of all $\operatorname{pr}(I)_{\mu^{\prime}}$, and of the induction one reduces first to the case where $V$ is simple and then to the case where $V=Z_{\chi, I}(w \bullet \lambda)$ for some $w \in W_{I}$. That all other factors as in (2) are 0 in this case will follow if we can show that

$$
\operatorname{dim} T_{\lambda}^{\mu}\left(\operatorname{ind}_{I} V\right)=\sum_{i=1}^{r} \operatorname{dim} \operatorname{ind}_{I}\left(T(I)_{\lambda}^{w_{i} \bullet \mu} V\right)
$$

for $V=Z_{\chi, I}(w \cdot \lambda)$. In this case $\operatorname{ind}_{I} V \simeq Z_{\chi}(w \bullet \lambda)$; so $T_{\lambda}^{\mu}\left(\operatorname{ind}_{I} V\right)$ has by B.3 a filtration with factors $Z_{\chi}\left(w^{\prime} \bullet \mu\right)$ with $w^{\prime} \bullet \mu$ running over $w \operatorname{Stab}_{W_{p}}(\lambda) \bullet \mu$. Similarly, each $T(I)_{\lambda}^{w_{i} \bullet \mu} V$ has a filtration with factors $Z_{\chi, I}\left(w^{\prime} w_{i} \bullet \mu\right)$ with $w^{\prime} w_{i} \bullet \mu$ running over $w \operatorname{Stab}_{W_{I, p}}(\lambda) w_{i} \bullet \mu$. Then $\operatorname{ind}_{I}\left(T(I)_{\lambda}^{w_{i} \bullet \mu} V\right)$ has a filtration with factors $Z_{\chi}\left(w^{\prime} w_{i} \bullet \mu\right)$ and $w^{\prime}$ as before. Now the claim follows because $\operatorname{Stab}_{W_{p}}(\lambda) \bullet \mu$ is the disjoint union of the $\operatorname{Stab}_{W_{I, p}}(\lambda) w_{i} \bullet \mu$.

Remark. The following situation is particularly easy: Suppose that $\mu$ is contained in the closure of the facet of $\lambda$ with respect to $W_{p}$. Then there is only one factor in the filtration (since $w \bullet \mu=\mu$ for all $w$ in the stabiliser of $\lambda$ ). So we get in this case an isomorphism

$$
T_{\lambda}^{\mu}\left(\operatorname{ind}_{I} V\right) \stackrel{\sim}{\longrightarrow} \operatorname{ind}_{I}\left(T(I)_{\lambda}^{\mu} V\right) .
$$

This map is functorial: We have for each morphism $\varphi: V \rightarrow V^{\prime}$ in $\mathcal{C}(I)_{\lambda}$ a commutative diagram

$$
\begin{array}{rlr}
T_{\lambda}^{\mu}\left(\operatorname{ind}_{I} V\right) & \stackrel{\sim}{\longrightarrow} \operatorname{ind}_{I}\left(T(I)_{\lambda}^{\mu} V\right) \\
T_{\lambda}^{\mu}\left(\operatorname{ind}_{I} \varphi\right) \downarrow & \downarrow \operatorname{ind}_{I}\left(T(I)_{\lambda}^{\mu}(\varphi)\right) \\
T_{\lambda}^{\mu}\left(\operatorname{ind}_{I} V^{\prime}\right) & \stackrel{\sim}{\longrightarrow} \operatorname{ind}_{I}\left(T(I)_{\lambda}^{\mu} V^{\prime}\right)
\end{array}
$$

where the horizontal maps are isomorphisms as in (3). This follows from the functoriality of the isomorphism in (1) and the fact that $\operatorname{id}_{E} \otimes \varphi$ induces morphisms $\operatorname{pr}(I)_{\mu^{\prime}}(L \otimes V) \rightarrow \operatorname{pr}(I)_{\mu^{\prime}}\left(L \otimes V^{\prime}\right)$ for all $L$ and $\mu^{\prime}$ as in (2).

B.8. We want to apply B.6 and B.7 in the case where $|I|=1$. We first have to prove some results on $T(I)_{\lambda}^{\mu}$ in that case. In order to simplify the notations we assume in this and the following subsection that $\mathfrak{g}_{I}=\mathfrak{g}$.

So assume now that $G$ has semi-simple rank equal to 1 . There is then exactly one positive root; denote it by $\alpha$. In this case our baby Verma module $Z_{\chi}(\lambda)$ coincides with the module $Z_{\chi, \alpha}(\lambda)$ in the notation from A.3. We can use the explicit description of a basis for this module there, and we have the homomorphism $\varphi_{\lambda}: Z_{\chi}(\lambda-d \alpha) \rightarrow Z_{\chi}(\lambda)$ as in A.3(3) given by $\varphi_{\lambda}\left(v_{\lambda-d \alpha}\right)=x_{-\alpha}^{d} v_{\lambda}$. Here $d$ is the integer with $0<d \leq p$ and $\left\langle\lambda+\rho, \alpha^{\vee}\right\rangle \equiv d(\bmod p)$, hence $\lambda\left(h_{\alpha}\right)+1=d 1$ in $\mathbf{F}_{p}$. We claim now that

$$
\operatorname{Hom}_{\mathfrak{g}}\left(Z_{\chi}(\lambda-d \alpha), Z_{\chi}(\lambda)\right)=K \varphi_{\lambda} \quad \text { if } d<p .
$$


Indeed, our assumption (B2) implies that $\lambda-i \alpha \not \equiv \lambda-j \alpha(\bmod p X)$ whenever $0 \leq$ $i, j<p$ and $i \neq j$. So the $x_{-\alpha}^{i} v_{\lambda}$ with $0 \leq i<p$ belong to distinct eigenspaces with respect to $\mathfrak{h}$, hence span these eigenspaces. Each homomorphism from $Z_{\chi}(\lambda-d \alpha)$ to $Z_{\chi}(\lambda)$ has to take $\varphi\left(v_{\lambda-d \alpha}\right)$ to an eigenvector for the same eigenvalue, hence to a multiple of $x_{-\alpha}^{d} v_{\lambda}$. So (1) follows.

The same argument shows that

$$
\operatorname{Hom}_{\mathfrak{g}}\left(Z_{\chi}(\lambda-p \alpha), Z_{\chi}(\lambda)\right)=\operatorname{Hom}_{\mathfrak{g}}\left(Z_{\chi}(\lambda), Z_{\chi}(\lambda)\right)=K \text { id } .
$$

(Note that $Z_{\chi}(\lambda-p \alpha)=Z_{\chi}(\lambda)$.) If $d=p$, then $\varphi_{\lambda}$ is equal to multiplication by $\chi\left(x_{-\alpha}\right)^{p}$ since $x_{-\alpha}^{[p]}=0$. Therefore (1) extends to the case $d=p$ if $\chi\left(x_{-\alpha}\right) \neq 0$. For $\chi\left(x_{-\alpha}\right)=0$, however, we have $\varphi_{\lambda}=0$ in this case.

B.9. Keep the assumptions from B.8. Consider now two weights $\lambda$ and $\mu$ such that $\mu$ is in the closure of the facet of $\lambda$ with respect to $W_{p}$. There is a unique integer $n$ with

$$
n p<\left\langle\lambda+\rho, \alpha^{\vee}\right\rangle \leq(n+1) p .
$$

The assumption on the facet implies that

$$
n p \leq\left\langle\mu+\rho, \alpha^{\vee}\right\rangle \leq(n+1) p .
$$

Set $d=\left\langle\lambda+\rho, \alpha^{\vee}\right\rangle-n p$ and $d^{\prime}=\left\langle\mu+\rho, \alpha^{\vee}\right\rangle-n p$. Then $d$ is the same number as considered in B.8. If $d^{\prime}>0$, then it is the analogue of $d$ working with $\mu$ instead of $\lambda$; if $d^{\prime}=0$, then that analogue is equal to $p$, however. Note that $s_{\alpha, n p} \bullet \lambda=\lambda-d \alpha$ and $s_{\alpha, n p} \bullet \mu=\mu-d^{\prime} \alpha$.

We get from B.4(1) isomorphisms $T_{\lambda}^{\mu} Z_{\chi}(\lambda) \simeq Z_{\chi}(\mu)$ and $T_{\lambda}^{\mu} Z_{\chi}(\lambda-d \alpha) \simeq$ $Z_{\chi}\left(\mu-d^{\prime} \alpha\right)$. We claim that, modulo these isomorphisms:

Lemma. If $d^{\prime}>0$, then $T_{\lambda}^{\mu}\left(\varphi_{\lambda}\right)$ is a non-zero multiple of $\varphi_{\mu}$. If $d^{\prime}=0$, then $T_{\lambda}^{\mu}\left(\varphi_{\lambda}\right)$ is a non-zero multiple of the identity.

Proof. If $d=p$, i.e., if $\left\langle\lambda+\rho, \alpha^{\vee}\right\rangle=(n+1) p$, then the assumption on the facets implies that also $\left\langle\mu+\rho, \alpha^{\vee}\right\rangle=(n+1) p$ and $d^{\prime}=p$. In this case both $\varphi_{\lambda}$ and $\varphi_{\mu}$ are equal to $\chi\left(x_{-\alpha}\right)^{p}$ times the identity. Since the functor $T_{\lambda}^{\mu}$ takes a multiple of the identity to the corresponding multiple of the identity, the claim follows in this case.

Assume from now on that $d<p$.

Case I: We have $\left\langle\mu+\rho, \alpha^{\vee}\right\rangle \leq\left\langle\lambda+\rho, \alpha^{\vee}\right\rangle$. Then the module $E$ involved in the construction of $T_{\lambda}^{\mu}$ has highest weight $s_{\alpha}(\mu-\lambda)=\mu-\lambda+\left(d-d^{\prime}\right) \alpha$. If $e_{0}$ is a highest weight vector of $E$, then $v=e_{0} \otimes v_{\lambda-d \alpha}$ is a highest weight vector of weight $\mu-d^{\prime} \alpha$ in $E \otimes Z_{\chi}(\lambda-d \alpha)$ and generates the summand $T_{\lambda}^{\mu} Z_{\chi}(\lambda-d \alpha) \simeq Z_{\chi}(\mu-d \alpha)$.

The map $T_{\lambda}^{\mu}\left(\varphi_{\lambda}\right)$ is the restriction of $\operatorname{id}_{E} \otimes \varphi_{\lambda}$ to $T_{\lambda}^{\mu} Z_{\chi}(\lambda-d \alpha)$. It maps $v$ to $e_{0} \otimes x_{-\alpha}^{d} v_{\lambda}$, hence is non-zero (since $d<p$ ). Now the claim follows from B.8(1) in case $d^{\prime}>0$, or from B.8(2) in case $d^{\prime}=0$.

Case II: We have $\left.\left\langle\mu+\rho, \alpha^{\vee}\right\rangle\right\rangle\left\langle\lambda+\rho, \alpha^{\vee}\right\rangle$. Now $E$ has highest weight $\mu-\lambda$. It has a basis $e_{i}=x_{-\alpha}^{i} e_{0}$ with $0 \leq i<d^{\prime}-d=\left\langle\mu-\lambda, \alpha^{\vee}\right\rangle$ where $e_{0}$ is a weight vector of weight $\mu-\lambda$ and satisfies $x_{\alpha} e_{0}=0$. We have $x_{\alpha} e_{i}=i\left(d^{\prime}-d+1-i\right) e_{i-1}$ for all $i>0$. A straightforward calculation shows that $T_{\lambda}^{\mu} Z_{\chi}(\lambda-d \alpha) \simeq Z_{\chi}\left(\mu-d^{\prime} \alpha\right)$ is generated by

$$
v=\sum_{i=0}^{d^{\prime}-d}\left(\begin{array}{c}
d^{\prime} \\
i
\end{array}\right) e_{i} \otimes x_{-\alpha}^{d^{\prime}-d-i} v_{\lambda-d \alpha} .
$$


(Note that $v \neq 0$ since the coefficient of $e_{0} \otimes x_{-\alpha}^{d^{\prime}-d} v_{\lambda-d \alpha}$ is non-zero.) We have now

$$
T_{\lambda}^{\mu}\left(\varphi_{\lambda}\right) v=\sum_{i=0}^{d^{\prime}-d}\left(\begin{array}{c}
d^{\prime} \\
i
\end{array}\right) e_{i} \otimes x_{-\alpha}^{d^{\prime}-i} v_{\lambda-d \alpha} .
$$

If $d^{\prime}<p$, then a look at the "leading term" $e_{0} \otimes x_{-\alpha}^{d^{\prime}} v_{\lambda-d \alpha}$ shows that $T_{\lambda}^{\mu}\left(\varphi_{\lambda}\right) \neq 0$ and the claim follows from B.8(1). If $d^{\prime}=p$, then

$$
T_{\lambda}^{\mu}\left(\varphi_{\lambda}\right) v=e_{0} \otimes x_{-\alpha}^{p} v_{\lambda-d \alpha}=\chi\left(x_{-\alpha}\right)^{p} e_{0} \otimes v_{\lambda-d \alpha} .
$$

We see thus that $T_{\lambda}^{\mu}\left(\varphi_{\lambda}\right) \neq 0$ if and only if $\chi\left(x_{-\alpha}\right) \neq 0$ if and only if $\varphi_{\mu} \neq 0$. Now the claim is obvious in case $\chi\left(x_{-\alpha}\right)=0$ and follows otherwise from B.8(2).

B.10. Let $G$ again be arbitrary. Choose a simple root $\alpha$ and set $I=\{\alpha\}$. We get from A.4(2) for each $\lambda \in X$ a homomorphism $\varphi_{\alpha}^{\lambda}: Z_{\chi}(\lambda-d \alpha) \rightarrow Z_{\chi}(\lambda)$ with $\varphi_{\alpha}^{\lambda}\left(v_{\lambda-d \alpha}\right)=x_{-\alpha}^{d} v_{\lambda}$. Here $d$ is again the integer with $0<d \leq p$ and $\left\langle\lambda+\rho, \alpha^{\vee}\right\rangle \equiv d$ $(\bmod p)$.

We can identify $Z_{\chi}(\lambda)$ with $\operatorname{ind}_{I} Z_{\chi, \alpha}(\lambda)$ [see A.4(1)]; then $\varphi_{\alpha}^{\lambda}$ identifies with $\operatorname{ind}_{I}\left(\varphi_{\lambda}\right)$.

Proposition. Let $\lambda, \mu \in X$ such that $\mu$ is contained in the closure of the facet of $\lambda$ with respect to $W_{p}$. If $\left\langle\mu+\rho, \alpha^{\vee}\right\rangle \equiv 0(\bmod p)$ and $\left\langle\mu+\rho, \alpha^{\vee}\right\rangle\left\langle\left\langle\lambda+\rho, \alpha^{\vee}\right\rangle\right.$, then $T_{\lambda}^{\mu}\left(\varphi_{\alpha}^{\lambda}\right)$ identifies with a non-zero multiple of the identity on $Z_{\chi}(\mu)$. Otherwise $T_{\lambda}^{\mu}\left(\varphi_{\alpha}^{\lambda}\right)$ identifies with a non-zero multiple of $\varphi_{\alpha}^{\mu}$.

Proof. As stated above, we can identify $\varphi_{\alpha}^{\lambda}$ with $\operatorname{ind}_{I}\left(\varphi_{\lambda}\right)$. The functoriality in B.7(3) implies that we can identify $T_{\lambda}^{\mu}\left(\varphi_{\alpha}^{\lambda}\right)$ with $\operatorname{ind}_{I}\left(T(I)_{\lambda}^{\mu} \varphi_{\lambda}\right)$. Now the claim follows from Lemma B.9 applied to $\mathfrak{g}_{I}$. (Note that the first case in the present proposition corresponds to the case $d^{\prime}=0$ in B.9.)

B.11. Let $\alpha$ be a simple root with $\chi\left(x_{-\alpha}\right)=0$. In this situation we have defined in A.4 the module $Z_{\chi}(\lambda, \alpha)$ as the cokernel of $\varphi_{\alpha}^{\lambda}$.

Corollary. Let $\lambda, \mu \in X$ such that $\mu$ is contained in the closure of the facet of $\lambda$ with respect to $W_{p}$. If $\left\langle\mu+\rho, \alpha^{\vee}\right\rangle \equiv 0(\bmod p)$ and $\left\langle\mu+\rho, \alpha^{\vee}\right\rangle\left\langle\left\langle\lambda+\rho, \alpha^{\vee}\right\rangle\right.$, then $T_{\lambda}^{\mu} Z_{\chi}(\lambda, \alpha)=0$. Otherwise $T_{\lambda}^{\mu} Z_{\chi}(\lambda, \alpha) \simeq Z_{\chi}(\mu, \alpha)$.

Proof. This follows from Proposition B.10 when we apply the exact functor $T_{\lambda}^{\mu}$ to the exact sequence A.4(3).

Remark. Let $I$ be a subset of the set of simple roots such that the restriction of $\chi$ to $\mathfrak{g}_{I}$ has standard Levi form (see [J3], 10.1 or [J4], 2.2). We have then for each $\lambda \in X$ a unique simple quotient $L_{\chi, I}(\lambda)$ of $Z_{\chi, I}(\lambda)$ and can define induced modules $Z_{\chi}(\lambda, I)=\operatorname{ind}_{I} L_{\chi, I}(\lambda)$. We can apply B.7(3) and describe $T_{\lambda}^{\mu} Z_{\chi}(\lambda, I)$ (for $\mu$ in the closure of the facet of $\lambda$ ) using [J4], 4.11(4). The corollary above is a special case of that more general result.

B.12. We shall always write $[M: L]$ to denote the multiplicity of a simple module $L$ as a composition factor of a module $M$.

For each simple $U_{\chi}(\mathfrak{g})$-module $L$ let $Q_{L}$ denote the projective cover of $L$ in the category of all $U_{\chi}(\mathfrak{g})$-modules.

The proof of Lemma 10.9 in [J3] shows that

$$
\operatorname{dim} Q_{L}=p^{N} \sum_{\mu}\left[Z_{\chi}(\mu): L\right]
$$


where $N=\left|R^{+}\right|$and where $\mu$ runs over a system of representatives for $X / p X$. (In [J3], 10.9 one has a more restrictive assumption on $\chi$ that actually does not enter the argument.)

Let $\lambda \in X$ and let $L$ be a simple module in $\mathcal{C}_{\lambda}$. Then only $\mu \in W \cdot \lambda+p X$ can contribute a positive multiplicity $\left[Z_{\chi}(\mu): L\right]$. Since all $Z_{\chi}(\mu)$ with $\mu \in W \cdot \lambda+p X$ define the same class in the Grothendieck group (cf. [J2], 1.5), we get from (1) that

$$
\operatorname{dim} Q_{L}=p^{N}|W \cdot(\lambda+p X)|\left[Z_{\chi}(\lambda): L\right] .
$$

Remark. Our assumptions (B1) and (B2) are not needed to prove (1). For (2) we just need (B1). Similarly, one can check that the first seven paragraphs in B.13 below do not require the assumptions while (B1) suffices for the remainder of B.13 and for B.14.

B.13. In this and the next subsection we drop our assumption that $\chi\left(\mathfrak{b}^{+}\right)=0$ and consider arbitrary $\chi \in \mathfrak{g}^{*}$. (But we shall soon assume $\chi$ to be nilpotent.) Let $\mathcal{C}(\chi)$ denote the category of all finite dimensional $U_{\chi}(\mathfrak{g})$-modules; as before we just write $\mathcal{C}$ when it is clear which $\chi$ we consider.

Let $g \in G$. We write $\operatorname{Ad}(g)$ for the adjoint action of $g$ on $\mathfrak{g}$ and for the induced action on $U(\mathfrak{g})$. If $M$ is a $\mathfrak{g}$-module, then we write ${ }^{g} M$ for $M$ "twisted by $g$ ", i.e., we take ${ }^{g} M=M$ as a vector space and let any $x \in \mathfrak{g}$ (or in $\left.U(\mathfrak{g})\right)$ act on ${ }^{g} M$ as $\operatorname{Ad}\left(g^{-1}\right)(x)$ does on $M$. (See also the more general discussion at the beginning of [J2], 1.13.)

Clearly $M \mapsto{ }^{g} M$ is an exact functor that takes simple modules to simple modules. If $M$ is a $G$-module considered as a $\mathfrak{g}$-module via the derived action, then we have an isomorphism ${ }^{g} M \stackrel{\sim}{\longrightarrow} M$ given by $m \mapsto g m$. If $M$ is a $U_{\chi}(\mathfrak{g})$-module, then ${ }^{g} M$ is a $U_{g \chi}(\mathfrak{g})$-module where $g \chi$ is the image of $\chi$ under the coadjoint action of $g$ given by $(g \chi)(x)=\chi\left(\operatorname{Ad}\left(g^{-1}\right) x\right)$. The functor $M \mapsto{ }^{g} M$ is an equivalence of categories from $\mathcal{C}(\chi)$ to $\mathcal{C}(g \chi)$. It takes simple modules to simple modules.

Assume now that $\chi$ is nilpotent. This means that $\chi$ vanishes on a Borel subalgebra of $\mathfrak{g}$, or, equivalently, that there exists $g \in G$ with $(g \chi)\left(\mathfrak{b}^{+}\right)=0$; see [KW].

If $M$ is a $U_{\chi}(\mathfrak{g})$-module, then each $u \in U(\mathfrak{g})^{G}$ acts on each ${ }^{g} M$ as it does on $M$. Suppose for the moment that $(g \chi)\left(\mathfrak{b}^{+}\right)=0$; if $M$ is simple, then $U(\mathfrak{g})^{G}$ acts on ${ }^{g} M$ (and hence also on $M$ ) as on some $Z_{g \chi}(\lambda)$ with $\lambda \in X$. Set $\mathcal{C}(\chi)_{\lambda}$ equal to the full subcategory of $\mathcal{C}(\chi)$ consisting of those $N$ in $\mathcal{C}(\chi)$ such that $U(\mathfrak{g})^{G}$ acts on each composition factor of $N$ as on $Z_{g \chi}(\lambda)$. Then $\mathcal{C}(\chi)$ is the direct sum of all $\mathcal{C}(\chi)_{\lambda}$ with $\lambda$ in a suitable set of representatives.

Note: If we have already $\chi\left(\mathfrak{b}^{+}\right)=0$, then the definition of $\mathcal{C}(\chi)_{\lambda}$ given above coincides with the one that we get by applying B.1 directly, because each $u \in U(\mathfrak{g})^{G}$ acts on $Z_{\chi}(\lambda)$ and on $Z_{g \chi}(\lambda)$ by the same scalar (for each $\lambda \in X$ ); cf. [J2], 1.7. This observation implies for arbitrary $\chi$ that the definition of $\mathcal{C}(\chi)_{\lambda}$ is independent of the choice of $g$ with $(g \chi)\left(\mathfrak{b}^{+}\right)=0$.

We have (for each nilpotent $\chi$ ) projection functors $\operatorname{pr}_{\lambda}: \mathcal{C}(\chi) \rightarrow \mathcal{C}(\chi)_{\lambda}$ and translation functors $T_{\lambda}^{\mu}: \mathcal{C}(\chi)_{\lambda} \rightarrow \mathcal{C}(\chi)_{\mu}$ defined as in B.1 and B.2.

Let $g \in G$. If $M$ is a $U_{\chi}(\mathfrak{g})$-module in $\mathcal{C}(\chi)_{\lambda}$, then ${ }^{g} M$ belongs to $\mathcal{C}(g \chi)_{\lambda}$. The functor $M \mapsto{ }^{g} M$ restricts to an equivalence of categories from $\mathcal{C}(\chi)_{\lambda}$ to $\mathcal{C}(g \chi)_{\lambda}$. We get for arbitrary $M$ in $\mathcal{C}(\chi)$ that

$$
\operatorname{pr}_{\lambda}\left({ }^{g} M\right)={ }^{g}\left(\operatorname{pr}_{\lambda} M\right) .
$$


This implies for $M$ in $\mathcal{C}(\chi)_{\lambda}$ that

$$
{ }^{g}\left(T_{\lambda}^{\mu} M\right) \simeq T_{\lambda}^{\mu}\left({ }^{g} M\right) .
$$

Indeed, we use the same $G$-module $E$ when we define $T_{\lambda}^{\mu}$ on $\mathcal{C}(\chi)_{\lambda}$ and on $\mathcal{C}(g \chi)_{\lambda}$ and therefore get

$$
\begin{aligned}
{ }^{g}\left(T_{\lambda}^{\mu} M\right) & ={ }^{g}\left(\operatorname{pr}_{\mu}(E \otimes M)\right)=\operatorname{pr}_{\mu}{ }^{g}(E \otimes M)=\operatorname{pr}_{\mu}\left({ }^{g} E \otimes{ }^{g} M\right) \\
& \simeq \operatorname{pr}_{\mu}\left(E \otimes{ }^{g} M\right)=T_{\lambda}^{\mu}\left({ }^{g} M\right) .
\end{aligned}
$$

Lemma. Suppose that $\chi \in \mathfrak{g}^{*}$ and $g \in G$ with $\chi\left(\mathfrak{b}^{+}\right)=(g \chi)\left(\mathfrak{b}^{+}\right)=0$. Let $\lambda \in X$ and let $L$ be a simple $U_{\chi}(\mathfrak{g})$-module. Then

$$
\left[Z_{g \chi}(\lambda):{ }^{g} L\right]=\left[Z_{\chi}(\lambda): L\right]
$$

Proof. If $Q_{L}$ is the projective cover of $L$ in the category of all $U_{\chi}(\mathfrak{g})$-modules, then ${ }^{g}\left(Q_{L}\right)$ is the projective cover of $L$ in the category of all $U_{g \chi}(\mathfrak{g})$-modules. Applying B.12(2) to $g \chi$ instead of $\chi$, we get

$$
\operatorname{dim}{ }^{g}\left(Q_{L}\right)=p^{N}|W \cdot(\lambda+p X)|\left[Z_{g \chi}(\lambda):{ }^{g} L\right] .
$$

Since $Q_{L}$ and ${ }^{g}\left(Q_{L}\right)$ have the same dimension, a comparison of (4) with B.12(1) yields (3).

B.14. Let again $g \in G$. Given a Lie subalgebra $\mathfrak{q}$ of $\mathfrak{g}$ and a $\mathfrak{q}$-module $M$, then we get an $\operatorname{Ad}(g) \mathfrak{q}$-module ${ }^{g} M$ by an obvious generalisation of the definition in B.13. If $\mathfrak{q}$ is a restricted Lie subalgebra of $\mathfrak{g}$ and if $M$ is a $U_{\chi}(\mathfrak{q})$-module, then ${ }^{g} M$ is a $U_{g \chi}(\operatorname{Ad}(g) \mathfrak{q})$-module. It is then easy to check that we get for all $\chi \in \mathfrak{g}^{*}$ an isomorphism for the induced modules

$$
{ }^{g}\left(U_{\chi}(\mathfrak{g}) \otimes_{U_{\chi}(\mathfrak{q})} M\right) \stackrel{\sim}{\longrightarrow} U_{g \chi}(\mathfrak{g}) \otimes_{U_{g \chi}(\operatorname{Ad}(g) \mathfrak{q})}{ }^{g} M
$$

induced by $u \otimes m \mapsto \operatorname{Ad}(g)(u) \otimes m$.

Let $B^{+}=P_{\emptyset}$ (cf. B.6) be the Borel subgroup of $G$ with Lie algebra $\mathfrak{b}^{+}$. If $\chi \in \mathfrak{g}^{*}$ with $\chi\left(\mathfrak{b}^{+}\right)=0$, then we get applying (1) with $\mathfrak{q}=\mathfrak{b}^{+}$

$$
{ }^{g} Z_{\chi}(\lambda) \simeq Z_{g \chi}(\lambda) \quad \text { for all } \lambda \in X \text { and } g \in B^{+}
$$

since $\operatorname{Ad}(g)\left(\mathfrak{b}^{+}\right)=\mathfrak{b}^{+}$and since $\operatorname{Ad}(g)$ acts trivially on $\mathfrak{b}^{+} / \mathfrak{n}^{+}$.

Let $\alpha$ be a simple root and let $P_{\alpha} \supset B^{+}$be the standard parabolic subgroup with Lie algebra $\mathfrak{p}_{\alpha}=\mathfrak{b}^{+}+\mathfrak{g}_{-\alpha}$. Suppose that $\chi\left(\mathfrak{p}_{\alpha}\right)=0$. Then we claim that

$$
{ }^{g} Z_{\chi}(\lambda, \alpha) \simeq Z_{g \chi}(\lambda, \alpha) \quad \text { for all } \lambda \in X \text { and } g \in P_{\alpha} .
$$

We want to apply (1) using A.4(4). We can replace $\lambda$ by a weight in $\lambda+p X$ and assume that $0 \leq\left\langle\lambda, \alpha^{\vee}\right\rangle<p$. Then we get $L_{\chi, \alpha}(\lambda)$ by taking the derived action of $\mathfrak{p}_{\alpha}$ on the simple $P_{\alpha}$-module with highest weight $\lambda$. This then implies ${ }^{g} L_{\chi, \alpha}(\lambda) \simeq L_{\chi, \alpha}(\lambda)$, hence via (1) the claim.

$$
\text { C. }
$$

Keep all assumptions and notations from Section B, in particular (B1) and (B2). However, one may check that (B1) and (B2) are used only for C.1-C.4 and C.9C.10.

We introduced in A.1 an element $\rho \in X \otimes_{\mathbf{z}} \mathbf{Q}$. If (B1) holds, then we can choose $\rho \in X$. We assume in the future that we have made such a choice whenever (B1) holds. (If $G$ is not semi-simple, then $\rho$ is not necessarily half the sum of the positive roots.) 
C.1. Set

$$
C_{0}=\left\{\lambda \in X \mid 0 \leq\left\langle\lambda+\rho, \beta^{\vee}\right\rangle \leq p \text { for all } \beta \in R^{+}\right\}
$$

and

$$
C_{0}^{\prime}=\left\{\lambda \in X \mid 0 \leq\left\langle\lambda+\rho, \beta^{\vee}\right\rangle<p \text { for all } \beta \in R^{+}\right\} .
$$

So $C_{0}$ is the usual "first dominant alcove" $C_{0}$ as in [J3], 11.19(1) or [J4], 4.1(1).

We begin now (for certain $\chi$ ) an investigation of the subcategories $\mathcal{C}_{\lambda}$ with $\lambda \in$ $C_{0}^{\prime}$. If $R$ has no components of exceptional type, then one can show (see H.1 below) that there exists for each $\mu \in X$ a weight $\lambda \in C_{0}^{\prime}$ with $\mu \in W \bullet \lambda+p X$, hence with $\mathcal{C}_{\mu}=\mathcal{C}_{\lambda}$. So in those cases we do not lose anything by the restriction to weights in $C_{0}^{\prime}$.

Lemma. Let $\lambda \in C_{0}^{\prime}$. If $w \in W$ and $\nu \in X$ with $w_{\bullet} \lambda+p \nu=\lambda$, then $\nu=0$.

Proof. If $w_{\bullet} \lambda+p \nu=\lambda$, then $p \nu \in \mathbf{Z} R$ since $w_{\bullet} \lambda \in \lambda+\mathbf{Z} R$. So our assumption (B2) implies that $\nu \in \mathbf{Z} R$. This means that the map $x \mapsto w \cdot x+p \nu$ belongs to the stabiliser of $\lambda$ in the affine Weyl group $W_{p}$. Since $W_{p}$ is a reflection group, that stabiliser is generated by all $s_{\alpha, r p}$ with $\alpha \in R^{+}$and $\left\langle\lambda+\rho, \alpha^{\vee}\right\rangle=r p$. The definition of $C_{0}^{\prime}$ implies that then necessarily $r p=0$. Therefore the stabiliser of $\lambda$ in $W_{p}$ is contained in $W$; this yields $\nu=0$.

C.2. Proposition. Let $\lambda \in C_{0}^{\prime}$. There exists a simple module $L$ in $\mathcal{C}_{\lambda}$ with projective cover isomorphic to $T_{-\rho}^{\lambda} Z_{\chi}(-\rho)$; it satisfies $\left[Z_{\chi}(\lambda): L\right]=1$.

Proof. Let us abbreviate $Q=T_{-\rho}^{\lambda} Z_{\chi}(-\rho)$. Proposition B.3 implies that $Q$ has a filtration with factors $Z_{\chi}(\mu)$ with $\mu \in W \cdot \lambda$, one factor for each such $\mu$. We get in particular that

$$
\operatorname{dim} Q=|W \cdot \lambda| p^{N}
$$

with $N=\left|R^{+}\right|$.

One knows that $Z_{\chi}(-\rho)$ is projective; cf. [FP2], Thm. 4.1. Any translation functor $T_{\mu}^{\nu}$ takes projective modules to projective modules since the adjoint functor $T_{\nu}^{\mu}$ is exact. Therefore $Q$ is projective.

Let $L$ be a simple quotient of $Q$. Then the projective cover $Q_{L}$ of $L$ has to be a direct summand of $Q$. We have by B.12(2)

$$
\operatorname{dim} Q_{L}=p^{N}|W \cdot(\lambda+p X)|\left[Z_{\chi}(\lambda): L\right] .
$$

Now Lemma C.1 implies that the stabiliser of $\lambda+p X$ in $W$ is equal to the stabiliser of $\lambda$ in $W$. Using (1) and the fact that $Q_{L}$ is a summand of $Q$, we get that

$$
\operatorname{dim} Q=p^{N}|W \cdot \lambda| \geq \operatorname{dim} Q_{L}=p^{N}|W \cdot \lambda|\left[Z_{\chi}(\lambda): L\right] \geq p^{N}|W \cdot \lambda| .
$$

So we have to have equality everywhere, hence $Q_{L}=Q$ and $\left[Z_{\chi}(\lambda): L\right]=1$. So the claim follows.

C.3. Proposition C.2 yields a representation theoretic proof of the following special case of a recent theorem of Brown and Gordon in [BG], 3.18:

Corollary. If $\lambda \in C_{0}^{\prime}$, then the subcategory $\mathcal{C}_{\lambda}$ is a block of the category of all finite dimensional $U_{\chi}(\mathfrak{g})$-modules. 
Proof. If $\mathcal{C}_{\lambda}$ is not a block, then it is a direct sum of two non-trivial subcategories that are closed under taking subquotients. The indecomposable module $Q$ considered in C.2 would have to belong to one of them. Then so would all subquotients $Z_{\chi}(w \bullet \lambda)$ with $w \in W$ of $Q$, hence all simple modules in $\mathcal{C}_{\lambda}$. Then the other subcategory will contain no simple modules at all, hence be trivial.

Remark. The theorem in [BG] says that all $\mathcal{C}_{\mu}$ with $\mu \in X$ are blocks. That proves the conjecture by Humphreys in $[\mathrm{H}]$, Section 18. (For arbitrary type such a result had previously been known only for $\chi$ in standard Levi form; cf. $[H]$.) The corollary here together with H.1 yields that conjecture in case $R$ has no components of exceptional type (and $p \neq 2$ if it has components not of type $A$ ).

C.4. Note that a simple module over a finite dimensional algebra is determined up to isomorphism by its projective cover. In particular, the module $L$ in Proposition C.2 is unique up to isomorphism. We shall refer to it as the simple module in $\mathcal{C}_{\lambda}$ with projective cover isomorphic to $T_{-\rho}^{\lambda} Z_{\chi}(-\rho)$.

Let $w_{0} \in W$ denote the unique element with $w_{0}\left(R^{+}\right)=-R^{+}$.

Proposition. Let $\lambda \in C_{0}^{\prime}$, let $L$ denote the simple module in $\mathcal{C}_{\lambda}$ with projective cover isomorphic to $T_{-\rho}^{\lambda} Z_{\chi}(-\rho)$.

a) Up to isomorphism $L$ is the only simple module in $\mathcal{C}_{\lambda}$ with $T_{\lambda}^{-\rho} L \neq 0$.

b) The socle of $Z_{\chi}(\lambda)$ and the head of $Z_{\chi}\left(w_{0} \bullet \lambda\right)$ are isomorphic to $L$. We have

$$
\operatorname{dim} \operatorname{Hom}_{\mathfrak{g}}\left(Z_{\chi}\left(w_{0} \bullet \lambda\right), Z_{\chi}(\lambda)\right)=1 \text {. }
$$

Each non-zero homomorphism from $Z_{\chi}\left(w_{0} \cdot \lambda\right)$ to $Z_{\chi}(\lambda)$ has image equal to the socle of $Z_{\chi}(\lambda)$.

Proof. a) It is known that $Z_{\chi}(-\rho)$ is simple; cf. [FP2], Thm. 4.2. (The assumption in that theorem that $p$ is good for $R$ is not needed for this particular result.) Therefore $Z_{\chi}(-\rho)$ is the only simple module in $\mathcal{C}_{-\rho}$ (up to isomorphism). If $M$ is a module in $\mathcal{C}_{\lambda}$ with $T_{\lambda}^{-\rho} M \neq 0$, then we get

$$
0 \neq \operatorname{Hom}_{\mathfrak{g}}\left(Z_{\chi}(-\rho), T_{\lambda}^{-\rho} M\right) \simeq \operatorname{Hom}_{\mathfrak{g}}\left(T_{-\rho}^{\lambda} Z_{\chi}(-\rho), M\right) .
$$

If we assume additionally that $M$ is simple, then we get that $M$ is a homomorphic image of the projective cover of $L$, hence isomorphic to $L$.

b) Apply the discussion preceding Proposition B.3 to $Q=T_{-\rho}^{\lambda} Z_{\chi}(-\rho)$. It follows that $Z_{\chi}(\lambda)$ is a submodule of $Q$ and that $Z_{\chi}\left(w_{0} \bullet \lambda\right)$ is a factor module. Since $Q$ (being the projective cover of $L$ ) has simple head isomorphic to $L$, so has $Z_{\chi}\left(w_{0} \bullet \lambda\right)$. Since $U_{\chi}(\mathfrak{g})$ is a symmetric algebra (see [FP1], Prop. 1.2), the simple module $L$ is also isomorphic to the socle of $Q$, hence to that of $Z_{\chi}(\lambda)$.

It is now clear that we get a non-zero homomorphism from $Z_{\chi}\left(w_{0} \bullet \lambda\right)$ to $Z_{\chi}(\lambda)$ by projecting first onto $L$ and then embedding $L$ into $Z_{\chi}(\lambda)$. Conversely, let $\varphi$ be a non-zero homomorphism from $Z_{\chi}\left(w_{0} \bullet \lambda\right)$ to $Z_{\chi}(\lambda)$. The image of $\varphi$ has simple head and simple socle, both isomorphic to $L$. Since $\left[Z_{\chi}(\lambda): L\right]=1$, the image of $\varphi$ has to be isomorphic to $L$. Therefore the image is equal to the socle of $Z_{\chi}(\lambda)$, and the kernel of $\varphi$ has to be equal to the radical of $Z_{\chi}\left(w_{0} \bullet \lambda\right)$. Now (1) follows from Schur's Lemma.

Remark. The entries in the row (or column) of the Cartan matrix of $\mathcal{C}_{\lambda}$ corresponding to $L$ are equal to $|W \cdot(\lambda+p X)|\left[Z_{\chi}(\lambda): L^{\prime}\right]$ with $L^{\prime}$ running over the simple modules in $\mathcal{C}_{\lambda}$. In particular, the diagonal entry is equal to $|W \cdot(\lambda+p X)|$ and the 
other entries are non-zero multiples of that number. This implies that Lusztig's original conjecture in [L2], 14.5 for the Cartan matrix could not work in type $D_{4}$ (for example).

C.5. Fix $\lambda \in C_{0}^{\prime}$. If $w \in W$ and if $\alpha$ is a simple root with $w^{-1} \alpha>0$, then $0 \leq\left\langle w(\lambda+\rho), \alpha^{\vee}\right\rangle<p$ and we have a homomorphism

$$
\varphi_{w, s_{\alpha} w}^{\lambda}: Z_{\chi}\left(s_{\alpha} w \cdot \lambda\right) \longrightarrow Z_{\chi}(w \cdot \lambda)
$$

given by

$$
\varphi_{w, s_{\alpha} w}^{\lambda}\left(v_{s_{\alpha} w \bullet \lambda}\right)=x_{-\alpha}^{\left\langle w(\lambda+\rho), \alpha^{\vee}\right\rangle} v_{w \bullet \lambda} .
$$

If $\left\langle w(\lambda+\rho), \alpha^{\vee}\right\rangle=0$, then $\varphi_{w, s_{\alpha} w}^{\lambda}$ is the identity map on $Z_{\chi}(\lambda)$. If $\left\langle w(\lambda+\rho), \alpha^{\vee}\right\rangle>$ 0 , then $\varphi_{w, s_{\alpha} w}^{\lambda}$ is up to a non-zero scalar multiple of the map $\varphi_{\alpha}^{w \bullet \lambda}$ from A.4(2). We get therefore from A.4:

Lemma. If $\chi\left(x_{-\alpha}\right) \neq 0$, then $\varphi_{w, s_{\alpha} w}^{\lambda}$ is an isomorphism. If $\chi\left(x_{-\alpha}\right)=0$, then

$$
\operatorname{im} \varphi_{w, s_{\alpha} w}^{\lambda} \simeq Z_{\chi}\left(s_{\alpha} w \cdot \lambda, \alpha\right)
$$

and

$$
\operatorname{dim} \operatorname{im}\left(\varphi_{w, s_{\alpha} w}^{\lambda}\right)=\left(p-\left\langle w(\lambda+\rho), \alpha^{\vee}\right\rangle\right) p^{N-1}
$$

where $N=\left|R^{+}\right|$.

C.6. We now want to define for each $w \in W$ a homomorphism

$$
\varphi_{w}^{\lambda}: Z_{\chi}(w \cdot \lambda) \longrightarrow Z_{\chi}(\lambda)
$$

as follows: If $w=s_{1} s_{2} \ldots s_{r}$ is a reduced decomposition (with $s_{i}=s_{\alpha_{i}}$ for some simple root $\alpha_{i}$ ), then we want to have

$$
\varphi_{w}^{\lambda}=\varphi_{1, s_{r}}^{\lambda} \circ \varphi_{s_{r}, s_{r-1} s_{r}}^{\lambda} \circ \cdots \circ \varphi_{s_{2} \ldots s_{r-1} s_{r}, s_{1} s_{2} \ldots s_{r-1} s_{r}}^{\lambda}
$$

where the single factors are defined in C.5. One has to check the independence of the right hand side in (2) of the chosen reduced decomposition. It is (as usual) enough to check the "braid relations" for each pair $\alpha, \beta,(\alpha \neq \beta)$ of simple roots. For example, if $s_{\alpha} s_{\beta}$ has order 3 , then we have to check for each $x \in W$ with $x^{-1} \alpha, x^{-1} \beta>0$ that

$$
\varphi_{x, s_{\alpha} x}^{\lambda} \circ \varphi_{s_{\alpha} x, s_{\beta} s_{\alpha} x}^{\lambda} \circ \varphi_{s_{\beta} s_{\alpha} x, s_{\alpha} s_{\beta} s_{\alpha} x}^{\lambda}=\varphi_{x, s_{\beta} x}^{\lambda} \circ \varphi_{s_{\beta} x, s_{\alpha} s_{\beta} x}^{\lambda} \circ \varphi_{s_{\alpha} s_{\beta} x, s_{\beta} s_{\alpha} s_{\beta} x}^{\lambda} .
$$

This follows from the Verma relations (see [D], 7.8.8); similarly in the cases where $s_{\alpha} s_{\beta}$ has order 2,4 , or 6 .

Now that the $\varphi_{w}^{\lambda}$ are well-defined, we get for all $w \in W$ and all simple roots $\alpha$ with $w^{-1} \alpha>0$ that

$$
\varphi_{s_{\alpha} w}^{\lambda}=\varphi_{w}^{\lambda} \circ \varphi_{w, s_{\alpha} w}^{\lambda}
$$

since we get a reduced decomposition of $s_{\alpha} w$ when we multiply a reduced decomposition of $W$ on the left by $s_{\alpha}$. 
C.7. $\quad$ Set for all $w \in W$

$$
\operatorname{sbm}(w, \lambda)=\operatorname{im}\left(\varphi_{w}^{\lambda}\right) \subset Z_{\chi}(\lambda)
$$

the submodule corresponding to $w$.

Lemma. Let $\alpha$ be a simple root and $w$ an element in $W$ with $w^{-1} \alpha>0$.

a) We have $\operatorname{sbm}\left(s_{\alpha} w, \lambda\right) \subset \operatorname{sbm}(w, \lambda)$.

b) If $\chi\left(x_{-\alpha}\right) \neq 0$, then $\operatorname{sbm}\left(s_{\alpha} w, \lambda\right)=\operatorname{sbm}(w, \lambda)$.

c) If $\chi\left(x_{-\alpha}\right)=0$, then $\operatorname{sbm}\left(s_{\alpha} w, \lambda\right)$ is a homomorphic image of $Z_{\chi}\left(s_{\alpha} w \cdot \lambda, \alpha\right)$, and $\operatorname{sbm}(w, \lambda) / \operatorname{sbm}\left(s_{\alpha} w, \lambda\right)$ is a homomorphic image of $Z_{\chi}(w \cdot \lambda, \alpha)$.

Proof. The identity $\varphi_{s_{\alpha} w}^{\lambda}=\varphi_{w}^{\lambda} \circ \varphi_{w, s_{\alpha} w}^{\lambda}$ from C.6(3) implies a). If $\chi\left(x_{-\alpha}\right) \neq 0$, then $\varphi_{w, s_{\alpha} w}^{\lambda}$ is an isomorphism; this yields b).

Suppose now that $\chi\left(x_{-\alpha}\right)=0$. Denote the image of $\varphi_{w, s_{\alpha} w}^{\lambda}$ by $M$. Then $M$ is isomorphic to $Z_{\chi}\left(s_{\alpha} w \bullet \lambda, \alpha\right)$, and $Z_{\chi}(w \bullet \lambda) / M$ is isomorphic to $Z_{\chi}(w \bullet \lambda, \alpha)$. Now the claim in c) follows from $\operatorname{sbm}\left(s_{\alpha} w, \lambda\right)=\varphi_{w}^{\lambda}(M)$ and $\operatorname{sbm}(w, \lambda)=\varphi_{w}^{\lambda}\left(Z_{\chi}(w \cdot \lambda)\right)$.

Remark. It is sometimes more convenient to restate the last part of the lemma as follows: Let $w \in W$ and $\gamma \in R^{+}$such that $w \gamma$ is a simple root with $\chi\left(x_{-w \gamma}\right)=0$. Then $\operatorname{sbm}\left(w s_{\gamma}, \lambda\right)$ is a homomorphic image of $Z_{\chi}\left(w s_{\gamma} \cdot \lambda, w \gamma\right)$, and $\operatorname{sbm}(w, \lambda) / \operatorname{sbm}$ $\left(w s_{\gamma}, \lambda\right)$ is a homomorphic image of $Z_{\chi}(w \bullet \lambda, \alpha)$. [Note that $s_{w \gamma} w=w s_{\gamma}$.]

C.8. We now want to generalise Lemma C.7.a to positive roots that are not necessarily simple:

Proposition. Let $w \in W$ and $\alpha \in R^{+}$with $w^{-1} \alpha>0$. Then $\operatorname{sbm}\left(s_{\alpha} w, \lambda\right) \subset$ $\operatorname{sbm}(w, \lambda)$.

Proof. This will follow in the same way as in C.7 if we can find a homomorphism

$$
\varphi_{w, s_{\alpha} w}^{\lambda}: Z_{\chi}\left(s_{\alpha} w \cdot \lambda\right) \rightarrow Z_{\chi}\left(w_{\bullet} \lambda\right) \quad \text { with } \quad \varphi_{s_{\alpha} w}^{\lambda}=\varphi_{w}^{\lambda} \circ \varphi_{w, s_{\alpha} w}^{\lambda} .
$$

Suppose first that $G$ is semi-simple and simply connected; drop the assumption that (B2) should hold. In order to construct the map in (1) we make a detour to characteristic 0 . Let $\mathfrak{g}_{\mathbf{C}}$ be a complex semisimple Lie algebra of the same type as $\mathfrak{g}$. Fix a triangular decomposition $\mathfrak{g}_{\mathbf{C}}=\mathfrak{n}_{\mathbf{C}}^{-} \oplus \mathfrak{h}_{\mathbf{C}} \oplus \mathfrak{n}_{\mathbf{C}}^{+}$and a Chevalley basis of $\mathfrak{g}_{\mathbf{C}}$. Denote by $\mathfrak{g}_{\mathbf{Z}}$ the span over $\mathbf{Z}$ of our Chevalley basis. This is a Lie algebra over $\mathbf{Z}$ with a triangular decomposition $\mathfrak{g}_{\mathbf{Z}}=\mathfrak{n}_{\mathbf{Z}}^{-} \oplus \mathfrak{h}_{\mathbf{Z}} \oplus \mathfrak{n}_{\mathbf{Z}}^{+}$induced by that of $\mathfrak{g}_{\mathbf{C}}$. We can and shall assume that $\mathfrak{g}=\mathfrak{g}_{\mathbf{Z}} \otimes \mathbf{z} K$, similarly for $\mathfrak{n}^{ \pm}$and $\mathfrak{h}$. We have then also $U(\mathfrak{g})=U\left(\mathfrak{g}_{\mathbf{z}}\right) \otimes_{\mathbf{z}} K$ and similarly for $\mathfrak{n}^{ \pm}$and $\mathfrak{h}$. We denote (by abuse of notation) the vectors in our Chevalley basis of $\mathfrak{g}_{\mathbf{C}}$ by $x_{\gamma}$ and $h_{\beta}(\beta, \gamma \in R, \beta$ simple). We assume that we have chosen the $x_{\gamma} \otimes 1$ as our root vectors in $\mathfrak{g}$ (which we usually denote by $x_{\gamma}$ ).

The group $X$ can be identified with the lattice of integral weights of $\mathfrak{h}_{\mathbf{C}}$. We have for each $\nu \in X$ a Verma module $M(\nu)_{\mathbf{C}}$ for $\mathfrak{g}_{\mathbf{C}}$ with highest weight $\nu$; we denote its standard generator by $z_{\nu}$. We define for each $w \in W$ a homomorphism $f_{w}^{\lambda}: M(w \cdot \lambda)_{\mathbf{C}} \rightarrow M(\lambda)_{\mathbf{C}}$ in the same way as we defined in C.6(2) the $\varphi_{w}^{\lambda}$. There is a unique element

$$
u_{w}^{\lambda} \in U\left(\mathfrak{n}_{\mathbf{C}}^{-}\right) \quad \text { with } \quad f_{w}^{\lambda}\left(z_{w \bullet \lambda}\right)=u_{w}^{\lambda} z_{\lambda} .
$$

The construction shows that $u_{w}^{\lambda}$ is a product of powers of the $x_{-\beta}$ with $\beta$ simple, hence contained in $U\left(\mathfrak{n}_{\mathbf{Z}}^{-}\right)$, and that

$$
\varphi_{w}^{\lambda}\left(v_{w \bullet \lambda}\right)=\left(u_{w}^{\lambda} \otimes 1\right) v_{\lambda} .
$$


Let $\beta_{1}, \beta_{2}, \ldots, \beta_{n}$ denote the simple roots. If we write $u_{w}^{\lambda}$ as a linear combination of the usual PBW basis of $U\left(\mathfrak{n}_{\mathbf{C}}^{-}\right)$, then

$$
u_{w}^{\lambda}=\prod_{i=1}^{n} x_{-\beta_{i}}^{r_{i}}+\text { lower order terms } \quad \text { if } \lambda-w_{\bullet} \lambda=\sum_{i=1}^{n} r_{i} \beta_{i}
$$

where "lower order terms" refers to the canonical filtration of an enveloping algebra as in $[\mathrm{D}], 2.3 .1$.

Now consider $w$ and $\alpha$ as in the proposition. The theory of Verma modules shows that there exists a unique homomorphism

$$
f_{w, s_{\alpha} w}^{\lambda}: M\left(s_{\alpha} w \cdot \lambda\right)_{\mathbf{C}} \rightarrow M(w \cdot \lambda)_{\mathbf{C}} \quad \text { with } \quad f_{w}^{\lambda} \circ f_{w, s_{\alpha} w}^{\lambda}=f_{s_{\alpha} w}^{\lambda},
$$

see [D], 7.6.6 and 7.6.23. There is a unique element

$$
u_{w, s_{\alpha} w}^{\lambda} \in U\left(\mathfrak{n}_{\mathbf{C}}^{-}\right) \quad \text { with } \quad f_{w, s_{\alpha} w}^{\lambda}\left(z_{s_{\alpha} w \bullet \lambda}\right)=u_{w, s_{\alpha} w}^{\lambda} z_{w \bullet \lambda} .
$$

A comparison with (2) shows that

$$
u_{s_{\alpha} w}^{\lambda}=u_{w, s_{\alpha} w}^{\lambda} u_{w}^{\lambda} .
$$

A look at the terms of highest order shows that

$$
u_{w, s_{\alpha} w}^{\lambda}=\prod_{i=1}^{n} x_{-\beta_{i}}^{r_{i}}+\text { lower order terms } \quad \text { if } w_{\bullet} \lambda-s_{\alpha} w_{\bullet} \lambda=\sum_{i=1}^{n} r_{i} \beta_{i} .
$$

If we write $d=\left\langle w(\lambda+\rho), \alpha^{\vee}\right\rangle$, then we have above $d \alpha=\sum_{i=1}^{n} r_{i} \beta_{i}$. This shows that $u_{w, s_{\alpha} w}^{\lambda}$ is equal to the element denoted by $S_{\alpha, d}\left(w_{\bullet} \lambda\right)$ in [F], Section 3. (Note that there is a misprint in the last displayed equation on p. 66 of $[\mathrm{F}]$ : One should replace $r$ by $d r$.)

The results in $[\mathrm{F}]$ say that

$$
u_{w, s_{\alpha} w}^{\lambda} \in U\left(\mathfrak{n}_{\mathbf{Z}}^{-}\right) .
$$

(See the remarks on the top of p. 67 in $[\mathrm{F}]$.) We now want to use this element to define $\varphi_{w, s_{\alpha} w}^{\lambda}$ by

$$
\varphi_{w, s_{\alpha} w}^{\lambda}\left(v_{s_{\alpha} w \bullet \lambda}\right)=\left(u_{w, s_{\alpha} w}^{\lambda} \otimes 1\right) v_{w \bullet \lambda} .
$$

If this is possible, then (7) and (3) yield the equality $\varphi_{s_{\alpha} w}^{\lambda}=\varphi_{w}^{\lambda} \circ \varphi_{w, s_{\alpha} w}^{\lambda}$, hence the proposition.

The right hand side in (10) has weight $s_{\alpha} w_{\bullet} \lambda$; it therefore suffices to show that this term is annihilated by all $x_{\gamma} \otimes 1$ with $\gamma>0$. We have to start with in $U\left(\mathfrak{g}_{\mathbf{z}}\right)$

$$
x_{\gamma} u_{w, s_{\alpha} w}^{\lambda}=\sum_{\pi} F_{\pi} c_{\pi}+\text { terms in } U\left(\mathfrak{g}_{\mathbf{Z}}\right) \mathfrak{n}_{\mathbf{z}}^{+}
$$

where the $F_{\pi}$ are (as in $[\mathrm{F}]$ ) the elements in a PBW basis of $U\left(\mathfrak{n}_{\mathbf{Z}}^{-}\right)$and where all $c_{\pi} \in U\left(\mathfrak{h}_{\mathbf{z}}\right)$. We have then

$$
0=x_{\gamma} u_{w, s_{\alpha} w}^{\lambda} z_{w \bullet \lambda}=\sum_{\pi}(w \bullet \lambda)\left(c_{\pi}\right) F_{\pi} z_{w \bullet \lambda} .
$$

Since the $F_{\pi} z_{w \bullet \lambda}$ are linearly independent, we get $\left(w_{\bullet} \lambda\right)\left(c_{\pi}\right)=0$ for all $\pi$. Since

$$
\left(x_{\gamma} \otimes 1\right)\left(u_{w, s_{\alpha} w}^{\lambda} \otimes 1\right) v_{w \bullet \lambda}=\sum_{\pi}\left(w_{\bullet} \lambda\right)\left(c_{\pi}\right)\left(F_{\pi} \otimes 1\right) v_{w \bullet \lambda}=0
$$

the claim follows (for $G$ semi-simple and simply connected). 
The extension to the case where $G$ is a direct product of a semi-simple and simply connected group with a torus is immediate. In general, $G$ is a quotient of such a group, say $\widetilde{G}$, by a central subgroup. The corresponding homomorphism $\pi$ from $\widetilde{\mathfrak{g}}=\operatorname{Lie}(\widetilde{G})$ to $\mathfrak{g}$ satisfies $\mathfrak{g}=\mathfrak{h}+\pi(\widetilde{\mathfrak{g}})$. If we consider a baby Verma module for $\mathfrak{g}$ as a $\widetilde{\mathfrak{g}}$-module via $\pi$, we get a baby Verma module for $\widetilde{\mathfrak{g}}$. We can use the construction above to get a map $\varphi_{w, s_{\alpha} w}^{\lambda}$ as in (1) that is a homomorphism of $\widetilde{\mathfrak{g}}$-modules. It then suffices to show that this map also commutes with $\mathfrak{h}$. That, however, follows from the fact that the element $u_{w, s_{\alpha} w}^{\lambda}$ has weight $-d \alpha$ also for $\mathfrak{h}$.

Remark. Let $\leq$ denote the usual Chevalley-Bruhat order on $W$ (with smallest element 1). The proposition implies for all $w_{1}, w_{2} \in W$ :

$$
w_{1} \geq w_{2} \Longrightarrow \operatorname{sbm}\left(w_{1}, \lambda\right) \subset \operatorname{sbm}\left(w_{2}, \lambda\right) .
$$

C.9. Lemma. Let $\mu$ be a weight in the closure of the facet of $\lambda$ with $\mu \in C_{0}^{\prime}$. For all $w \in W$ and $\alpha \in R$ simple and $w^{-1} \alpha>0$ we can identify $T_{\lambda}^{\mu}\left(\varphi_{w, s_{\alpha} w}^{\lambda}\right)$ with a non-zero multiple of $\varphi_{w, s_{\alpha} w}^{\mu}$. We can identify $T_{\lambda}^{\mu}\left(\varphi_{w}^{\lambda}\right)$ with a non-zero multiple of $\varphi_{w}^{\mu}$ and have

$$
T_{\lambda}^{\mu} \operatorname{sbm}(w, \lambda) \simeq \operatorname{sbm}(w, \mu)
$$

for all $w \in W$.

Proof. In order to prove the claim for the $\varphi_{w, s_{\alpha} w}^{\lambda}$ we distinguish two cases:

If $\left\langle w(\lambda+\rho), \alpha^{\vee}\right\rangle=0$, then the assumption on $\mu$ implies that also $\left\langle w(\mu+\rho), \alpha^{\vee}\right\rangle=$ 0 . Then both $\varphi_{w, s_{\alpha} w}^{\lambda}$ and $\varphi_{w, s_{\alpha} w}^{\mu}$ are the identity. So is $T_{\lambda}^{\mu}\left(\varphi_{w, s_{\alpha} w}^{\lambda}\right)$; the claim follows in this case.

If $\left\langle w(\lambda+\rho), \alpha^{\vee}\right\rangle>0$, then the claim follows easily from Proposition B.10 because $\varphi_{w, s_{\alpha} w}^{\lambda}$ is a non-zero multiple of the map $\varphi_{\alpha}^{w \bullet \lambda}$ considered there.

The rest of the lemma follows now from the definition of $\varphi_{w}^{\lambda}$, the functor property of $T_{\lambda}^{\mu}$ and its exactness.

C.10. We can apply Lemma C.9 to $\mu=-\rho$. Since all $\varphi_{w}^{-\rho}$ are the identity, hence non-zero, we get that

$$
\varphi_{w}^{\lambda} \neq 0 \quad \text { for all } w \in W .
$$

This holds in particular for $w=w_{0}$. Proposition C.4.b implies therefore that

$$
\operatorname{sbm}\left(w_{0}, \lambda\right)=\operatorname{soc} Z_{\chi}(\lambda) \text {. }
$$

C.11. Set

$$
I=\left\{\alpha \in R \mid \alpha \text { simple, } \chi\left(x_{-\alpha}\right) \neq 0\right\} .
$$

Let $W_{I}$ denote the subgroup of $W$ generated by all $s_{\alpha}$ with $\alpha \in I$.

Lemma. a) We have $\operatorname{sbm}\left(w_{1}, \lambda\right)=\operatorname{sbm}\left(w_{2}, \lambda\right)$ for all $w_{1}, w_{2} \in W$ with $W_{I} w_{1}=$ $W_{I} w_{2}$.

b) Let $w \in W_{I}$ and $\alpha$ be a simple root with $\alpha \notin I$. Then

$$
\operatorname{sbm}\left(s_{\alpha} w, \lambda\right) \simeq Z_{\chi}\left(s_{\alpha} w \cdot \lambda, \alpha\right)
$$

and

$$
\operatorname{dim} \operatorname{sbm}\left(s_{\alpha} w, \lambda\right)=\left(p-\left\langle w(\lambda+\rho), \alpha^{\vee}\right\rangle\right) p^{N-1}
$$

where $N=\left|R^{+}\right|$. 
Proof. The claim in a) follows easily from Lemma C.7.b. In b) we have $w^{-1} \alpha>0$ since $w \in W_{I}$ and $\alpha \notin I$. It follows that $\varphi_{s_{\alpha} w}^{\lambda}=\varphi_{w}^{\lambda} \circ \varphi_{w, s_{\alpha} w}^{\lambda}$. Furthermore $\varphi_{w}^{\lambda}$ is a composition of certain $\varphi_{w^{\prime}, s_{\beta} w^{\prime}}^{\lambda}$ with $\beta \in I$, hence an isomorphism. Therefore the image $\operatorname{sbm}\left(s_{\alpha} w, \lambda\right)$ of $\varphi_{s_{\alpha} w}^{\lambda}$ is isomorphic to the image of $\varphi_{w, s_{\alpha} w}^{\lambda}$. Now the claims in b) follow from Lemma C.5.

C.12. Here and in the next two subsections we fix a simple root with $\chi\left(x_{-\alpha}\right)=0$ and consider $I$ as in C.11(1). Note that

$$
W_{I} \alpha \subset(\alpha+\mathbf{Z} I) \cap R \subset R^{+} .
$$

Pick for each $\beta \in W_{I} \alpha$ an element $w_{\beta} \in W_{I}$ with $w_{\beta}^{-1} \alpha=\beta$ and set

$$
M^{\beta}=\operatorname{sbm}\left(s_{\alpha} w_{\beta}, \lambda\right) \text {. }
$$

Lemma C.11.b implies that

$$
\operatorname{dim} M^{\beta}=\left(p-\left\langle\lambda+\rho, \beta^{\vee}\right\rangle\right) p^{N-1}
$$

and that

$$
M^{\beta} \simeq Z_{\chi}\left(s_{\alpha} w_{\beta} \cdot \lambda, \alpha\right) .
$$

Claim. The submodule $M^{\beta}$ of $Z_{\chi}(\lambda)$ depends only on $\beta$, not the choice of $w_{\beta}$.

Proof. If $w_{\beta}^{\prime}$ is a second element in $W_{I}$ with $\left(w_{\beta}^{\prime}\right)^{-1} \alpha=\beta$, then $w=w_{\beta}^{\prime} w_{\beta}^{-1} \in W_{I}$ satisfies $w \alpha=\alpha$, hence $w s_{\alpha}=s_{\alpha} w$. It follows that $s_{\alpha} w_{\beta}^{\prime}=w s_{\alpha} w_{\beta} \in W_{I} s_{\alpha} w_{\beta}$; now apply Lemma C.11.a.

C.13. Lemma. Let $\gamma$ be a simple root, let $w \in W$ with $w \gamma \in W_{I} \alpha$. Then $\operatorname{sbm}(w, \lambda) / \operatorname{sbm}\left(w s_{\gamma}, \lambda\right)$ is a homomorphic image of $Z_{\chi}\left(w_{w \gamma} w_{\bullet} \lambda, \alpha\right)$.

Proof. The element $x=w_{w \gamma} w$ satisfies $x \gamma=\alpha$, hence $x s_{\gamma}=s_{\alpha} x$. Since $w_{w \gamma} \in W_{I}$, Lemma C.11.a implies that

$$
\operatorname{sbm}(w, \lambda)=\operatorname{sbm}(x, \lambda)
$$

and

$$
\operatorname{sbm}\left(w s_{\gamma}, \lambda\right)=\operatorname{sbm}\left(x s_{\gamma}, \lambda\right)=\operatorname{sbm}\left(s_{\alpha} x, \lambda\right) .
$$

Now the claim follows from Lemma C.7.c.

C.14. Proposition. Let $\beta \in W_{I} \alpha$ and $\gamma \in I$ with $\left\langle\gamma, \beta^{\vee}\right\rangle<0$.

a) We have

$$
M^{\beta} \supset M^{s_{\gamma} \beta}
$$

and

$$
\operatorname{dim}\left(M^{\beta} / M^{s_{\gamma} \beta}\right)=\left|\left\langle\gamma, \beta^{\vee}\right\rangle\right|\left\langle\lambda+\rho, \gamma^{\vee}\right\rangle p^{N-1} .
$$

b) Assume that $\left\langle\gamma, \beta^{\vee}\right\rangle=\left\langle\beta, \gamma^{\vee}\right\rangle=-1$. Then $\beta^{\prime}=w_{\beta}(\beta+\gamma)$ is a root and belongs to $W_{I} \alpha$; the element $x=w_{\beta^{\prime}} s_{\alpha} w_{\beta} \in W$ satisfies $x^{-1} \alpha=\gamma$ and

$$
M^{\beta} / M^{\beta+\gamma} \simeq Z_{\chi}(x \bullet \lambda, \alpha) \quad \text { if }\left\langle\lambda+\rho, \gamma^{\vee}\right\rangle>0 .
$$


Proof. a) We have clearly $s_{\gamma} \beta \in W_{I} \alpha$ and could choose $w_{s_{\gamma} \beta}=w_{\beta} s_{\gamma}$. So we have

$$
M^{s_{\gamma} \beta}=\operatorname{sbm}\left(s_{\alpha} w_{\beta} s_{\gamma}, \lambda\right) .
$$

On the other hand $s_{\beta} \gamma=\gamma-\left\langle\gamma, \beta^{\vee}\right\rangle \beta$ is a positive root not in $\mathbf{Z} I$. Therefore also $\beta^{\prime}=w_{\beta} s_{\beta} \gamma$ is positive. Using $s_{\beta}=w_{\beta}^{-1} s_{\alpha} w_{\beta}$, we get $\beta^{\prime}=s_{\alpha} w_{\beta} \gamma$, hence

$$
s_{\beta^{\prime}} s_{\alpha} w_{\beta}=\left(s_{\alpha} w_{\beta} s_{\gamma} w_{\beta}^{-1} s_{\alpha}\right) s_{\alpha} w_{\beta}=s_{\alpha} w_{\beta} s_{\gamma} .
$$

Since $\left(s_{\alpha} w_{\beta}\right)^{-1} \beta^{\prime}=\gamma>0$, we get from Proposition C.8 that

$$
\operatorname{sbm}\left(s_{\beta^{\prime}} s_{\alpha} w_{\beta}, \lambda\right) \subset \operatorname{sbm}\left(s_{\alpha} w_{\beta}, \lambda\right),
$$

hence (1). Now (2) follows from C.12(3) and $\left(s_{\gamma} \beta\right)^{\vee}=\beta^{\vee}-\left\langle\gamma, \beta^{\vee}\right\rangle \gamma^{\vee}$.

b) Now our assumptions imply that $s_{\gamma} \beta=\beta+\gamma=s_{\beta} \gamma$. It follows that $\beta+$ $\gamma \in W_{I} \alpha$ and that $\beta^{\prime}=w_{\beta} s_{\beta} \gamma=w_{\beta}(\beta+\gamma) \in W_{I} \alpha$. We observed above that $\beta^{\prime}=s_{\alpha} w_{\beta} \gamma$; so we can apply Lemma C.13 with $w=s_{\alpha} w_{\beta}$. Now $x=w_{\beta^{\prime}} s_{\alpha} w_{\beta}$ is the same $x$ as in the proof of Lemma C.13 and satisfies $\alpha=x \gamma$. We have

$$
M^{\beta}=\operatorname{sbm}(w, \lambda) \quad \text { and } \quad M^{\beta+\gamma}=\operatorname{sbm}\left(w s_{\gamma}, \lambda\right)
$$

since $w_{\beta} s_{\gamma}$ is a possible $w_{s_{\gamma} \beta}=w_{\beta+\gamma}$. Now Lemma C.13 says that we have a surjective homomorphism

$$
Z_{\chi}(x \cdot \lambda, \alpha) \longrightarrow M^{\beta} / M^{\beta+\gamma} .
$$

The left hand side has dimension equal to

$$
\left\langle x(\lambda+\rho), \alpha^{\vee}\right\rangle p^{N-1}=\left\langle\lambda+\rho, \gamma^{\vee}\right\rangle p^{N-1}
$$

as long as $\left\langle\lambda+\rho, \gamma^{\vee}\right\rangle>0$; otherwise its dimension is equal to $p^{N}$. In the first case, the surjection in (4) has to be an isomorphism by dimension comparison; this implies (3).

Remark. In the situation from b) one can deduce the inclusion in (1) directly from Lemma C.7.a (without the more complicated argument in C.8) using $x^{-1} \alpha=\gamma>0$ and the equalities

$$
M^{\beta}=\operatorname{sbm}(x, \lambda) \quad \text { and } \quad M^{\beta+\gamma}=\operatorname{sbm}\left(s_{\alpha} x, \lambda\right) .
$$

D.

We keep the assumptions from Sections B and C. We assume in addition:

$$
\text { The prime } p \text { is good for } R \text {. }
$$

and

$$
G \text { is almost simple. }
$$

The first assumption is crucial so that we can apply the Kac-Weisfeiler conjecture proved by Premet. The second assumption is mainly meant to simplify the statement of the results. 
D.1. We call a linear form $\chi \in \mathfrak{g}^{*}$ subregular if its orbit under the coadjoint action has dimension equal to $2(N-1)$ where $N=\left|R^{+}\right|$. If so, then the Kac-Weisfeiler conjecture as proved by Premet says: If $M$ is a $U_{\chi}(\mathfrak{g})$-module, then $\operatorname{dim}(M)$ is divisible by $p^{N-1}$.

Recall that we set $\mathfrak{p}_{\alpha}=\mathfrak{b}^{+}+\mathfrak{g}_{-\alpha}$ for each simple root $\alpha$; we denote (as in B.14) by $P_{\alpha}$ the corresponding parabolic subgroup of $G$. The following result is a translation of well known results on orbits in $\mathfrak{g}$ :

Lemma. There exists a unique subregular nilpotent orbit $\mathcal{O}$ in $\mathfrak{g}^{*}$. If $\alpha$ is a simple root, then $\mathcal{O}$ intersects the set of all $\chi \in \mathfrak{g}^{*}$ with $\chi\left(\mathfrak{p}_{\alpha}\right)=0$ in an open and dense subset. This intersection is one orbit under $P_{\alpha}$.

Proof. We can (under our assumptions on $p$ ) identify $\mathfrak{g}$ and $\mathfrak{g}^{*}$ as $G$-modules. The classification of the nilpotent orbits in $\mathfrak{g}$ is the same as for the Lie algebra over $\mathbf{C}$ of the same type (since $p$ is good). In particular, there is exactly one subregular nilpotent orbit in $\mathfrak{g}$; this yields the first claim.

The elements $\chi \in \mathfrak{g}^{*}$ with $\chi\left(\mathfrak{b}^{+}\right)=0$ and $\chi\left(x_{-\alpha}\right)=0$ correspond (under $\mathfrak{g} \simeq \mathfrak{g}^{*}$ ) to the elements in the nilradical $\mathfrak{n}^{\alpha}=\bigoplus_{\gamma>0, \gamma \neq \alpha} \mathfrak{g}_{\gamma}$ of the parabolic subalgebra $\mathfrak{p}_{\alpha}$. The theory of the Richardson orbits (cf. [C], 5.2.3) says that there exists exactly one nilpotent orbit for $G$ that intersects $\mathfrak{n}^{\alpha}$ in an open and dense subset. That intersection is one orbit under $P_{\alpha}$; furthermore the dimension of the orbit (under $G$ ) is equal to the codimension in $\mathfrak{g}$ of a Levi factor of $\mathfrak{p}_{\alpha}$. Since that codimension is equal to $2(N-1)$, we get the remaining claims.

Remark. For each simple root $\beta \neq \alpha$ the set of all $\chi$ with $\chi\left(\mathfrak{p}_{\alpha}\right)=0$ and $\chi\left(x_{-\beta}\right) \neq 0$ is an open and dense subset of the set of all $\chi$ with $\chi\left(\mathfrak{p}_{\alpha}\right)=0$. It follows: The set of all subregular $\chi \in \mathfrak{g}^{*}$ with $\chi\left(\mathfrak{p}_{\alpha}\right)=0$ and $\chi\left(x_{-\beta}\right) \neq 0$ for all simple roots $\beta \neq \alpha$ is an open and dense subset of the set of all $\chi$ with $\chi\left(\mathfrak{p}_{\alpha}\right)=0$.

D.2. Each subset $J$ of the set of all simple roots defines a facet $F(J)$ contained in $C_{0}^{\prime}$ as follows: A weight $\lambda \in C_{0}^{\prime}$ belongs to $F(J)$ if and only if $\left\langle\lambda+\rho, \beta^{\vee}\right\rangle>0$ for all $\beta \in J$ and $\left\langle\lambda+\rho, \gamma^{\vee}\right\rangle=0$ for all simple roots $\gamma \notin J$. Write $\varpi_{\gamma}$ for the fundamental weight corresponding to a simple root $\gamma$. Then $\lambda \in X$ belongs to $F(J)$ if there are integers $m_{\beta}>0$ such that $\lambda+\rho=\sum_{\beta \in J} m_{\beta} \varpi_{\beta}$ and if $\left\langle\lambda+\rho, \alpha^{\vee}\right\rangle<p$ for all $\alpha \in R^{+}$. Each facet (with respect to $W_{p}$ ) that is contained in $C_{0}^{\prime}$ has the form $F(J)$.

Lemma. Let $J$ be as above and let $\gamma \in J$. Suppose that we have for all $\lambda \in F(J)$ a module $N(\lambda)$ in $\mathcal{C}_{\lambda}$ with $\operatorname{dim} N(\lambda)=\left\langle\lambda+\rho, \gamma^{\vee}\right\rangle p^{N-1}$ such that $T_{\lambda}^{\mu} N(\lambda) \simeq N(\mu)$ for all $\lambda, \mu \in F(J)$. If $\chi$ is subregular, then each $N(\lambda)$ with $\lambda \in F(J)$ is simple.

Proof. We may assume that $F(J) \neq \emptyset$. Then the weight $\mu=-\rho+\sum_{\beta \in J} \varpi_{\beta}$ belongs to $F(J)$ since $\left\langle\mu+\rho, \alpha^{\vee}\right\rangle \leq\left\langle\lambda+\rho, \alpha^{\vee}\right\rangle\left\langle p\right.$ for all $\lambda \in F(J)$ and all $\alpha \in R^{+}$.

We have now $\operatorname{dim} N(\mu)=p^{N-1}$. Therefore Premet's theorem implies that $N(\mu)$ is simple. Proposition B.5 yields now simplicity in general.

Remark. Let $m$ be a positive integer. Suppose that we have in the lemma $\operatorname{dim} N(\lambda)$ $=m\left\langle\lambda+\rho, \gamma^{\vee}\right\rangle p^{N-1}$, while all the remaining assumptions are unchanged. Then the same proof as above yields that the length of $N(\lambda)$ is at most equal to $m$.

D.3. Here are two types of situations where we shall apply Lemma D.2. Let $\alpha$ be a simple root and let $\chi$ be subregular nilpotent with $\chi\left(\mathfrak{p}_{\alpha}\right)=0$. 
Lemma. a) Let $w \in W$ such that $w^{-1} \alpha$ is a simple root. Then $Z_{\chi}(w \cdot \lambda, \alpha)$ is simple for all $\lambda \in C_{0}^{\prime}$ with $\left\langle w(\lambda+\rho), \alpha^{\vee}\right\rangle>0$.

b) Let $\gamma$ be a simple root, let $w_{1}, w_{2} \in W$ such that $\operatorname{sbm}\left(w_{1}, \lambda\right) \subset \operatorname{sbm}\left(w_{2}, \lambda\right)$ and

$$
\operatorname{dim} \operatorname{sbm}\left(w_{2}, \lambda\right) / \operatorname{sbm}\left(w_{1}, \lambda\right)=\left\langle\lambda+\rho, \gamma^{\vee}\right\rangle p^{N-1}
$$

for all $\lambda \in C_{0}^{\prime}$. Then $\operatorname{sbm}\left(w_{2}, \lambda\right) / \operatorname{sbm}\left(w_{1}, \lambda\right)$ is simple for all $\lambda \in C_{0}^{\prime}$ with $\left\langle\lambda+\rho, \gamma^{\vee}\right\rangle>0$.

Proof. a) Let $J$ be the set of simple roots with $\lambda \in F(J)$. Then $\left\langle w(\lambda+\rho), \alpha^{\vee}\right\rangle>0$ is equivalent to $w^{-1} \alpha \in J$. If so, then

$$
\operatorname{dim} Z_{\chi}(w \cdot \lambda, \alpha)=\left\langle w(\lambda+\rho), \alpha^{\vee}\right\rangle p^{N-1}=\left\langle\lambda+\rho, w^{-1} \alpha^{\vee}\right\rangle p^{N-1} .
$$

Now the claim follows from Corollary B.11 and Lemma D.2.

b) This follows from C.9(1) and Lemma D.2. (Note that C.14(2) provides us with cases where our assumption is satisfied.)

D.4. Let $\alpha_{0}$ denote the unique short root that is a dominant weight. Set $h=$ $\left\langle\rho, \alpha_{0}^{\vee}\right\rangle+1$ equal to the Coxeter number of $R$.

Proposition. If $R$ is of type $E_{8}, F_{4}$, or $G_{2}$ suppose that $p>h+1$. Let $\alpha$ be a simple root and let $\chi$ be subregular nilpotent with $\chi\left(\mathfrak{p}_{\alpha}\right)=0$. Let $\lambda \in C_{0}^{\prime}$.

a) Let $w \in W$ with $w^{-1} \alpha=-\alpha_{0}$. Then $Z_{\chi}(w \bullet \lambda, \alpha)$ is simple and isomorphic to the socle of $Z_{\chi}(\lambda)$. We have $\operatorname{sbm}(w, \lambda)=\operatorname{soc} Z_{\chi}(\lambda)$.

b) The socle of $Z_{\chi}(\lambda)$ has dimension $\left(p-\left\langle\lambda+\rho, \alpha_{0}^{\vee}\right\rangle\right) p^{N-1}$.

Proof. a1) Suppose first that $R$ is not type $E_{8}, F_{4}$, or $G_{2}$. Under this assumption there exists a fundamental weight $\varpi$ with $\left\langle\varpi, \alpha_{0}^{\vee}\right\rangle=1$. Set

$$
\mu=\lambda+\left(p-1-\left\langle\lambda+\rho, \alpha_{0}^{\vee}\right\rangle\right) \varpi .
$$

We have then $\left\langle\mu+\rho, \alpha_{0}^{\vee}\right\rangle=p-1$; furthermore $\mu$ belongs to $C_{0}^{\prime}$ and $\lambda$ is in the closure of the facet of $\mu$. We have

$$
\left\langle w \cdot \mu, \alpha^{\vee}\right\rangle=\left\langle\mu+\rho, w^{-1} \alpha^{\vee}\right\rangle-1=-p \equiv 0 \quad(\bmod p),
$$

hence $\operatorname{dim} Z_{\chi}(w \bullet \mu, \alpha)=p^{N-1}$. Therefore $Z_{\chi}(w \bullet \mu, \alpha)$ is simple by Premet's theorem.

We have

$$
-p<-\left\langle\mu+\rho, \alpha_{0}^{\vee}\right\rangle=\left\langle w(\mu+\rho), \alpha^{\vee}\right\rangle \leq 0=\left\langle w \bullet(-\rho)+\rho, \alpha^{\vee}\right\rangle,
$$

hence by B.11

$$
T_{\mu}^{-\rho} Z_{\chi}(w \bullet \mu, \alpha) \simeq Z_{\chi}(w \cdot(-\rho), \alpha)=Z_{\chi}(-\rho) \neq 0 .
$$

So Proposition C.4.a implies that $Z_{\chi}(w \bullet \mu, \alpha)$ is isomorphic to the socle of $Z_{\chi}(\mu)$, hence $Z_{\chi}(w \bullet \mu, \alpha) \simeq \operatorname{sbm}\left(w_{0}, \mu\right)$ by C.10(2). Applying $T_{\mu}^{\lambda}$ we get [using B.11 and C.9(1)]

$$
Z_{\chi}(w \bullet \lambda, \alpha) \simeq \operatorname{sbm}\left(w_{0}, \lambda\right)=\operatorname{soc} Z_{\chi}(\lambda) .
$$

Note that $\operatorname{sbm}(w, \lambda)$ is a (non-zero) homomorphic image of $Z_{\chi}(w \cdot \lambda, \alpha)$ [by Lemma C.7.c and C.10(1)]. Because the latter module is simple, so is $\operatorname{sbm}(w, \lambda)$. Therefore it is equal to the (simple) socle of $Z_{\chi}(\lambda)$. 
a2) Consider now the case where $R$ is of type $E_{8}, F_{4}$, or $G_{2}$. Let $J$ be the set of simple roots with $\lambda \in F(J)$. We would like to find a weight $\mu \in C_{0}^{\prime}$ with $\left\langle\mu+\rho, \alpha_{0}^{\vee}\right\rangle=p-1$ such that $\lambda$ is in the closure of the facet of $\mu$. If so, then we can argue as above.

There exist fundamental weights $\varpi$ and $\varpi^{\prime}$ with $\left\langle\varpi, \alpha_{0}^{\vee}\right\rangle=2$ and $\left\langle\varpi^{\prime}, \alpha_{0}^{\vee}\right\rangle=3$. If $\left\langle\lambda+\rho, \alpha_{0}^{\vee}\right\rangle=p-1$, then we take $\mu=\lambda$ and we are done. Otherwise we try to find integers $r, s \geq 0$ such that

$$
\mu=r \varpi+s \varpi^{\prime}+\sum_{\beta \in J} \varpi_{\beta}-\rho
$$

satisfies $\left\langle\mu+\rho, \alpha_{0}^{\vee}\right\rangle=p-1$. This is possible unless $\left\langle\sum_{\beta \in J} \varpi_{\beta}, \alpha_{0}^{\vee}\right\rangle=p-2$. This equality can hold only when $p-2 \leq h-1$ since

$$
\sum_{\beta \in J}\left\langle\varpi_{\beta}, \alpha_{0}^{\vee}\right\rangle \leq\left\langle\rho, \alpha_{0}^{\vee}\right\rangle=h-1
$$

So our assumption $p>h+1$ makes sure that the "bad case" will not occur.

b) Let $L$ denote the socle of $Z_{\chi}(\lambda)$. Suppose first that $\alpha$ is short. Then $\alpha$ and $-\alpha_{0}$ are roots of the same length; so there exists $w \in W$ with $w\left(-\alpha_{0}\right)=\alpha$. Now a) implies that $L \simeq Z_{\chi}(w \bullet \lambda, \alpha)$. Since $p-\left\langle\lambda+\rho, \alpha_{0}^{\vee}\right\rangle \equiv\left\langle w(\lambda+\rho), \alpha^{\vee}\right\rangle(\bmod p)$ and $0<p-\left\langle\lambda+\rho, \alpha_{0}^{\vee}\right\rangle \leq p$, we get the claim from the formula in B.11.

Suppose now that $R$ has two root lengths and that $\alpha$ is long. Let then $\alpha^{\prime}$ be an arbitrary short simple root. We can find (see D.1) subregular $\chi^{\prime} \in \mathfrak{g}^{*}$ with $\chi^{\prime}\left(\mathfrak{b}^{+}\right)=0$ and $\chi^{\prime}\left(x_{-\alpha^{\prime}}\right)=0$. Since there exists only one subregular nilpotent orbit in $\mathfrak{g}^{*}$, there exists $g \in G$ with $g \chi=\chi^{\prime}$.

Let us use notations from B.13 like $\mathcal{C}_{\lambda}^{\prime}$ for $g \chi=\chi^{\prime}$. Applying the results so far to $\chi^{\prime}$ and $\alpha^{\prime}$ (instead of $\chi$ and $\alpha$ ) we see that there is a simple $U_{\chi^{\prime}}(\mathfrak{g})$-module $L_{1}$ in $\mathcal{C}_{\lambda}^{\prime}$ of dimension equal to $\left(p-\left\langle\lambda+\rho, \alpha_{0}^{\vee}\right\rangle\right) p^{N-1}$ and satisfying $\left(T_{\lambda}^{-\rho}\right)^{\prime} L_{1} \neq 0$. Twisting $L_{1}$ with $g^{-1}$ we get a simple module $L_{2}$ in $\mathcal{C}_{\lambda}$ of the same dimension with $T_{\lambda}^{-\rho} L_{2} \neq 0$ (by B.13(2)). Now C.4.a implies that $L_{2} \simeq L$; the claim follows.

Remarks. 1) The proof in a2) shows that the claim holds also in many cases where $R$ is of type $E_{8}, F_{4}$, and $G_{2}$ and $p \leq h+1$. One may hope that it holds always in good characteristic.

2) We have here deduced b) from a). Note conversely: If we know that b) holds (for a specific $\lambda$ ), then also a) holds (for that $\lambda$ ): We have $T_{\lambda}^{-\rho} Z_{\chi}(w \cdot \lambda, \alpha) \simeq Z_{\chi}(-\rho)$ for $w$ as in a) [by B.11]. Therefore (and by C.4) the socle of $Z_{\chi}(\lambda)$ is a composition factor of $Z_{\chi}\left(w_{\bullet} \lambda, \alpha\right)$. Now a dimension comparison shows that $Z_{\chi}(w \bullet \lambda, \alpha)$ is isomorphic to that socle, hence simple. The remainder of a) now follows as above.

D.5. Given $\lambda \in C_{0}^{\prime}$ write $J(\lambda)$ for the set of simple roots with $\lambda \in F(J(\lambda))$. Note that each $-\rho+\varpi_{\beta}$ with $\beta \in J(\lambda)$ is in the closure of the facet of $\lambda$. Let $\chi \in \mathfrak{g}^{*}$ be nilpotent. We associate to each module $M$ in $\mathcal{C}_{\lambda}$ an invariant by setting

$$
\kappa(M)=\left\{\beta \in J(\lambda) \cup\{0\} \mid T_{\lambda}^{\varpi \beta}-\rho(M) \neq 0\right\}
$$

where we use the convention $\varpi_{0}=0$.

This invariant will turn out to be useful in the case where $\chi$ is subregular. In the general case one should have a finer invariant that keeps track of the behaviour under all translations to the boundary of a given facet.

There may exist weights $\lambda, \lambda^{\prime} \in C_{0}^{\prime}$ with $\lambda \neq \lambda^{\prime}$ and $\mathcal{C}_{\lambda}=\mathcal{C}_{\lambda^{\prime}}$. If we replace $\lambda$ by $\lambda^{\prime}$ in (1), then we will in general get different results. So $\kappa$ depends not just on 
the category $\mathcal{C}_{\lambda}$, but also on the choice of $\lambda$. It might therefore be better to denote this map by $\kappa_{\lambda}$. However, usually we fix $\lambda$ and then no problems should arise.

Suppose that $\chi\left(\mathfrak{b}^{+}\right)=0$. Then C.9(1) and C.10(1) show that

$$
\kappa \operatorname{sbm}(w, \lambda)=J(\lambda) \cup\{0\} \quad \text { for all } w \in W .
$$

In particular, the socle $L=\operatorname{sbm}\left(w_{0}, \lambda\right)$ of $Z_{\chi}(\lambda)$ satisfies $\kappa(L)=J(\lambda) \cup\{0\}$. Proposition C. 4 says that this is the only simple module in $\mathcal{C}_{\lambda}$ with $0 \in \kappa(L)$.

Let $g \in G$. Given $M$ in $\mathcal{C}_{\lambda}$ we have ${ }^{g} M$ in $\mathcal{C}(g \chi)_{\lambda}$, see B.13. We get now

$$
\kappa\left({ }^{g} M\right)=\kappa(M)
$$

from B.13(2).

Lemma. Let $\chi \in \mathfrak{g}^{*}$ with $\chi\left(\mathfrak{b}^{+}\right)=0$.

a) Let $\gamma$ be a simple root and $w \in W$ with $w \gamma>0$. Then

$$
\kappa\left(\operatorname{sbm}(w, \lambda) / \operatorname{sbm}\left(w s_{\gamma}, \lambda\right)\right) \subset\{\gamma\} .
$$

b) If $L$ is a composition factor of $Z_{\chi}(\lambda)$ not isomorphic to $\operatorname{soc} Z_{\chi}(\lambda)$, then $\kappa(L)$ is either empty or consists of just one simple root.

c) Suppose that $\alpha$ is a simple root with $\chi\left(\mathfrak{p}_{\alpha}\right)=0$. Let $w \in W$ with $w^{-1} \alpha \in J(\lambda)$. Then $\kappa Z_{\chi}(w \bullet \lambda, \alpha)=\left\{w^{-1} \alpha\right\}$.

Proof. a) We have for all $\beta \in J(\lambda) \cup\{0\}$ by C.9(1)

$$
T_{\lambda}^{\varpi_{\beta}-\rho} \operatorname{sbm}(w, \lambda) / \operatorname{sbm}\left(w s_{\gamma}, \lambda\right) \simeq \operatorname{sbm}\left(w, \varpi_{\beta}-\rho\right) / \operatorname{sbm}\left(w s_{\gamma}, \varpi_{\beta}-\rho\right) .
$$

If $\beta \neq \gamma$, then we have $s_{\gamma} \bullet\left(\varpi_{\beta}-\rho\right)=\varpi_{\beta}-\rho$, hence $\operatorname{sbm}\left(w, \varpi_{\beta}-\rho\right)=\operatorname{sbm}\left(w s_{\gamma}\right.$, $\varpi_{\beta}-\rho$ ). So the right hand side in (4) is 0 in that case; the claim follows.

b) Let $w_{0}=s_{1} s_{2} \ldots s_{N}$ be a reduced decomposition of $w_{0}$. (So $s_{i}=s_{\gamma_{i}}$ for some simple root $\gamma_{i}$.) This leads to a chain of submodules

$$
Z_{\chi}(\lambda)=\operatorname{sbm}(1, \lambda) \supset \operatorname{sbm}\left(s_{1}, \lambda\right) \supset \operatorname{sbm}\left(s_{1} s_{2}, \lambda\right) \supset \cdots \supset \operatorname{sbm}\left(w_{0}, \lambda\right) .
$$

It follows that $L$ is a composition factor of $\operatorname{some} \operatorname{sbm}(w, \lambda) / \operatorname{sbm}\left(w s_{\gamma}, \lambda\right)$ with $w \in$ $W$ and $\gamma$ a simple root with $w \gamma>0$. Now the claim follows from a) since quite generally $\kappa\left(M^{\prime}\right) \subset \kappa(M)$ for any subquotient $M^{\prime}$ of a module $M$ in $\mathcal{C}_{\lambda}$.

c) Set $\beta=w^{-1} \alpha$. The definition of $J(\lambda)$ and of $C_{0}^{\prime}$ implies that $\mu=\lambda$ satisfies $0<\left\langle w \bullet \mu+\rho, \alpha^{\vee}\right\rangle<p$. The same inequalities hold for $\mu=\varpi_{\beta}-\rho$. On the other hand, all $\mu=\varpi_{\gamma}-\rho$ with $\gamma \neq \beta$ satisfy $\left\langle w \bullet \mu+\rho, \alpha^{\vee}\right\rangle=0$. Now the claim follows from Corollary B.11.

Remark. Let $\gamma$ be a simple root and let $m$ be a positive integer. Suppose that we have $w_{1}, w_{2} \in W$ with $\operatorname{sbm}\left(w_{1}, \lambda\right) \subset \operatorname{sbm}\left(w_{2}, \lambda\right)$ and

$$
\operatorname{dim} \operatorname{sbm}\left(w_{2}, \lambda\right) / \operatorname{sbm}\left(w_{1}, \lambda\right)=m\left\langle\lambda+\rho, \gamma^{\vee}\right\rangle p^{N-1}
$$

for all $\lambda \in C_{0}^{\prime}$. If $\gamma \in J(\lambda)$, then C.9(1) shows that

$$
\kappa \operatorname{sbm}\left(w_{2}, \lambda\right) / \operatorname{sbm}\left(w_{1}, \lambda\right)=\{\gamma\} .
$$


D.6. We are now ready to state our first main result (to be proved in D.7-D.10). Let $m_{\beta}$ denote the positive integers with

$$
\alpha_{0}^{\vee}=\sum_{\beta} m_{\beta} \beta^{\vee}
$$

where we sum over all simple roots $\beta$.

Theorem. Assume that $\chi$ is subregular nilpotent with $\chi\left(\mathfrak{b}^{+}\right)=0$. Exclude the case where $R$ is of type $G_{2}$; if $R$ is of type $E_{8}$ or $F_{4}$, assume that $p>h+1$. Let $\lambda \in C_{0}^{\prime}$. Then $Z_{\chi}(\lambda)$ has length $1+\sum_{\beta \in J(\lambda)} m_{\beta}$. We can denote the factors in a composition series of $Z_{\chi}(\lambda)$ by $L_{0}$ and $L_{\beta, i}$ with $\beta \in J(\lambda)$ and $1 \leq i \leq m_{\beta}$ such that

$$
\operatorname{dim} L_{\beta, i}=\left\langle\lambda+\rho, \beta^{\vee}\right\rangle p^{N-1} \quad \text { and } \quad \kappa\left(L_{\beta, i}\right)=\{\beta\}
$$

for all $\beta$ and $i$, while

$$
\operatorname{dim} L_{0}=\left(p-\left\langle\lambda+\rho, \alpha_{0}^{\vee}\right\rangle\right) p^{N-1} \quad \text { and } \quad \kappa\left(L_{0}\right)=J(\lambda) \cup\{0\} .
$$

Remarks. 1) The restrictions on the type are hoped to be unnecessary. If $R$ is of type $E_{8}$ or $F_{4}$ and $p \leq h+1$, then the theorem will hold for all $\lambda \in C_{0}^{\prime}$ where the socle of $Z_{\chi}(\lambda)$ has dimension equal to $\left(p-\left\langle\lambda+\rho, \alpha_{0}^{\vee}\right\rangle\right) p^{N-1}$. The same remark applies to the results in D.12 and D.13.

2) The theorem does not say whether different factors in the composition series are isomorphic to each other. If $L$ and $L^{\prime}$ are composition factors with $\kappa(L) \neq \kappa\left(L^{\prime}\right)$, then clearly $L \not L^{\prime}$. So the question is whether for fixed $\beta \in J(\lambda)$ the $m_{\beta}$ factors $L_{\beta, i}$ are isomorphic to each other. We shall see in Section $\mathrm{F}$ that such isomorphisms exist in certain cases.

3) The theorem confirms in part my speculations in [J3], 11.15 (where I look only at those $\lambda \in C_{0}^{\prime}$ that are $p$-regular, i.e., satisfy $\left\langle\lambda+\rho, \beta^{\vee}\right\rangle>0$ for all $\beta \in R^{+}$). The part of those speculations not confirmed is that the factors in a composition series should be pairwise non-isomorphic. As mentioned in the preceding remark, that turns out to be wrong.

D.7. We now begin to prove the theorem. Lemma B.13 and D.5(3) show: If Theorem D.6 holds for one subregular $\chi$, then it holds for all subregular $\chi$. We assume from now on that $\alpha$ is a simple root with $\chi\left(\mathfrak{p}_{\alpha}\right)=0$ and $\chi\left(x_{-\beta}\right) \neq 0$ for all simple roots $\beta \neq \alpha$. Remark D.1 shows that we can find $\chi$ with this property for each $\alpha$. Later on we shall make specific choices for $\alpha$. [Usually this is done such that the right hand side in (6) below is as small as possible.]

Set $L_{0}$ equal to the simple socle of $Z_{\chi}(\lambda)$. It satisfies D.6(3) by Proposition D.4.b and D.5(2).

By our choice of $\chi$ the set $I$ as in C.11(1) consists of all simple roots $\beta \neq$ $\alpha$. We shall use the construction from C.12-C.14 in order to find the remaining composition factors of $Z_{\chi}(\lambda)$.

We can construct inductively a chain

$$
\beta_{1}=\alpha, \beta_{2}, \ldots, \beta_{r}
$$

of roots in $W_{I} \alpha$ such that $\left\langle\beta_{r}, \gamma^{\vee}\right\rangle \geq 0$ for all $\gamma \in I$ and such that there exists for each $i<r$ a simple root $\gamma_{i} \in I$ with $\left\langle\beta_{i}, \gamma_{i}^{\vee}\right\rangle<0$ and $\beta_{i+1}=s_{\gamma_{i}} \beta_{i}$. 
The root $\beta_{r}$ is uniquely determined as the only weight in $W_{I} \alpha$ that is dominant with respect to the root system $R_{I}$ and its basis $I$. Set

$$
M_{i}=M^{\beta_{i}} \quad \text { for } 1 \leq i \leq r
$$

and $M_{0}=Z_{\chi}(\lambda)$. We have by C.14(1) a descending chain of submodules in $Z_{\chi}(\lambda)$

$$
M_{0}=Z_{\chi}(\lambda) \supset M_{1} \supset M_{2} \supset \cdots \supset M_{r} \supset \operatorname{sbm}\left(w_{0}, \lambda\right) \supset 0
$$

where the inclusion $M_{r} \supset \operatorname{sbm}\left(w_{0}, \lambda\right)$ follows from C.10(2). Furthermore, C.14(2) yields

$$
\operatorname{dim}\left(M_{i} / M_{i+1}\right)=\left|\left\langle\gamma_{i}, \beta_{i}^{\vee}\right\rangle\right|\left\langle\lambda+\rho, \gamma_{i}^{\vee}\right\rangle p^{N-1} \quad \text { for } 1 \leq i<r .
$$

On the other hand, $M_{1}=M^{\alpha}=\operatorname{sbm}\left(s_{\alpha}, \lambda\right)$ shows that $M_{0} / M_{1} \simeq Z_{\chi}(\lambda, \alpha)$ and

$$
\operatorname{dim}\left(M_{0} / M_{1}\right)=\left\langle\lambda+\rho, \alpha^{\vee}\right\rangle p^{N-1} .
$$

Finally, a comparison of C.12(4) and Proposition D.4.b shows that

$$
\operatorname{dim}\left(M_{r} / \operatorname{sbm}\left(w_{0}, \lambda\right)\right)=\left\langle\lambda+\rho, \alpha_{0}^{\vee}-\beta_{r}^{\vee}\right\rangle p^{N-1} .
$$

D.8. Suppose first that all roots in $R$ have the same length. We choose $\chi$ such that (in the notations from Bourbaki's tables in [Bou]) $\alpha=\alpha_{7}$ (resp. $\alpha=\alpha_{8}$ ) for $R$ of type $E_{7}$ (resp. of type $E_{8}$ ) and $\alpha=\alpha_{1}$ otherwise. Then one checks by inspection

$$
\beta_{r}= \begin{cases}\alpha_{0}-\alpha, & \text { for } R \text { of type } E_{8} ; \\ \alpha_{0}, & \text { otherwise. }\end{cases}
$$

Writing $\gamma_{0}=\alpha$ we get from D.7(4),(5)

$$
\operatorname{dim}\left(M_{i} / M_{i+1}\right)=\left\langle\lambda+\rho, \gamma_{i}^{\vee}\right\rangle p^{N-1} \quad \text { for } 0 \leq i<r
$$

since $\beta_{i}$ and $\gamma_{i}$ have the same length (for $i>0$ ).

If $\gamma_{i} \notin J(\lambda)$, then clearly $M_{i} / M_{i+1}=0$. If $\gamma_{i} \in J(\lambda)$, then Lemma D.3.b implies that $M_{i} / M_{i+1}$ is simple; furthermore D.5(5) shows that $\kappa\left(M_{i} / M_{i+1}\right)=\left\{\gamma_{i}\right\}$.

Exclude the case $E_{8}$ for the moment. Then (1) and D.7(6) show that $M_{r}$ is equal to the simple socle $L_{0}$ of $Z_{\chi}(\lambda)$. So the results in the preceding paragraph show that the $M_{i}$ provides us with a composition series of $Z_{\chi}(\lambda) / L_{0}$. The length of this series is equal to the number of $i$ with $0 \leq i<r$ and $\gamma_{i} \in J(\lambda)$. If $\beta \in J(\lambda)$ and if $m_{\beta}^{\prime}$ is the number of $i$ with $\gamma_{i}=\beta$, then the composition series has exactly $m_{\beta}^{\prime}$ factors $L$ with $\operatorname{dim}(L)=\left\langle\lambda+\rho, \beta^{\vee}\right\rangle p^{N-1}$ and $\kappa(L)=\{\beta\}$. We have $\beta_{i}=\beta_{i-1}+\gamma_{i-1}$ for $2 \leq i \leq r$ and $\beta_{1}=\alpha=\gamma_{0}$, hence

$$
\sum_{i=0}^{r-1} \gamma_{i}=\beta_{r}=\alpha_{0}=\sum_{\beta} m_{\beta} \beta .
$$

This shows that $m_{\beta}^{\prime}=m_{\beta}$; the claim follows.

If $R$ is of type $E_{8}$, then set $M_{r+1}=\operatorname{sbm}\left(w_{0}, \lambda\right)$ and $\gamma_{r}=\alpha$. Now (1) and D.7(6) yield

$$
\operatorname{dim}\left(M_{r} / M_{r+1}\right)=\left\langle\lambda+\rho, \gamma_{r}^{\vee}\right\rangle p^{N-1} .
$$

Again Lemma D.3.b and D.5(5) show that $M_{r} / M_{r+1}$ is simple with $\kappa\left(M_{r} / M_{r+1}\right)$ $=\left\{\gamma_{r}\right\}$. Now argue as in the preceding paragraph. 
D.9. Consider now $R$ of type $B_{n}$ or $C_{n}$ with $n \geq 2$. We choose $\chi$ such that $\alpha=\alpha_{1}$ (in the notations from [Bou]) and get then (in those notations) $W_{I} \alpha=\left\{\varepsilon_{1} \pm \varepsilon_{i} \mid\right.$ $2 \leq i \leq n\}$. The sequence from D.7(1) is now equal to

$$
\varepsilon_{1}-\varepsilon_{2}, \varepsilon_{1}-\varepsilon_{3}, \ldots, \varepsilon_{1}-\varepsilon_{n}, \varepsilon_{1}+\varepsilon_{n}, \ldots, \varepsilon_{1}+\varepsilon_{3}, \varepsilon_{1}+\varepsilon_{2} .
$$

We have $\gamma_{i}=\varepsilon_{i+1}-\varepsilon_{i+2}=\alpha_{i+1}$ for $1 \leq i<n-1$, and $\gamma_{n-1}=\alpha_{n}$ and $\gamma_{n+i}=$ $\varepsilon_{n-i-1}-\varepsilon_{n-i}=\alpha_{n-i-1}$ for $0 \leq i<n-2$. We get in particular that $r=2 n-2$ and that

$$
\beta_{r}^{\vee}=\left(\varepsilon_{1}+\varepsilon_{2}\right)^{\vee}= \begin{cases}\alpha_{0}^{\vee}-\alpha^{\vee}, & \text { for type } B_{n} ; \\ \alpha_{0}^{\vee}, & \text { for type } C_{n} .\end{cases}
$$

If $R$ is of type $C_{n}$, then D.8(6) implies now that $M_{r}$ is equal to the simple socle $L_{0}$ of $Z_{\chi}(\lambda)$. For $R$ of type $B_{n}$ we get instead

$$
\operatorname{dim}\left(M_{r} / \operatorname{sbm}\left(w_{0}, \lambda\right)\right)=\left\langle\lambda+\rho, \alpha^{\vee}\right\rangle p^{N-1} .
$$

Now Lemma D.3.b and D.5(5) show that $M_{r} / \operatorname{sbm}\left(w_{0}, \lambda\right)$ is simple with $\kappa$ invariant equal to $\{\alpha\}$ in case $\left\langle\lambda+\rho, \alpha^{\vee}\right\rangle>0$; otherwise this factor module is equal to 0 .

If $R$ is of type $B_{n}$, then $\alpha$ and hence all $\beta_{i}$ are long. We get therefore $\left\langle\gamma_{i}, \beta_{i}^{\vee}\right\rangle=$ -1 for $1 \leq i<r$, hence [using D.7(4),(5)]

$$
\operatorname{dim}\left(M_{i} / M_{i+1}\right)=\left\langle\lambda+\rho, \gamma_{i}^{\vee}\right\rangle p^{N-1} \quad \text { for } 0 \leq i<r
$$

setting $\gamma_{0}=\alpha$. One gets then (as in D.8) that all $M_{i} / M_{i+1}$ are simple or 0 , with $\kappa$ invariant equal to $\left\{\gamma_{i}\right\}$ in the first case. Then the theorem follows by counting the numbers of $i$ with $\gamma_{i}=\beta$ for each simple $\operatorname{root} \beta$.

Assume now that $R$ is of type $C_{n}$. Then (3) holds for all $i \neq n-1$ and we can argue as in the preceding paragraph for these factors. However, we now get

$$
\operatorname{dim}\left(M_{n-1} / M_{n}\right)=2\left\langle\lambda+\rho, \alpha_{n}^{\vee}\right\rangle p^{N-1} .
$$

If $\left\langle\lambda+\rho, \alpha_{n}^{\vee}\right\rangle=0$, then $M_{n-1}=M_{n}$ and we have already a composition series. Counting the numbers of $i$ with $\gamma_{i}=\beta$ then yields the claim. Suppose from now on that $\left\langle\lambda+\rho, \alpha_{n}^{\vee}\right\rangle>0$. We want to show in this case that $M_{n-1} / M_{n}$ has length 2 , with both composition factors of dimension $\left\langle\lambda+\rho, \alpha_{n}^{\vee}\right\rangle p^{N-1}$ and with $\kappa$ invariant equal to $\left\{\alpha_{n}\right\}$. Then the theorem will follow as before.

Formula (4) implies by Remark D.2 that $M_{n-1} / M_{n}$ has length at most equal to 2. Note next that $\mu=-\varpi_{n}+\rho$ is in the closure of the facet of $\lambda$; we have

$$
\operatorname{dim} T_{\lambda}^{\mu}\left(M_{n-1} / M_{n}\right)=2 p^{N-1} .
$$

We can find (by Lemma D.1) an element $g \in G$ such that $(g \chi)\left(\mathfrak{b}^{+}\right)=0$ and $(g \chi)\left(x_{-\alpha_{n}}\right) \neq 0$. Then $L=Z_{g \chi}\left(\lambda, \alpha_{n}\right)$ is (by Lemma D.3.a) a simple module of dimension equal to $\left\langle\lambda+\rho, \alpha_{n}^{\vee}\right\rangle p^{N-1}$; we have furthermore $\kappa(L)=\left\{\alpha_{n}\right\}$. More precisely, $T_{\lambda}^{\mu} L \simeq Z_{g \chi}\left(\mu, \alpha_{n}\right)$ has dimension equal to $p^{N-1}$. Then $L^{\prime}=g^{-1} L$ is a simple module in $\mathcal{C}_{\lambda}$ of the same dimension with $\kappa\left(L^{\prime}\right)=\left\{\alpha_{n}\right\}$ by B.13(2) and $\operatorname{dim} T_{\lambda}^{\mu} L^{\prime}=p^{N-1}$.

Since $L^{\prime}$ is in $\mathcal{C}_{\lambda}$ it has to be a composition factor of $Z_{\chi}(\lambda)$. All $M_{i} / M_{i+1}$ with $i \neq n-1$ and $M_{2 n-2}$ are simple with $\kappa$ invariant different from $\left\{\alpha_{n}\right\}$. Therefore $L^{\prime}$ has to be a composition factor of $M_{n-1} / M_{n}$. It follows that this factor module has length equal to 2 and that the second composition factor, say $L^{\prime \prime}$, has to have the same dimension. Furthermore, the exactness of the translation functors implies that $T_{\lambda}^{\mu}\left(M_{n-1} / M_{n}\right)$ has a filtration with factors $T_{\lambda}^{\mu} L^{\prime}$ and $T_{\lambda}^{\mu} L^{\prime \prime}$. By comparison of dimensions we get that also $T_{\lambda}^{\mu} L^{\prime \prime}$ has dimension equal to $p^{N-1}$. This shows that 
$\alpha_{n} \in \kappa\left(L^{\prime \prime}\right)$. On the other hand, since $L^{\prime \prime}$ is a composition factor of $M_{n-1} / M_{n}$ we have

$$
\kappa\left(L^{\prime \prime}\right) \subset \kappa\left(M_{n-1} / M_{n}\right)=\left\{\alpha_{n}\right\} .
$$

So we get equality: $\kappa\left(L^{\prime \prime}\right)=\left\{\alpha_{n}\right\}$. This completes the proof of the claim concerning the composition factors of $M_{n-1} / M_{n}$, hence that of the theorem for type $C_{n}$.

D.10. Consider $R$ of type $F_{4}$. We choose $\chi$ such that $\alpha=\alpha_{4}$ in the notations from [Bou]. So $\alpha$ is short. We can choose the chain in D.7(1) such that the corresponding simple roots are (in this order)

$$
\alpha_{3}, \alpha_{2}, \alpha_{1}, \alpha_{3}, \alpha_{2}, \alpha_{3} .
$$

We get $r=7$ and $\beta_{7}=\alpha_{1}+2 \alpha_{2}+3 \alpha_{3}+\alpha_{4}$, hence

$$
\beta_{7}^{\vee}=2 \alpha_{1}^{\vee}+4 \alpha_{2}^{\vee}+3 \alpha_{3}^{\vee}+\alpha_{4}^{\vee}=\alpha_{0}^{\vee}-\alpha_{4}^{\vee} .
$$

Set $M_{8}=\operatorname{sbm}\left(w_{0}, \lambda\right)$. We get from D.7(4)-(6)

$$
\begin{aligned}
& \operatorname{dim}\left(M_{0} / M_{1}\right)=\operatorname{dim}\left(M_{7} / M_{8}\right)=\left\langle\lambda+\rho, \alpha_{4}^{\vee}\right\rangle p^{N-1}, \\
& \operatorname{dim}\left(M_{1} / M_{2}\right)=\operatorname{dim}\left(M_{4} / M_{5}\right)=\operatorname{dim}\left(M_{6} / M_{7}\right)=\left\langle\lambda+\rho, \alpha_{3}^{\vee}\right\rangle p^{N-1}, \\
& \operatorname{dim}\left(M_{2} / M_{3}\right)=\operatorname{dim}\left(M_{5} / M_{6}\right)=2\left\langle\lambda+\rho, \alpha_{2}^{\vee}\right\rangle p^{N-1}, \\
& \operatorname{dim}\left(M_{3} / M_{4}\right)=2\left\langle\lambda+\rho, \alpha_{1}^{\vee}\right\rangle p^{N-1} .
\end{aligned}
$$

Lemma D.3.b implies that $M_{0} / M_{1}, M_{7} / M_{8}, M_{1} / M_{2}, M_{4} / M_{5}$, and $M_{6} / M_{7}$ are simple or 0 ; if non-zero, then the first two modules have $\kappa$ invariant $\left\{\alpha_{4}\right\}$, and the remaining three modules have $\kappa$ invariant $\left\{\alpha_{3}\right\}$.

Each factor module $M_{2} / M_{3}, M_{5} / M_{6}, M_{3} / M_{4}$ has length at most equal to 2 by the Remark D.2. We have

$$
\kappa\left(M_{2} / M_{3}\right)=\kappa\left(M_{5} / M_{6}\right)=\left\{\alpha_{2}\right\} \quad \text { and } \quad \kappa\left(M_{3} / M_{4}\right)=\left\{\alpha_{1}\right\} .
$$

More precisely, we have, if $\alpha_{2} \in J(\lambda)$,

$$
\operatorname{dim} T_{\lambda}^{\varpi_{2}-\rho}\left(M_{2} / M_{3}\right)=\operatorname{dim} T_{\lambda}^{\varpi_{2}-\rho}\left(M_{5} / M_{6}\right)=2 p^{N-1}
$$

and, if $\alpha_{1} \in J(\lambda)$,

$$
\operatorname{dim} T_{\lambda}^{\varpi_{1}-\rho}\left(M_{3} / M_{4}\right)=2 p^{N-1} .
$$

The theorem will follow if we can show: If $\alpha_{1} \in J(\lambda)$, then $M_{3} / M_{4}$ has length 2 with both factors of dimension $\left\langle\lambda+\rho, \alpha_{1}^{\vee}\right\rangle p^{N-1}$ and with $\kappa$ invariant $\left\{\alpha_{1}\right\}$. If $\alpha_{2} \in J(\lambda)$, then both $M_{2} / M_{3}$ and $M_{5} / M_{6}$ have length 2 with all simple factors of dimension $\left\langle\lambda+\rho, \alpha_{2}^{\vee}\right\rangle p^{N-1}$ and with $\kappa$ invariant $\left\{\alpha_{2}\right\}$.

Set $\alpha^{\prime}=\alpha_{1}$. Choose $g \in G$ such that $\chi^{\prime}=g \chi$ satisfies $\chi^{\prime}\left(\mathfrak{b}^{+}\right)=0$ and $\chi^{\prime}\left(x_{-\alpha^{\prime}}\right)=$ 0 . (This is possible by D.1.) We can carry out the constructions from C.12C.14 and D.7 with $\chi^{\prime}$ and $\alpha^{\prime}$ instead of $\chi$ and $\alpha$. Let me write $\beta_{i}^{\prime}$ and $\gamma_{i}^{\prime}$ for the corresponding roots and $M_{i}^{\prime}$ for the corresponding submodules of $Z_{\chi^{\prime}}(\lambda)$. We can choose the sequence as in D.7(1) such that the sequence of the $\gamma_{i}^{\prime}$ is equal to

$$
\alpha_{2}, \alpha_{3}, \alpha_{4}, \alpha_{2}, \alpha_{3}, \alpha_{2}
$$


We get from D.7(4)-(6)

$$
\begin{aligned}
& \operatorname{dim}\left(M_{0}^{\prime} / M_{1}^{\prime}\right)=\left\langle\lambda+\rho, \alpha_{1}^{\vee}\right\rangle p^{N-1}, \\
& \operatorname{dim}\left(M_{1}^{\prime} / M_{2}^{\prime}\right)=\operatorname{dim}\left(M_{4}^{\prime} / M_{5}^{\prime}\right)=\operatorname{dim}\left(M_{6}^{\prime} / M_{7}^{\prime}\right)=\left\langle\lambda+\rho, \alpha_{2}^{\vee}\right\rangle p^{N-1}, \\
& \operatorname{dim}\left(M_{2}^{\prime} / M_{3}^{\prime}\right)=\operatorname{dim}\left(M_{5}^{\prime} / M_{6}^{\prime}\right)=\left\langle\lambda+\rho, \alpha_{3}^{\vee}\right\rangle p^{N-1}, \\
& \operatorname{dim}\left(M_{3}^{\prime} / M_{4}^{\prime}\right)=\left\langle\lambda+\rho, \alpha_{4}^{\vee}\right\rangle p^{N-1} .
\end{aligned}
$$

Lemma D.3.b implies that each $M_{i}^{\prime} / M_{i+1}^{\prime}$ with $0 \leq i<7$ is simple or 0 ; if $\operatorname{dim}\left(M_{i}^{\prime} / M_{i+1}^{\prime}\right)=\left\langle\lambda+\rho, \alpha_{j}^{\vee}\right\rangle p^{N-1}$ and $\alpha_{j} \in J(\lambda)$, then $M_{i}^{\prime} / M_{i+1}^{\prime}$ has $\kappa$ invariant $\left\{\alpha_{j}\right\}$ and satisfies $\operatorname{dim} T_{\lambda}^{\varpi_{j}-\rho}\left(M_{i}^{\prime} / M_{i+1}^{\prime}\right)=p^{N-1}$.

Twisting with $g^{-1}$ we get: If $\alpha_{1} \in J(\lambda)$, then $Z_{\chi}(\lambda)$ has a composition factor $L_{1,1}$ of dimension $\left\langle\lambda+\rho, \alpha_{1}^{\vee}\right\rangle p^{N-1}$, with $\kappa$ invariant $\left\{\alpha_{1}\right\}$ and $\operatorname{dim} T_{\lambda}^{\varpi_{1}-\rho}\left(L_{1,1}\right)=$ $p^{N-1}$. If $\alpha_{2} \in J(\lambda)$, then a composition series of $Z_{\chi}(\lambda)$ contains three quotients $L_{2,1}, L_{2,2}$, and $L_{2,3}$, all of dimension $\left\langle\lambda+\rho, \alpha_{2}^{\vee}\right\rangle p^{N-1}$, with $\kappa$ invariant $\left\{\alpha_{2}\right\}$ and $\operatorname{dim} T_{\lambda}^{\varpi_{2}-\rho}\left(L_{2, i}\right)=p^{N-1}$. (Here one has to use the full strength of Lemma B.13.)

A look at the $\kappa$ invariants of the $M_{i} / M_{i+1}$ and of $M_{8}$ shows now: If $\alpha_{1} \in J(\lambda)$, then $L_{1,1}$ is a composition factor of $M_{3} / M_{4}$. If $\alpha_{2} \in J(\lambda)$, then $L_{2,1}, L_{2,2}, L_{2,3}$ are factors in a composition series of $M_{2} / M_{3} \oplus M_{5} / M_{6}$. Now one concludes the proof arguing as in type $C_{n}$.

D.11. Consider $R$ of type $G_{2}$. We choose $\chi$ such that $\alpha=\alpha_{1}$ in the notations from [Bou]. So $\alpha$ is short. Assume that the socle of $Z_{\chi}(\lambda)$ has the expected dimension. When we carry out our standard construction we get $r=2$ and $\beta_{r}=\alpha_{1}+\alpha_{2}$, hence $\beta_{r}^{\vee}=\alpha_{1}^{\vee}+3 \alpha_{2}^{\vee}=\alpha_{0}^{\vee}-\alpha_{1}^{\vee}$. Setting $M_{3}=\operatorname{sbm}\left(w_{0}, \lambda\right)$ we get

$$
\begin{aligned}
& \operatorname{dim}\left(M_{0} / M_{1}\right)=\operatorname{dim}\left(M_{2} / M_{3}\right)=\left\langle\lambda+\rho, \alpha_{1}^{\vee}\right\rangle p^{N-1}, \\
& \operatorname{dim}\left(M_{1} / M_{2}\right)=3\left\langle\lambda+\rho, \alpha_{2}^{\vee}\right\rangle p^{N-1} .
\end{aligned}
$$

We see that $M_{0} / M_{1}$ and $M_{2} / M_{3}$ are simple or 0 , with $\kappa$ invariant $\left\{\alpha_{1}\right\}$ if non-zero. If $\left\langle\lambda+\rho, \alpha_{2}^{\vee}\right\rangle=0$, then we are done. So assume that $\left\langle\lambda+\rho, \alpha_{2}^{\vee}\right\rangle>0$. In order to extend Theorem D. 6 to type $G_{2}$, we would have to show that $M_{1} / M_{2}$ has length 3 with all factors of dimension $\left\langle\lambda+\rho, \alpha_{2}^{\vee}\right\rangle p^{N-1}$ and $\kappa$ invariant $\left\{\alpha_{2}\right\}$. The way that we handle such problems in types $C_{n}$ and $F_{4}$ was to look at another subregular $\chi^{\prime}$ and to get simple modules of the right type by twisting. If we do this here, we get one simple module $L$ that has to occur as a composition factor of $M_{1} / M_{2}$ having the expected dimension and the expected $\kappa$ invariant. But one such factor is not enough when the expected length is 3 .

D.12. Return to the situation from Theorem D.6. So we exclude the case where $R$ is of type $G_{2}$; if $R$ is of type $E_{8}$ or $F_{4}$, assume that $p>h+1$. If $R$ is of type $B_{n}$ set $\alpha=\alpha_{n}$; otherwise let $\alpha$ be the same simple root that was used in D.8-D.10. So $\alpha$ is a short simple root in all cases. Choose $\chi$ subregular with $\chi\left(\mathfrak{p}_{\alpha}\right)=0$. We claim under these assumptions:

Lemma. Let $\lambda \in C_{0}^{\prime}$. Let $\gamma$ be a short simple root with $\gamma \in J(\lambda)$. If $L$ is a simple module in $\mathcal{C}_{\lambda}$ with $\kappa(L)=\{\gamma\}$, then there exists an element $x \in W$ with $x \gamma=\alpha$ and $L \simeq Z_{\chi}(x \bullet \lambda, \alpha)$.

Proof. If the claim holds for one $\chi$ as above, then it holds also for all $g \chi$ with $g \in P_{\alpha}$; this follows easily from B.14(3) and D.5(3). This means by Lemma D.1 
that it suffices to prove the claim for one special $\chi$. We can therefore assume that $\chi\left(x_{-\beta}\right) \neq 0$ for all simple roots $\beta \neq \alpha$.

Suppose at first that $\gamma \neq \alpha$. (Note that this case does not occur for $R$ of type $B_{n}$.) Since $L$ is isomorphic to one of the $L_{\gamma, i}$ from Theorem D.6, the proofs in D.8-D.10 show that $L$ is isomorphic to one of the factors $M_{j} / M_{j+1}$ with $1<j<r$ in D.7(3). In these cases the claim follows from C.14(3).

So assume that $\gamma=\alpha$. If $m_{\alpha}=1$, then we have $L \simeq Z_{\chi}(\lambda, \alpha)$ and we can take $x=1$.

Assume from now on that $m_{\alpha}>1$. Then $R$ is of type $E_{8}$ or $F_{4}$; we have $m_{\alpha}=2$. The two composition factors $L_{\alpha, 1}$ and $L_{\alpha, 2}$ in D.6 arise as

$$
L_{\alpha, 1}=Z_{\chi}(\lambda) / M^{\alpha} \simeq Z_{\chi}(\lambda, \alpha)
$$

and

$$
L_{\alpha, 2}=M^{\alpha_{0}-\alpha} / \operatorname{soc} Z_{\chi}(\lambda) .
$$

We can take $x=1$ if $L \simeq L_{\alpha, 1}$. So we may assume that $L \simeq L_{\alpha, 2}$.

Recall that $\alpha=\alpha_{8}$ in type $E_{8}$ and $\alpha=\alpha_{4}$ in type $F_{4}$. In both cases $\alpha_{0}$ is the fundamental weight $\varpi_{\alpha}$ corresponding to $\alpha$.

Set $\alpha^{\prime}$ equal to the unique simple root with $\left(\alpha, \alpha^{\prime}\right)<0$. Then $\alpha^{\prime}$ is short; we have $s_{\alpha} \alpha^{\prime}=\alpha^{\prime}+\alpha=s_{\alpha^{\prime}} \alpha$.

Write $I$ (as in D.7) for the set of simple roots different from $\alpha$. Let $w_{I}$ be the longest element in $W_{I}$. It is an involution and satisfies

$$
w_{I} \alpha^{\prime}=-\alpha^{\prime} \quad \text { and } \quad w_{I} \alpha=\alpha_{0}-\alpha .
$$

Here the first equality follows from the fact that $I$ is of type $E_{7}$ or $B_{3}$, hence that $w_{I}$ acts as -1 on all of $I$. For the second equality note that $\alpha$ (resp. $\alpha_{0}-\alpha$ ) is the unique weight in $W_{I} \alpha$ that is antidominant (resp. dominant) with respect to $I$.

Claim. The element $w_{1}=s_{\alpha} w_{I} s_{\alpha^{\prime}}$ satisfies

$$
\operatorname{sbm}\left(w_{1}, \lambda\right) \supset \operatorname{sbm}\left(w_{1} s_{\alpha^{\prime}}, \lambda\right) \supset \operatorname{sbm}\left(w_{1} s_{\alpha^{\prime}} s_{\alpha}, \lambda\right) \supset \operatorname{sbm}\left(w_{1} s_{\alpha+\alpha^{\prime}}, \lambda\right)
$$

and

$$
\operatorname{sbm}\left(w_{1}, \lambda\right) \supset \operatorname{sbm}\left(w_{1} s_{\alpha}, \lambda\right) \supset \operatorname{sbm}\left(w_{1} s_{\alpha} s_{\alpha^{\prime}}, \lambda\right) \supset \operatorname{sbm}\left(w_{1} s_{\alpha+\alpha^{\prime}}, \lambda\right)
$$

and

$$
\operatorname{sbm}\left(w_{1} s_{\alpha^{\prime}}, \lambda\right) / \operatorname{sbm}\left(w_{1} s_{\alpha^{\prime}} s_{\alpha}, \lambda\right) \simeq L_{\alpha, 2} .
$$

Proof (of Claim). We have $s_{\alpha} \alpha_{0}=\alpha_{0}-\alpha$ and $s_{\alpha^{\prime}} \alpha_{0}=\alpha_{0}$, hence

$$
w_{1} \alpha=s_{\alpha} w_{I}\left(\alpha+\alpha^{\prime}\right)=s_{\alpha}\left(\alpha_{0}-\alpha-\alpha^{\prime}\right)=\alpha_{0}-\alpha-\alpha^{\prime}
$$

and

$$
w_{1} \alpha^{\prime}=s_{\alpha} w_{I}\left(-\alpha^{\prime}\right)=s_{\alpha} \alpha^{\prime}=\alpha+\alpha^{\prime} .
$$

Since both $w_{1} \alpha$ and $w_{1} \alpha^{\prime}$ are positive, we have in the Bruhat-Chevalley order

$$
w_{1}<w_{1} s_{\alpha^{\prime}}<w_{1} s_{\alpha^{\prime}} s_{\alpha}<w_{1} s_{\alpha^{\prime}} s_{\alpha} s_{\alpha^{\prime}}=w_{1} s_{\alpha+\alpha^{\prime}}
$$

and

$$
w_{1}<w_{1} s_{\alpha}<w_{1} s_{\alpha} s_{\alpha^{\prime}}<w_{1} s_{\alpha} s_{\alpha^{\prime}} s_{\alpha}=w_{1} s_{\alpha+\alpha^{\prime}} .
$$

This yields (4) and (5) using C.8(11). 
The second equality in (3) implies by C.12(2)

$$
\operatorname{sbm}\left(s_{\alpha} w_{I}, \lambda\right)=M^{\alpha_{0}-\alpha}
$$

We get also $s_{\alpha} w_{I} s_{\alpha}(\alpha)=-s_{\alpha}\left(\alpha_{0}-\alpha\right)=-\alpha_{0}$, hence by Proposition D.4.a

$$
\operatorname{sbm}\left(s_{\alpha} w_{I} s_{\alpha}, \lambda\right)=\operatorname{soc} Z_{\chi}(\lambda) .
$$

Using $w_{1} s_{\alpha^{\prime}}=s_{\alpha} w_{I}$ and $w_{1} s_{\alpha^{\prime}} s_{\alpha}=s_{\alpha} w_{I} s_{\alpha}$, we see that (6) follows from (2), (7), and (8). This concludes the proof of the claim.

Now (4)-(6) imply that $L_{\alpha, 2}$ is a composition factor of one of the subsequent quotients in (5). Because $\operatorname{sbm}\left(w_{1} s_{\alpha}, \lambda\right) / \operatorname{sbm}\left(w_{1} s_{\alpha} s_{\alpha^{\prime}}, \lambda\right)$ has $\kappa$ invariant contained in $\left\{\alpha^{\prime}\right\}$ by Lemma D.5.a, $L_{\alpha, 2}$ cannot be a composition factor of this quotient. So it is a composition factor of $\operatorname{sbm}\left(w_{2}, \lambda\right) / \operatorname{sbm}\left(w_{2} s_{\alpha}, \lambda\right)$ with $w_{2}=w_{1}$ or $w_{2}=$ $w_{1} s_{\alpha} s_{\alpha^{\prime}}$. We have $w_{1} \alpha=\alpha_{0}-\alpha-\alpha^{\prime}$ and $w_{1} s_{\alpha} s_{\alpha^{\prime}} \alpha=w_{1} \alpha^{\prime}=\alpha+\alpha^{\prime}$, hence $w_{2} \alpha \in W_{I} \alpha$. Now Lemma C.13 yields an element $x \in W$ with $x \alpha=\alpha$ such that $\operatorname{sbm}\left(w_{2}, \lambda\right) / \operatorname{sbm}\left(w_{2} s_{\alpha}, \lambda\right)$ is a homomorphic image of $Z_{\chi}(x \cdot \lambda, \alpha)$. It follows that $L_{\alpha, 2}$ is a composition factor of $Z_{\chi}(x \cdot \lambda, \alpha)$. This implies the lemma because $Z_{\chi}(x \bullet \lambda, \alpha)$ is simple by Lemma D.3.a.

D.13. We want to have an analogue to Lemma D.12 for the simple modules whose $\kappa$ invariant is a long simple root. Suppose that $R$ is of type $B_{n}, C_{n}$, or $F_{4}$; if $R$ is of type $F_{4}$ assume that $p>h+1$. Set $\alpha=\alpha_{1}$ if $R$ is of type $B_{n}$ or $F_{4}$, set $\alpha=\alpha_{n}$ if $R$ is of type $C_{n}$. So $\alpha$ is a long simple root.

Let $\chi$ be subregular with $\chi\left(\mathfrak{p}_{\alpha}\right)=0$. We claim under these assumptions:

Lemma. Let $\lambda \in C_{0}^{\prime}$. Let $\gamma$ be a long simple root with $\gamma \in J(\lambda)$. If $L$ is a simple module in $\mathcal{C}_{\lambda}$ with $\kappa(L)=\{\gamma\}$, then there exists an element $x \in W$ with $x \gamma=\alpha$ and $L \simeq Z_{\chi}(x \cdot \lambda, \alpha)$.

Proof. As in D.12 it suffices to prove the claim for one special subregular $\chi$ with $\chi\left(\mathfrak{p}_{\alpha}\right)=0$.

Consider first $R$ of type $B_{n}$. Then we may assume that $\chi$ has the standard Levi form considered in [J2], Section 3. Now the claim follows from [J2], 3.13.

Consider next $R$ of type $C_{n}$. We may assume that $\chi\left(x_{-\alpha_{i}}\right) \neq 0$ for all $i<n$. So the set $I$ as in D.7 is the set of all $\alpha_{i}$ with $i<n$, hence $W_{I} \simeq \Sigma_{n}$ the symmetric group permuting all $\varepsilon_{j}$.

Write $s_{i}=s_{\alpha_{i}}$. Set

$$
x_{i}=s_{n} s_{n-1} \ldots s_{i} \quad \text { and } \quad y_{i}=x_{1} x_{2} \ldots x_{i}
$$

for $1 \leq i \leq n$. One checks inductively that

$$
x_{i}\left(\varepsilon_{j}\right)=\left\{\begin{array}{cc}
\varepsilon_{j}, & \text { if } j<i \\
-\varepsilon_{n}, & \text { if } j=i \\
\varepsilon_{j-1}, & \text { if } j>i
\end{array}\right.
$$

and

$$
y_{i}\left(\varepsilon_{j}\right)=\left\{\begin{array}{cc}
-\varepsilon_{n+1-j}, & \text { if } j \leq i \\
\varepsilon_{j-i}, & \text { if } j>i .
\end{array}\right.
$$

We have in particular $y_{n}\left(\varepsilon_{j}\right)=-\varepsilon_{n+1-j}$ for all $j$, hence $y_{n} \in W_{I} w_{0}$. This implies

$$
\operatorname{sbm}\left(y_{n}, \lambda\right)=\operatorname{sbm}\left(w_{0}, \lambda\right)=\operatorname{soc} Z_{\chi}(\lambda) .
$$


We have (exercise) $l\left(y_{n}\right)=n(n+1) / 2$; therefore

$$
y_{n}=\left(s_{n} s_{n-1} \ldots s_{1}\right)\left(s_{n} s_{n-1} \ldots s_{2}\right) \ldots\left(s_{n} s_{n-1}\right) s_{n}
$$

is a reduced decomposition of $y_{n}$. Let $z_{k}$ be the product of the first $k$ factors on the right hand side in (2); then the $\operatorname{sbm}\left(z_{k}, \lambda\right)$ are a descending chain of submodules in $Z_{\chi}(\lambda)$. Since $L$ is not isomorphic to the simple socle of $Z_{\chi}(\lambda)$ it has to be a composition factor of $\operatorname{some} \operatorname{sbm}\left(z_{k}, \lambda\right) / \operatorname{sbm}\left(z_{k+1}, \lambda\right)$. There exists a simple root $\gamma$ with $z_{k+1}=z_{k} s_{\gamma}$; then each composition factor of $\operatorname{some} \operatorname{sbm}\left(z_{k}, \lambda\right) / \operatorname{sbm}\left(z_{k+1}, \lambda\right)$ has $\kappa$ invariant contained in $\{\gamma\}$; see D.5.a. This implies $\gamma=\alpha_{n}$.

Now a look at (2) shows that either $z_{k}=z_{0}=1$ or that $z_{k}=y_{i}$ for some $i<n$. In the first case we get that $L$ is a composition factor of

$$
Z_{\chi}(\lambda) / \operatorname{sbm}\left(s_{n}, \lambda\right) \simeq Z_{\chi}\left(\lambda, \alpha_{n}\right),
$$

hence that $L$ is isomorphic to $Z_{\chi}\left(\lambda, \alpha_{n}\right)$ since that module is simple (see D.3.a).

Suppose that we are in the second case. We have $y_{i}\left(\alpha_{n}\right)=2 \varepsilon_{n-i}$ for each $i<n$. Now

$$
2 \varepsilon_{n-i}=s_{\varepsilon_{n-i}-\varepsilon_{n}}\left(\alpha_{n}\right)
$$

shows that

$$
y_{i}\left(\alpha_{n}\right) \in W_{I} \alpha_{n}
$$

Now Lemma C.13 implies that $\operatorname{sbm}\left(y_{i}, \lambda\right) / \operatorname{sbm}\left(y_{i} s_{n}, \lambda\right)$ is a homomorphic image of $Z_{\chi}\left(x \bullet \lambda, \alpha_{n}\right)$ with $x=s_{\varepsilon_{n-i}-\varepsilon_{n}} y_{n}$ satisfying $x \alpha_{n}=\alpha_{n}$. Now the simplicity (by D.3.a) of $Z_{\chi}\left(x \bullet \lambda, \alpha_{n}\right)$ implies that this module is isomorphic to $L$; the lemma follows.

We finally turn to the case where $R$ is of type $F_{4}$. We may assume that $\chi\left(x_{-\alpha_{i}}\right)=$ 0 for all $i>1$. In other words, our set $I$ consists now of all $\alpha_{i}$ with $i>1$.

Let $w_{I}$ be the longest element in $W_{I}$. We have

$$
\operatorname{sbm}\left(w_{I} w_{0}, \lambda\right)=\operatorname{sbm}\left(w_{0}, \lambda\right)=\operatorname{soc} Z_{\chi}(\lambda) .
$$

So $L$ is a composition factor of $Z_{\chi}(\lambda) / \operatorname{sbm}\left(w_{I} w_{0}, \lambda\right)$.

An elementary calculation shows that

$$
w_{I} w_{0}=s_{1} s_{2} s_{3} s_{4} s_{2} s_{3} s_{2} s_{1} s_{2} s_{3} s_{4} s_{2} s_{3} s_{2} s_{1} \text {. }
$$

Since there are 15 factors on the right hand side, this is a reduced decomposition of $w_{I} w_{0}$. Denote by $x_{j}$ the product of the first $i$ factors on the right hand side of (3). Then the $\operatorname{sbm}\left(x_{j}, \lambda\right)$ are a descending chain of submodules in $Z_{\chi}(\lambda)$ ending in $\operatorname{sbm}\left(w_{I} w_{0}, \lambda\right)$. So $L$ is a composition factor of $\operatorname{some} \operatorname{sbm}\left(x_{j}, \lambda\right) / \operatorname{sbm}\left(x_{j+1}, \lambda\right)$.

Now suppose that $\kappa(L)=\left\{\alpha_{2}\right\}$. Then D.5.a implies $x_{j+1}=x_{j} s_{2}$, hence $j \in$ $\{1,4,6,8,11,13\}$. I claim that $x_{j} \alpha_{2} \in W_{I} \alpha_{1}$ for these six $j$. If so, then we get from Lemma C.13 that $L$ is a composition factor of some $Z_{\chi}\left(x \bullet \lambda, \alpha_{1}\right)$ with $x \alpha_{2}=\alpha_{1}$, hence by D.3.a isomorphic to such a module. Another elementary calculation shows that

$$
\begin{gathered}
x_{1} \alpha_{2}=\alpha_{1}+\alpha_{2}=s_{2} \alpha_{1}, \\
x_{4} \alpha_{2}=\alpha_{1}+\alpha_{2}+2 \alpha_{3}=s_{3} s_{2} \alpha_{1}, \\
x_{6} \alpha_{2}=\alpha_{1}+\alpha_{2}+2 \alpha_{3}+2 \alpha_{4}=s_{4} s_{3} s_{2} \alpha_{1}, \\
x_{8} \alpha_{2}=\alpha_{1}+2 \alpha_{2}+2 \alpha_{3}=s_{2} s_{3} s_{2} \alpha_{1},
\end{gathered}
$$




$$
\begin{gathered}
x_{11} \alpha_{2}=\alpha_{1}+2 \alpha_{2}+2 \alpha_{3}+2 \alpha_{4}=s_{2} s_{4} s_{3} s_{2} \alpha_{1}, \\
x_{13} \alpha_{2}=\alpha_{1}+2 \alpha_{2}+4 \alpha_{3}+2 \alpha_{4}=s_{3} s_{2} s_{4} s_{3} s_{2} \alpha_{1} .
\end{gathered}
$$

The claim follows and we are done for $i=2$. (Actually, for $j=1,4,6$ one could have quoted C.14(3) since the corresponding $x_{j}$ have the form $s_{1} w_{\beta}$ with $\beta \in W_{I} \alpha_{1}$.)

Assume now that $i=1$. The approach above does not work because $x_{7} \alpha_{1}=$ $\varpi_{1} \notin W_{I} \alpha_{1}$. Set $J=\left\{\alpha_{1}, \alpha_{2}, \alpha_{3}\right\}$ and use the abbreviation

$$
Z_{\chi, J}\left(\mu, \alpha_{1}\right)=U_{\chi}\left(\mathfrak{p}_{J}\right) \otimes_{U_{\chi}\left(\mathfrak{p}_{\alpha_{1}}\right)} L_{\chi, \alpha_{1}}(\mu)
$$

for all $\mu \in X$. Recall the notation $Z_{\chi, J}(\mu)$ from B.6(1).

The nilradical of $\mathfrak{p}_{J}$ acts trivially on each $Z_{\chi, J}(\mu)$, the centre of the standard Levi factor $\mathfrak{g}_{J}$ acts via scalars. Now $\mathfrak{g}_{J}$ is the direct sum of its centre and its derived Lie algebra $\mathcal{D} \mathfrak{g}_{J}$ [since $p \neq 2$.] So a composition series of $Z_{\chi, J}(\mu)$ is the same as that of a $\mathcal{D} \mathfrak{g}_{J}$-module.

The Lie algebra $\mathcal{D} \mathfrak{g}_{J}$ has type $B_{3}$. Assume for the moment that the restriction of $\chi$ to $\mathcal{D} \mathfrak{g}_{J}$ has the standard Levi form considered in [J2]. The results in [J2] show then that $Z_{\chi, I}(\lambda)$ has a composition series with factors (among others)

$$
Z_{\chi, J}\left(\lambda, \alpha_{1}\right) \quad \text { and } \quad Z_{\chi, J}\left(s_{\alpha_{1}+2 \alpha_{2}+2 \alpha_{3}} \bullet \lambda, \alpha_{1}\right) \text {. }
$$

[If we take $\lambda_{1}=\lambda$ in [J2], then we can take $\lambda_{5}=s_{\alpha_{1}+2 \alpha_{2}+2 \alpha_{3}} \bullet \lambda$.] Induction to $\mathfrak{g}$ yields a chain of submodules in $Z_{\chi}(\lambda)$ with

$$
Z_{\chi}\left(\lambda, \alpha_{1}\right) \quad \text { and } \quad Z_{\chi}\left(s_{\alpha_{1}+2 \alpha_{2}+2 \alpha_{3}} \cdot \lambda, \alpha_{1}\right)
$$

among the factors. Since these two modules are simple with $\kappa$ invariant $\left\{\alpha_{1}\right\}$ and since each composition series of $Z_{\chi}(\lambda)$ has only two factors with this $\kappa$ invariant, our $L$ has to be isomorphic to one of them.

It remains to be shown that we can choose $\chi$ such that its restriction of $\chi$ to $\mathcal{D} \mathfrak{g}_{J}$ has the desired standard Levi form. Well, consider $\chi$ with $\chi\left(\mathfrak{p}_{\alpha}\right)=0$ and $\chi\left(x_{-\beta}\right) \neq 0$ if and only if $\beta \in\left\{\alpha_{2}, \alpha_{3}, \alpha_{4}, \alpha_{1}+\alpha_{2}+\alpha_{3}+\alpha_{4}\right\}$. It is not difficult to check that the centraliser of $\chi$ in $\mathfrak{g}$ has dimension 6 . This implies that $\chi$ is subregular. (See [Sl], p. 38, on the connection between centralisers in $G$ and g.) On the other hand, the restriction of $\chi$ to $\mathcal{D} \mathfrak{g}_{J}$ has clearly the required form.

\section{E.}

We return to the more general set-up from Section A. We assume that $G$ satisfies (B1) and (D1).

E.1. Let $\chi \in \mathfrak{g}^{*}$ have Jordan decomposition $\chi=\chi_{s}+\chi_{n}$. Assume that we have

$$
\chi_{s}\left(\mathfrak{n}^{-}+\mathfrak{n}^{+}\right)=0 \quad \text { and } \quad \chi_{n}\left(\mathfrak{b}^{+}\right)=0 .
$$

Then the assumption that $\chi=\chi_{s}+\chi_{n}$ is a Jordan decomposition means that

$$
\chi_{s}\left(h_{\alpha}\right)=0 \quad \text { for all } \alpha \in R \text { with } \chi_{n}\left(x_{\alpha}\right) \neq 0 .
$$

Set $R_{1}$ equal to the set of all roots $\alpha$ with $\chi_{s}\left(h_{\alpha}\right)=0$. This is a root subsystem of $R$; it satisfies $R_{1}=R \cap \mathbf{Q} R_{1}$ because we assume the characteristic to be good. The centraliser $\mathfrak{l}$ of $\chi_{s}$ is given by

$$
\mathfrak{l}=\mathfrak{h} \oplus \bigoplus_{\alpha \in R_{1}} \mathfrak{g}_{\alpha}
$$


This is a Levi subalgebra of some parabolic subalgebra of $\mathfrak{g}$. This parabolic subalgebra is not uniquely determined. We choose it as follows: We first choose a basis of the root system $R_{1}$ such that $R_{1} \cap R^{+}$is the set of positive roots with respect to this basis. Then we extend this basis of $R_{1}$ to a basis of $R$. (This is possible since $R_{1}=R \cap \mathbf{Q} R_{1}$; see [Bou], Ch. VI, $\S 1$, Prop. 24.) Set $\mathfrak{u}$ equal to the direct sum of all $\mathfrak{g}_{\beta}$ with $\beta$ positive with respect to this new basis and not in $R_{1}$. Then $\mathfrak{l} \oplus \mathfrak{u}$ is a parabolic subalgebra of $\mathfrak{g}$ with nilradical $\mathfrak{u}$.

Now Kac \& Weisfeiler (or rather Friedlander \& Parshall, [FP1], Thm. 3.2) tell us: The functor $V \mapsto V^{\mathfrak{u}}$ is an equivalence of categories from $U_{\chi}(\mathfrak{g})$-modules to $U_{\chi}(\mathfrak{l})$-modules. We have

$$
\operatorname{dim}(V)=p^{\operatorname{dim} \mathfrak{u}} \operatorname{dim}\left(V^{\mathfrak{u}}\right)
$$

for all these $V$. (It is here that we need (B1) and (D1). In [FP1] one assumes also that $G$ is semi-simple, but that is not necessary; cf. [J3], 7.4.)

E.2. Keep the assumptions from E.1. We want to evaluate the functor $V \mapsto V^{\mathfrak{u}}$ on certain induced modules.

Let $I$ be a subset of the set of simple roots and let $\mathfrak{p}_{I} \supset \mathfrak{b}^{+}$be the corresponding standard parabolic subalgebra. Assume that $\chi$ satisfies in addition

$$
\chi_{s}\left(h_{\alpha}\right)=0 \quad \text { for all } \alpha \in I \quad \text { and } \quad \chi_{n}\left(\mathfrak{p}_{I}\right)=0 .
$$

Let $\mathfrak{g}_{I}$ be the standard Levi factor of $\mathfrak{p}_{I}$ (i.e., the direct sum of $\mathfrak{h}$ and all $\mathfrak{g}_{\alpha}$ with $\alpha \in R \cap \mathbf{Z} I$ ).

Let $M$ be a finite dimensional $U_{\chi}\left(\mathfrak{g}_{I}\right)$-module. Extend $M$ to a $\mathfrak{p}_{I}$-module such that the nilradical of $\mathfrak{p}_{I}$ acts trivially. We get thus a $U_{\chi}\left(\mathfrak{p}_{I}\right)$-module because $\chi$ vanishes on that nilradical. This leads then to the induced module $U_{\chi}(\mathfrak{g}) \otimes_{U_{\chi}\left(\mathfrak{p}_{I}\right)} M$. We want to describe $\left(U_{\chi}(\mathfrak{g}) \otimes_{U_{\chi}\left(\mathfrak{p}_{I}\right)} M\right)^{\mathfrak{u}}$. We shall identify each $m \in M$ with the element $1 \otimes m$ in the induced module.

We shall need some additional notation: Set $R_{2}^{+}$(resp. $R_{3}^{+}$) equal to the set of all positive roots $\beta$ with $\mathfrak{g}_{-\beta} \subset \mathfrak{u}$ (resp. $\left.\mathfrak{g}_{\beta} \subset \mathfrak{u}\right)$. So $\mathfrak{u}$ is the direct sum of all $\mathfrak{g}_{\beta}$ with $\beta \in\left(-R_{2}^{+}\right) \cup R_{3}^{+}$.

Lemma. If $m$ runs through a basis of $M$, then the set of all

$$
\prod_{\gamma>0, \gamma \in R_{1} \backslash \mathbf{Z} I} x_{-\gamma}^{r(\gamma)} \prod_{\beta \in R_{2}^{+}} x_{-\beta}^{p-1} m
$$

with $0 \leq r(\gamma)<p$ for all $\gamma \in R_{1} \backslash \mathbf{Z} I$ is a basis of $\left(U_{\chi}(\mathfrak{g}) \otimes_{U_{\chi}\left(\mathfrak{p}_{I}\right)} M\right)^{\mathfrak{u}}$.

Proof. Let me abbreviate $V=U_{\chi}(\mathfrak{g}) \otimes_{U_{\chi}\left(\mathfrak{p}_{I}\right)} M$. All

$$
\prod_{\gamma>0, \gamma \in R_{1} \backslash \mathbf{Z} I} x_{-\gamma}^{r(\gamma)} \prod_{\beta \in R_{2}^{+}} x_{-\beta}^{r(\beta)} \prod_{\beta \in R_{3}^{+}} x_{-\beta}^{r(\beta)} m
$$

with $m$ as above and all exponents running from 0 to $p-1$ are a basis of $V$. The elements in (2) are a subset of this basis, hence linearly independent.

We have $\operatorname{dim} \mathfrak{u}=\left|R_{2}^{+}\right|+\left|R_{3}^{+}\right|$. Therefore a comparison of (3) and (2) shows that the elements in (2) span a subspace of dimension equal to $\operatorname{dim}(V) / p^{\operatorname{dim} u}$. This is by E.1(4) also the dimension of $V^{\mathfrak{u}}$. So the claim will follow if we can show that all elements in (2) belong to $V^{\mathfrak{u}}$. 
There exists a group homomorphism $d: \mathbf{Z} R \rightarrow \mathbf{Z}$ with $d(\alpha)=0$ for all $\alpha \in R_{1}$ and $d(\beta)>0$ for all $\beta \in R$ with $\mathfrak{g}_{\beta} \subset \mathfrak{u}$, i.e., for all $\beta \in\left(-R_{2}^{+}\right) \cup R_{3}^{+}$. (If $\alpha$ belongs to the basis of $R$ used to construct $\mathfrak{u}$ in E.1, then set $d(\alpha)=1$ if $\alpha \notin R_{1}$ and $d(\alpha)=0$ otherwise.) We get then a $\mathbf{Z}$-grading on $\mathfrak{g}$ such that each $\mathfrak{g}_{\beta}$ is homogeneous of degree $d(\beta)$ and such that $\mathfrak{h}$ is homogeneous of degree 0 . Then $\mathfrak{l}$ is the homogeneous component of degree 0 and $\mathfrak{u}$ is the sum of the homogeneous components of positive degree. This induces a grading on $U(\mathfrak{g})$ and then also on $U_{\chi}(\mathfrak{g})$. (For the last claim one uses that $\chi$ vanishes by E.1(2) on all $x_{\beta}$ not in l.)

The subalgebra $\mathfrak{p}_{I}$ of $\mathfrak{g}$ is graded (since it is spanned by $\mathfrak{h}$ and certain $x_{\beta}$, hence by homogeneous elements). If we give $M$ the trivial grading (where everything has degree 0 ), then it becomes a graded $\mathfrak{p}_{I}$-module. (The homogeneous components of $\mathfrak{p}_{I}$ of non-zero degree belong to the nilradical of $\mathfrak{p}_{I}$ and act as 0 .) This leads then to a grading on the induced $U_{\chi}(\mathfrak{g})$-module $V$.

Each basis element in (3) is homogeneous [of degree $\left.-\sum r(\beta) d(\beta)\right]$. We get elements of maximal degree if we choose $r(\beta)=p-1$ whenever $d(\beta)<0$, and $r(\beta)=0$ whenever $d(\beta)>0$. We have to admit arbitrary $m$ and arbitrary $r(\beta)$ whenever $d(\beta)=0$. If $\beta \in R^{+}$, then we have $d(\beta)=0$ if and only if $\beta \in R_{1}$, and $d(\beta)<0$ if and only if $\mathfrak{g}_{-\beta} \subset \mathfrak{u}$ if and only if $\beta \in R_{2}^{+}$. It follows that the elements in (2) are precisely the elements in (3) that have maximal degree. So they are a basis for the homogeneous component of maximal degree of $V$. Since each $x_{\beta} \in \mathfrak{u}$ maps an element of some degree to an element of higher degree, all elements in (2) are annihilated by $\mathfrak{u}$. The lemma follows.

E.3. Lemma. We have

$$
x_{\gamma} \prod_{\beta \in R_{2}^{+}} x_{-\beta}^{p-1} m=\prod_{\beta \in R_{2}^{+}} x_{-\beta}^{p-1} x_{\gamma} m
$$

for all $\gamma \in R_{1}$ and $m \in M$.

Proof. Let me abbreviate $x=\prod_{\beta \in R_{2}^{+}} x_{-\beta}^{p-1}$. We have to show that $x_{\gamma} x-x x_{\gamma}$ annihilates $M \subset V$.

Since $\mathfrak{u}$ is the nilradical of $\mathfrak{l} \oplus \mathfrak{u}$, we have $[\mathfrak{l}, \mathfrak{u}] \subset \mathfrak{u}$. So $x_{\gamma} \in \mathfrak{l}$ implies that

$$
x_{\gamma} x-x x_{\gamma} \in U(\mathfrak{u}) .
$$

We can express this commutator in terms of a PBW basis of $U(\mathfrak{u})$. So $x_{\gamma} x-x x_{\gamma}$ is a linear combination of monomials of the form

$$
\prod_{\beta \in R_{2}^{+}} x_{-\beta}^{a(\beta)} \prod_{\beta \in R_{3}^{+}} x_{\beta}^{b(\beta)}
$$

with non-negative integers $a(\beta), b(\beta)$.

Now $x_{\gamma} x-x x_{\gamma}$ and all monomials in (3) are eigenvectors for the adjoint action of $T$. We can therefore assume that only monomials occur that have the same weight as $x_{\gamma} x-x x_{\gamma}$, i.e., with

$$
\sum_{\beta \in R_{3}^{+}} b(\beta) \beta-\sum_{\beta \in R_{2}^{+}} a(\beta) \beta=\gamma-(p-1) \sum_{\beta \in R_{2}^{+}} \beta .
$$

If $b(\beta)>0$ for some $\beta \in R_{3}^{+}$, then the term in (3) annihilates $M$ since $x_{\beta}$ belongs to the nilradical of $\mathfrak{p}_{I}$, hence annihilates $M$. In order to prove our claim it therefore 
suffices to look at the terms in (3) with $b(\beta)=0$ for all $\beta \in R_{3}^{+}$. Then (4) reduces to

$$
\sum_{\beta \in R_{2}^{+}}(p-1-a(\beta)) \beta=\gamma .
$$

If $a(\beta) \geq p$ for some $\beta \in R_{2}^{+}$, then $x_{-\beta}^{a(\beta)}$ acts as 0 on $V$ since $\chi(\mathfrak{u})=0$; hence so does the monomial in (3). So we may assume that $a(\beta) \leq p-1$ for all $\beta \in R_{2}^{+}$. Note that then $a(\beta)<p-1$ for at least one $\beta$, since otherwise we get $0=\gamma$.

Recall now the homomorphism $d: \mathbf{Z} R \rightarrow \mathbf{Z}$ used in the proof of Lemma E.2. It satisfies $d(\gamma)=0$ since $\gamma \in R_{1}$, and $d(\beta)<0$ for all $\beta \in R_{2}^{+}$. If we assume in (5) that $a(\beta) \leq p-1$ for all $\beta$ and $a(\beta)<p-1$ for at least one $\beta$, then the left hand side has a negative image under $d$. This contradicts $d(\gamma)=0$. So (5) cannot have a solution with $a(\beta) \leq p-1$ for all $\beta \in R_{2}^{+}$. Therefore all monomials from (3) annihilate $M$; the claim follows.

E.4. Set $\rho^{\prime}$ equal to half the sum of the positive roots in $R_{1}$.

Lemma. We have $\left\langle\rho-\rho^{\prime}, \gamma^{\vee}\right\rangle=\sum_{\beta \in R_{2}^{+}}\left\langle\beta, \gamma^{\vee}\right\rangle$ for all $\gamma \in R_{1}$.

Proof. Let $\gamma \in R_{1}$. Set $a_{i}=\sum_{\beta \in R_{i}^{+}}\left\langle\beta, \gamma^{\vee}\right\rangle$ for $i=2,3$. Since $R^{+}$is the disjoint union of $R^{+} \cap R_{1}, R_{2}^{+}$, and $R_{3}^{+}$, we get

$$
\left\langle\rho-\rho^{\prime}, \gamma^{\vee}\right\rangle=\frac{1}{2}\left(a_{2}+a_{3}\right) .
$$

Because $\mathfrak{u}$ is the nilradical of a parabolic subalgebra with Levi factor $\mathfrak{l} \supset \mathfrak{h}$, the set of all $\beta$ with $\mathfrak{g}_{\beta} \subset \mathfrak{u}$ is stable under the Weyl group of $\mathfrak{l}$. Now $s_{\gamma}$ belongs to that Weyl group; this implies $s_{\gamma}\left(\left(-R_{2}^{+}\right) \cup R_{3}^{+}\right)=\left(-R_{2}^{+}\right) \cup R_{3}^{+}$, hence

$$
0=\sum_{\beta \in\left(-R_{2}^{+}\right) \cup R_{3}^{+}}\left\langle\beta, \gamma^{\vee}\right\rangle=-a_{2}+a_{3} .
$$

So $a_{2}=a_{3}$; plugging this into (1) we get our claim.

E.5. Set

$$
\delta_{1}=\sum_{\beta \in R_{2}^{+}} \beta
$$

Each $\alpha \in I$ belongs to the basis of $R$ with positive system $R^{+}$, and it belongs to the basis of $R_{1}$ with positive system $R^{+} \cap R_{1}$. This implies $\left\langle\rho, \alpha^{\vee}\right\rangle=1=\left\langle\rho^{\prime}, \alpha^{\vee}\right\rangle$. So Lemma E. 4 shows that $\left\langle\delta_{1}, \alpha^{\vee}\right\rangle=0$, hence $\delta_{1}\left(h_{\alpha}\right)=0$ for all $\alpha \in I$. It follows that $\delta_{1}$ vanishes on the intersection of $\mathfrak{h}$ with the derived Lie algebra of $\mathfrak{g}_{I}$. We get therefore a one dimensional $\mathfrak{g}_{I}$-module where each $x_{\beta}$ with $\beta \in R \cap \mathbf{Z} I$ acts as 0 , and each $h \in \mathfrak{h}$ as $\delta_{1}(h)$. This is a restricted $\mathfrak{g}_{I}$-module. Its tensor product with a $U_{\chi}\left(\mathfrak{g}_{I}\right)$-module, say $N$, is again a $U_{\chi}\left(\mathfrak{g}_{I}\right)$-module; we shall denote it by $N \otimes \delta_{1}$.

Proposition. Let $M$ be a finite dimensional $U_{\chi}\left(\mathfrak{g}_{I}\right)$-module extended trivially to a $U_{\chi}\left(\mathfrak{p}_{I}\right)$-module. Then we have an isomorphism of $U_{\chi}(\mathfrak{l})$-modules

$$
\left(U_{\chi}(\mathfrak{g}) \otimes_{U_{\chi}\left(\mathfrak{p}_{I}\right)} M\right)^{\mathfrak{u}} \simeq U_{\chi}(\mathfrak{l}) \otimes_{U_{\chi}\left(\mathfrak{l} \cap \mathfrak{p}_{I}\right)}\left(M \otimes \delta_{1}\right) .
$$

Proof. Abbreviate the left hand side in (2) by $M^{\prime}$. Write $x=\prod_{\beta \in R_{2}^{+}} x_{-\beta}^{p-1}$. Lemma E.3 implies that the subspace $x M$ of $M^{\prime}$ is stable under all $x_{\gamma}$ satisfying $\gamma \in R_{1} \cap R^{+}$ 
or $\gamma \in\left(-R^{+}\right) \cap \mathbf{Z} I$. (Note that $M$ is stable under these $x_{\gamma}$.) On the other hand, we have for all $h \in \mathfrak{h}$ and $m \in M$

$$
h x m=x h m-\sum_{\beta \in R_{2}^{+}}(p-1) \beta(h) x m=x h m+\delta_{1}(h) x m .
$$

Because $\mathfrak{l} \cap \mathfrak{p}_{I}$ is spanned by $\mathfrak{h}$ and the $x_{\gamma}$ as above, it follows that $x M$ is a $U_{\chi}\left(\mathfrak{l} \cap \mathfrak{p}_{I}\right)$-submodule of $M^{\prime}$. Formula (3) and Lemma E.3 show that this submodule is isomorphic to $M \otimes \delta_{1}$.

The universal property of an induced module yields a homomorphism of $U_{\chi}(\mathfrak{l})-$ modules

$$
U_{\chi}(\mathfrak{l}) \otimes_{U_{\chi}\left(\mathfrak{I} \cap p_{I}\right)} x M \rightarrow M^{\prime}, \quad u \otimes x m \mapsto u x m .
$$

Lemma E.2 implies that this map is bijective. The claim follows.

E.6. We now drop the assumptions fixed throughout E.1-E.5 and consider a more general situation.

Let $\chi=\chi_{0}+\chi_{1} \in \mathfrak{g}^{*}$ with

$$
\chi_{0}\left(\mathfrak{n}^{-}+\mathfrak{n}^{+}\right)=0 \quad \text { and } \quad \chi_{1}\left(\mathfrak{b}^{+}\right)=0 .
$$

So this looks like E.1(1); but we no longer assume that $\chi=\chi_{0}+\chi_{1}$ is a Jordan decomposition, i.e., the analogue of E.1(2) will not hold in general.

On the other hand, we fix again a set $I$ as in E.2 and assume the analogue of E.2(1):

$$
\chi_{0}\left(h_{\alpha}\right)=0 \quad \text { for all } \alpha \in I \quad \text { and } \quad \chi_{1}\left(\mathfrak{p}_{I}\right)=0 .
$$

Let $P_{I}$ be standard parabolic subgroup of $G$ with Lie algebra $\mathfrak{p}_{I}$. Denote its unipotent radical by $R_{u}\left(P_{I}\right)$. For each $g \in R_{u}\left(P_{I}\right)$ and all $x \in \mathfrak{p}_{I}$ the difference $\operatorname{Ad}\left(g^{-1}\right)(x)-x$ belongs to the nilradical of $\mathfrak{p}_{I}$. Since $\chi$ vanishes on that nilradical, we get

$$
(g \cdot \chi)_{\mid \mathfrak{p}_{I}}=\chi_{\mathfrak{p}_{I}} .
$$

$\left(\right.$ Recall that $(g \cdot \chi)(x)=\chi\left(\operatorname{Ad}\left(g^{-1}\right)(x)\right)$.)

Lemma. There exist $g \in R_{u}\left(P_{I}\right)$ and $\chi_{1}^{\prime} \in \mathfrak{g}^{*}$ with $\chi_{1}^{\prime}\left(\mathfrak{p}_{I}\right)=0$ such that $g \cdot \chi$ has Jordan decomposition $g \cdot \chi=\chi_{0}+\chi_{1}^{\prime}$.

Proof. This follows from the proof in Subsection 3.8 in $[\mathrm{KW}]$. If we apply the construction there to our $\chi$ (as their $l$ ), then we get an element $g \in G$ such that $g \cdot \chi$ has Jordan decomposition $g \cdot \chi=\chi_{0}+\chi_{1}^{\prime}$ where $\chi_{1}^{\prime}=g \cdot \chi-\chi_{0}$. We just have to check that the $g$ used there actually is in $R_{u}\left(P_{I}\right)$. (If so, then we get $\chi_{1}^{\prime}\left(\mathfrak{p}_{I}\right)=0$ from $\chi_{1}\left(\mathfrak{p}_{I}\right)=0$, since $\chi_{1}^{\prime}$ coincides by (3) with $\chi_{1}$ on $\mathfrak{p}_{I}$.)

Well, in $[\mathrm{KW}]$ one considers the set (denoted by $\Pi$ ) of all positive roots $\beta$ with $\chi\left(h_{\beta}\right) \neq 0$. Then $g$ is constructed as a product of elements from the root subgroups $U_{\beta}$ with $\beta \in \Pi$. Now our assumption (2) says that $\Pi \subset R^{+} \backslash \mathbf{Z} I$. This implies that $g \in R_{u}\left(P_{I}\right)$ as claimed.

E.7. Keep the assumptions of E.6 and choose $g$ as in Lemma E.6. We can apply E.1-E.5 with $\chi_{s}=\chi_{0}, \chi_{n}=\chi_{1}^{\prime}$ and with $\chi$ replaced by $g \chi$. Set in particular $R_{1}=\left\{\alpha \in R \mid \chi_{0}\left(h_{\alpha}\right)=0\right\}$ and $\mathfrak{l}=\mathfrak{h} \oplus \bigoplus_{\alpha \in R_{1}} \mathfrak{g}_{\alpha}$. We get also $\delta_{1}$ as in E.5(1). 
Proposition. There exists an equivalence of categories

$$
\mathcal{F}: U_{\chi}(\mathfrak{g}) \text {-modules } \rightarrow U_{g \chi}(\mathfrak{l}) \text {-modules }
$$

such that

$$
\mathcal{F}\left(U_{\chi}(\mathfrak{g}) \otimes_{U_{\chi}\left(\mathfrak{p}_{I}\right)} M\right) \simeq U_{g \chi}(\mathfrak{l}) \otimes_{U_{\chi}\left(\mathfrak{I} \cap \mathfrak{p}_{I}\right)}\left(M \otimes \delta_{1}\right)
$$

for all finite dimensional $U_{\chi}\left(\mathfrak{g}_{I}\right)$-modules $M$ extended trivially to a $\mathfrak{p}_{I}$-module.

Proof. We construct $\mathcal{F}$ as a composition of two equivalences. The first one is

$$
U_{\chi}(\mathfrak{g}) \text {-modules } \rightarrow U_{g \chi}(\mathfrak{g}) \text {-modules, } \quad V \mapsto{ }^{g} V
$$

where ${ }^{g} V$ is equal to $V$ as a vector space and where each $x \in \mathfrak{g}$ acts on ${ }^{g} V$ as $\operatorname{Ad}\left(g^{-1}\right)(x)$ acts on $V$.

In case $V=U_{\chi}(\mathfrak{g}) \otimes_{U_{\chi}\left(\mathfrak{p}_{I}\right)} M$ with $M$ as above one checks easily that

$$
{ }^{g} V \simeq U_{g \chi}(\mathfrak{g}) \otimes_{U_{g \chi}\left(\operatorname{Ad}(g) \mathfrak{p}_{I}\right)}{ }^{g} M \simeq U_{g \chi}(\mathfrak{g}) \otimes_{U_{\chi}\left(\mathfrak{p}_{I}\right)}{ }^{g} M .
$$

For the second equality use that $\operatorname{Ad}(g) \mathfrak{p}_{I}=\mathfrak{p}_{I}$ since $g \in R_{u}\left(P_{I}\right)$ and apply E.6(3). For each $x \in \mathfrak{p}_{I}$ the difference $\operatorname{Ad}\left(g^{-1}\right) x-x$ belongs to the nilradical of $\mathfrak{p}_{I}$. This nilradical acts trivially on $M$. Therefore ${ }^{g} M$ is isomorphic to $M$ as a $\mathfrak{p}_{I}$-module. We get thus

$$
{ }^{g} V \simeq U_{g \chi}(\mathfrak{g}) \otimes_{U_{\chi}\left(\mathfrak{p}_{I}\right)} M
$$

Let $\mathcal{F}$ be the composition of the functor in (2) with the equivalence of categories $V^{\prime} \mapsto\left(V^{\prime}\right)^{\mathfrak{u}}$ from [FP1], with $\mathfrak{u}$ as in E.1. Then $\mathcal{F}$ is an equivalence of categories from $U_{\chi}(\mathfrak{g})$-modules to $U_{g \chi}(\mathfrak{l})$-modules with $\mathcal{F}(V)=\left({ }^{g} V\right)^{\mathfrak{u}}$. Combining (3) and Proposition E.5 we get (1).

F.

We assume in this section that (B1), (B2), and (D1) hold. From F.5 on we shall also assume (D2).

F.1. Let $\alpha$ be a simple root. We write (as before) $\mathfrak{p}_{\alpha}=\mathfrak{b}^{+} \oplus \mathfrak{g}_{-\alpha}$ for the corresponding minimal parabolic subalgebra of $\mathfrak{g}$. Let $\gamma$ be a root orthogonal to $\alpha$.

We want to show:

Proposition. Suppose that the root system $R \cap(\mathbf{Q} \alpha+\mathbf{Q} \gamma)$ has type $A_{1} \times A_{1}$ or $B_{2}$. In the second case assume that $\alpha$ is a short root in that subsystem. Then we have

$$
\operatorname{Hom}_{\mathfrak{g}}\left(Z_{\chi}\left(s_{\gamma} \bullet \mu, \alpha\right), Z_{\chi}(\mu, \alpha)\right) \neq 0
$$

for all $\mu \in X$ and all $\chi \in \mathfrak{g}^{*}$ with $\chi\left(\mathfrak{p}_{\alpha}\right)=0$.

The proof will occupy the following subsections until F.4. We shall first prove the proposition in the rank 2 case (i.e., if $R=R \cap(\mathbf{Q} \alpha+\mathbf{Q} \gamma)$ ) and then use results from Sections $E$ and A to reduce to that case.

Remark. This proposition does not generalise to the situation where $R \cap(\mathbf{Q} \alpha+\mathbf{Q} \gamma)$ has type $B_{2}$ and where $\alpha$ is long. For example, if $R$ is of type $B_{2}$, then [J2], 3.13 shows that there exist simple modules $Z_{\chi}\left(\lambda_{1}, \alpha_{1}\right)$ and $Z_{\chi}\left(\lambda_{3}, \alpha_{1}\right)$ that are not isomorphic to each other, but where $\lambda_{3}=s_{\gamma} \bullet \lambda_{1}$ with $\gamma$ the positive root orthogonal to $\alpha_{1}$. 
F.2. Lemma. Proposition F.1 holds if $R$ is of type $A_{1} \times A_{1}$.

Proof. In this case also $\gamma$ is a simple root. Let $e$ be the integer with $0 \leq e<p$ and $\left\langle\mu+\rho, \alpha^{\vee}\right\rangle \equiv e(\bmod p)$. Let $v\left(\right.$ resp. $\left.v^{\prime}\right)$ be the standard generator of $Z_{\chi}(\mu, \alpha)$ (resp. of $\left.Z_{\chi}\left(s_{\gamma} \bullet \mu, \alpha\right)\right)$. Using $\left[x_{ \pm \alpha}, x_{-\gamma}\right]=0$, one checks easily that there exists a homomorphism between our two modules that maps $v^{\prime}$ to $x_{-\gamma}^{e} v$.

F.3. Lemma. Proposition F.1 holds if $R$ is of type $C_{2}$.

Proof. The assumption (D1) implies in this case that $p \neq 2$. It follows that the derived Lie algebra of $\mathfrak{g}$ is simple and that $\mathfrak{g}$ is the direct sum of its centre and of its derived Lie algebra $\mathcal{D} \mathfrak{g}$. The centre (equal to the intersection in $\mathfrak{h}$ of all $\operatorname{ker}(\beta)$ with $\beta \in R)$ acts on $Z_{\chi}(\mu, \alpha)$ and $Z_{\chi}\left(s_{\gamma} \bullet \mu, \alpha\right)$ via the same linear form. So we can replace $\mathfrak{g}$ in F.1(1) by $\mathcal{D} \mathfrak{g}$. When we regard a $Z_{\chi}(\nu)$ as a $\mathcal{D} \mathfrak{g}$-module we get again a baby Verma module. An analogous result holds for the $Z_{\chi}(\nu, \alpha)$. Therefore it suffices to prove the claim for $\mathcal{D} \mathfrak{g}$ instead of $\mathfrak{g}$.

So assume from now on that $G$ is semi-simple. Let $\beta$ be the second simple root (besides $\alpha$ ). Recall that we suppose in this case that $\alpha$ is short, hence $\beta$ long. Since $\gamma$ is orthogonal to $\alpha$, we have $\gamma \in\{ \pm(\alpha+\beta)\}$ and $s_{\gamma}=s_{\beta} s_{\alpha} s_{\beta}$.

Assume that $\chi$ is subregular with $\chi\left(x_{-\beta}\right) \neq 0$. (Such $\chi$ are by D.1 dense in the set $\Xi$ of all $\chi \in \mathfrak{g}^{*}$ with $\chi\left(\mathfrak{p}_{\alpha}\right)=0$. Therefore Proposition A.7 implies that it suffices to prove the claim for these $\chi$.)

Since $C_{0}$ is a fundamental domain for the affine Weyl group, there exist $\lambda \in C_{0}$, $w \in W$, and $\nu \in X$ with $\mu=w \cdot \lambda+p \nu$. We can actually assume that $\lambda \in C_{0}^{\prime}$ : If $\lambda \in C_{0} \backslash C_{0}^{\prime}$, then $\left\langle\lambda+\rho, \gamma^{\vee}\right\rangle=p$. In this case one checks (using $p \neq 2$ ) that $\lambda^{\prime}=s_{\beta} w_{0} \bullet \lambda+p \varpi_{\alpha}$ belongs to $C_{0}^{\prime}$ and replaces $\lambda$ by $\lambda^{\prime}$.

Because any $Z_{\chi}\left(\mu_{1}, \alpha\right)$ depends only on the coset of $\mu_{1}$ modulo $p X$, we see that it suffices to show that

$$
\operatorname{Hom}_{\mathfrak{g}}\left(Z_{\chi}\left(s_{\gamma} w \bullet \lambda, \alpha\right), Z_{\chi}(w \bullet \lambda, \alpha)\right) \neq 0
$$

for all $\lambda \in C_{0}^{\prime}$ and $w \in W$.

This is now done case-by-case. We first assume that $\lambda$ is regular, i.e., that $\left\langle\lambda+\rho, \alpha^{\vee}\right\rangle>0$ and $\left\langle\lambda+\rho, \beta^{\vee}\right\rangle>0$. Then $Z_{\chi}(\lambda)$ has length 4 . We denote the composition factors as in D.6 by $L_{\alpha}$ [short for $L_{\alpha, 1}$ ], $L_{\beta, 1}, L_{\beta, 2}$, and $L_{0}$. (We know in this case actually that $L_{\beta, 1}$ and $L_{\beta, 2}$ are not isomorphic to each other; but that will not play a role here.)

The chain of submodules from D.7(3) is in our situation

$$
Z_{\chi}(\lambda) \supset M^{\alpha} \supset M^{\alpha+\beta} \supset 0 .
$$

We have

$$
\begin{gathered}
Z_{\chi}(\lambda)=\operatorname{sbm}(1, \lambda)=\operatorname{sbm}\left(s_{\beta}, \lambda\right), \\
M^{\alpha}=\operatorname{sbm}\left(s_{\alpha}, \lambda\right)=\operatorname{sbm}\left(s_{\beta} s_{\alpha}, \lambda\right),
\end{gathered}
$$

and

$$
M^{\alpha+\beta}=\operatorname{sbm}\left(s_{\alpha} s_{\beta}, \lambda\right)=\operatorname{sbm}\left(s_{\beta} s_{\alpha} s_{\beta}, \lambda\right)=\operatorname{sbm}\left(s_{\alpha} s_{\beta} s_{\alpha}, \lambda\right)=\operatorname{sbm}\left(w_{0}, \lambda\right),
$$

where the equality $M^{\alpha+\beta}=\operatorname{sbm}\left(w_{0}, \lambda\right)$ was observed in D.9, right after D.9(1).

We have $Z_{\chi}(\lambda) / M^{\alpha} \simeq L_{\alpha}$ and $M^{\alpha+\beta} \simeq L_{0}$. Let me use the abbreviation $M=$ $M^{\alpha} / M^{\alpha+\beta}$. This module has length 2 with composition factors $L_{\beta, 1}$ and $L_{\beta, 2}$. 
We have $w^{-1} \alpha=\alpha$ for $w=1$ and $w=s_{\gamma}$. Therefore Lemma D.3.a and Lemma D.5.c imply that $Z_{\chi}(w \bullet \lambda, \alpha)$ is [for these two $w$ ] simple with $\kappa$ invariant $\{\alpha\}$, hence satisfies

$$
Z_{\chi}(\lambda, \alpha) \simeq L_{\alpha} \simeq Z_{\chi}\left(s_{\gamma} \cdot \lambda, \alpha\right) .
$$

We have $w^{-1} \alpha=-\alpha_{0}$ for $w=s_{\alpha} s_{\beta}$ and $w=s_{\alpha} s_{\beta} s_{\alpha}$, hence by D.4

$$
Z_{\chi}\left(s_{\alpha} s_{\beta} \bullet \lambda, \alpha\right) \simeq L_{0} \simeq Z_{\chi}\left(s_{\alpha} s_{\beta} s_{\alpha} \cdot \lambda, \alpha\right) .
$$

Clearly (2) and (3) imply (1) for all $w \in\left\{1, s_{\alpha} s_{\beta}, s_{\alpha} s_{\beta} s_{\alpha}, s_{\beta} s_{\alpha} s_{\beta}\right\}$

In order to get the result for the remaining $w \in W$, we recall that we have for all $w \in W$ an exact sequence

$$
0 \rightarrow Z_{\chi}\left(s_{\alpha} w \bullet \lambda, \alpha\right) \longrightarrow Z_{\chi}(w \bullet \lambda) \longrightarrow Z_{\chi}(w \bullet \lambda, \alpha) \rightarrow 0 .
$$

Using (2) and (3) we see that the remaining $Z_{\chi}\left(w_{\bullet} \lambda, \alpha\right)$ have composition factors

$$
\begin{array}{ll}
L_{\beta, 1}, L_{\beta, 2}, L_{0} & \text { for } w=s_{\alpha} \text { and } w=w_{0}, \\
L_{\beta, 1}, L_{\beta, 2}, L_{\alpha} & \text { for } w=s_{\beta} \text { and } w=s_{\beta} s_{\alpha} .
\end{array}
$$

Note that $w_{0}=s_{\gamma} s_{\alpha}$ and $s_{\beta} s_{\alpha}=s_{\gamma} s_{\beta}$.

We have $M^{\alpha} \simeq Z_{\chi}\left(s_{\alpha} \bullet \lambda, \alpha\right)$ by C.12(4), hence a short exact sequence

$$
0 \rightarrow L_{0} \longrightarrow Z_{\chi}\left(s_{\alpha} \bullet \lambda, \alpha\right) \longrightarrow M \rightarrow 0 .
$$

The isomorphism $Z_{\chi}(\lambda) \stackrel{\sim}{\longrightarrow} Z_{\chi}\left(s_{\beta} \bullet \lambda\right)$ induces a surjective homomorphism from $Z_{\chi}(\lambda)$ onto $Z_{\chi}\left(s_{\beta} \bullet \lambda, \alpha\right)$. The kernel of this map has to be isomorphic to $L_{0}$, hence equal to the socle $M^{\alpha+\beta}$ of $Z_{\chi}(\lambda)$. This leads to a short exact sequence

$$
0 \rightarrow M \longrightarrow Z_{\chi}\left(s_{\beta} \bullet \lambda, \alpha\right) \longrightarrow L_{\alpha} \rightarrow 0 .
$$

The isomorphism $Z_{\chi}\left(s_{\alpha} \bullet \lambda\right) \stackrel{\sim}{\longrightarrow} Z_{\chi}\left(s_{\beta} s_{\alpha} \bullet \lambda\right)$ induces a surjective homomorphism from $Z_{\chi}\left(s_{\alpha} \bullet \lambda\right)$ onto $Z_{\chi}\left(s_{\beta} s_{\alpha} \bullet \lambda, \alpha\right)$. The kernel of this map has to be isomorphic to $L_{0}$. Therefore the submodule $Z_{\chi}(\lambda, \alpha) \simeq L_{\alpha}$ of $Z_{\chi}\left(s_{\alpha} \cdot \lambda\right)$ is mapped isomorphically onto a submodule of $Z_{\chi}\left(s_{\beta} s_{\alpha} \bullet \lambda, \alpha\right)$. The corresponding factor module of $Z_{\chi}\left(s_{\beta} s_{\alpha} \bullet \lambda, \alpha\right)$ is a homomorphic image of $Z_{\chi}\left(s_{\alpha} \bullet \lambda, \alpha\right)$. The surjection from $Z_{\chi}\left(s_{\alpha} \bullet \lambda, \alpha\right)$ onto this image has kernel isomorphic to $L_{0}$. Since $Z_{\chi}\left(s_{\alpha} \bullet \lambda, \alpha\right)$ has only one submodule isomorphic to $L_{0}$, that image has to be isomorphic to $M$. We get therefore a short exact sequence

$$
0 \rightarrow L_{\alpha} \longrightarrow Z_{\chi}\left(s_{\beta} s_{\alpha} \cdot \lambda, \alpha\right) \longrightarrow M \rightarrow 0 .
$$

Applying (4) with $s_{\alpha} w=w_{0}$, we see that $Z_{\chi}\left(w_{0} \bullet \lambda, \alpha\right)$ is isomorphic to a submodule of $Z_{\chi}\left(s_{\beta} s_{\alpha} s_{\beta} \bullet \lambda\right)$, hence to one of $Z_{\chi}\left(s_{\alpha} s_{\beta} \bullet \lambda\right)$. Here we also have a submodule isomorphic to $Z_{\chi}\left(s_{\beta} \bullet \lambda, \alpha\right)$. A look at the composition factors shows that the sum of these two submodules has to be all of $Z_{\chi}\left(s_{\alpha} s_{\beta} \cdot \lambda\right)$ and that their intersection has to have composition factors $L_{\beta, 1}$ and $L_{\beta, 2}$. Another look at the composition factors shows that $Z_{\chi}\left(s_{\beta} \bullet \lambda, \alpha\right)$ has only one submodule with this property; this submodule is by (6) isomorphic to $M$. Therefore also $Z_{\chi}\left(w_{0} \bullet \lambda, \alpha\right)$ has a submodule isomorphic to $M$; we get thus a short exact sequence

$$
0 \rightarrow M \longrightarrow Z_{\chi}\left(w_{0} \bullet \lambda, \alpha\right) \longrightarrow L_{0} \rightarrow 0
$$

Comparing (5) with (8) and (6) with (7) we get now (1) also for the remaining four elements in $W$. 
We now turn to singular $\lambda$. Suppose first that $\left\langle\lambda+\rho, \alpha^{\vee}\right\rangle>0$ and $\left\langle\lambda+\rho, \beta^{\vee}\right\rangle=0$. Then $Z_{\chi}(\lambda)$ has length 2 with composition factors $L_{\alpha}$ and $L_{0}$. We have now

$$
Z_{\chi}\left(s_{\beta} \bullet \lambda, \alpha\right)=Z_{\chi}(\lambda, \alpha) \simeq L_{\alpha} \simeq Z_{\chi}\left(s_{\gamma} \bullet \lambda, \alpha\right)=Z_{\chi}\left(s_{\beta} s_{\alpha} \bullet \lambda, \alpha\right)
$$

and

$$
Z_{\chi}\left(s_{\alpha} \bullet \lambda, \alpha\right)=Z_{\chi}\left(s_{\alpha} s_{\beta} \bullet \lambda, \alpha\right) \simeq L_{0} \simeq Z_{\chi}\left(s_{\alpha} s_{\beta} s_{\alpha} \bullet \lambda, \alpha\right)=Z_{\chi}\left(w_{0} \bullet \lambda, \alpha\right) .
$$

So the claim follows in this case.

If $\left\langle\lambda+\rho, \alpha^{\vee}\right\rangle=0=\left\langle\lambda+\rho, \beta^{\vee}\right\rangle$, then $w_{\bullet} \lambda=\lambda$ for all $w \in W$ and the claim is trivial. So we are left with the case where $\left\langle\lambda+\rho, \alpha^{\vee}\right\rangle=0$ and $\left\langle\lambda+\rho, \beta^{\vee}\right\rangle>0$. We have now $w_{\bullet} \lambda=s_{\gamma} w_{\bullet} \lambda$ for all $w \in\left\{s_{\beta}, s_{\beta} s_{\alpha}, s_{\alpha} s_{\beta}, s_{\alpha} s_{\beta} s_{\alpha}\right\}$, so the claim is trivial for these elements. On the other hand, we have now

$$
Z_{\chi}\left(s_{\alpha} \bullet \lambda, \alpha\right)=Z_{\chi}(\lambda, \alpha)=Z_{\chi}(\lambda) \simeq Z_{\chi}\left(s_{\beta} \bullet \lambda\right)
$$

and

$$
Z_{\chi}\left(w_{0} \bullet \lambda, \alpha\right)=Z_{\chi}\left(s_{\beta} s_{\alpha} s_{\beta} \bullet \lambda, \alpha\right)=Z_{\chi}\left(s_{\beta} s_{\alpha} s_{\beta} \bullet \lambda\right) \simeq Z_{\chi}\left(s_{\alpha} s_{\beta} \bullet \lambda\right) .
$$

So it suffices to show that there are non-zero homomorphisms (in both directions) between $Z_{\chi}\left(s_{\beta} \bullet \lambda\right)$ and $Z_{\chi}\left(s_{\alpha} s_{\beta} \bullet \lambda\right)$. But there exists a non-zero homomorphism from $Z_{\chi}\left(s_{\alpha} \bullet \mu\right)$ to $Z_{\chi}(\mu)$ for all $\mu \in X$ : This is trivial in case $\left\langle\mu+\rho, \alpha^{\vee}\right\rangle \in \mathbf{Z}_{p}$; otherwise take $\varphi_{\alpha}^{\mu}$ as in A.4(2).

F.4. We now begin the proof of Proposition F.1 in general. Set

$$
\mathfrak{X}_{1}=\left\{f \in \mathfrak{h}^{*} \mid f\left(h_{\alpha}\right)=\mu\left(h_{\alpha}\right), f\left(h_{\gamma}\right)=\mu\left(h_{\gamma}\right)\right\} .
$$

Recall the notation $Z(f, \chi, \alpha)$ from A.6(3). Note that $Z_{\chi}(\mu, \alpha)=Z(\mu, \chi, \alpha)$ and $Z_{\chi}\left(s_{\gamma} \bullet \mu, \alpha\right)=Z(\mu-a \gamma, \chi, \alpha)$ where $a$ is the integer with $0 \leq a<p$ and $a \equiv$ $\left\langle\mu+\rho, \gamma^{\vee}\right\rangle$.

Proposition A.7 implies that the set of all $f \in \mathfrak{X}_{1}$ with

$$
\operatorname{Hom}_{\mathfrak{g}}(Z(f-a \gamma, \chi, \alpha), Z(f, \chi, \alpha)) \neq 0
$$

is closed in $\mathfrak{X}_{1}$. Proposition F.1 claims that $d \mu$ belongs to this closed set. This will follow when we can show that (2) holds for all $f$ in an open and dense subset of $\mathfrak{X}_{1}$.

Since we assume $p$ to be good, $h_{\alpha}$ and $h_{\gamma}$ are linearly independent in $\mathfrak{h}$. Therefore $\mathfrak{X}_{1}$ is an affine subspace of $\mathfrak{h}^{*}$ of codimension 2 .

If $\beta$ is a root in $\mathbf{Q} \alpha+\mathbf{Q} \gamma$, then $h_{\beta} \in K h_{\alpha}+K h_{\gamma}$ in $\mathfrak{h}$ (since $p$ is good). Each $f \in \mathfrak{X}_{1}$ coincides with $\mu$ (or rather $d \mu$ ) on $h_{\alpha}$ and $h_{\gamma}$, hence on all $h_{\beta}$ with $\beta \in R \cap(\mathbf{Q} \alpha+\mathbf{Q} \gamma)$. We have then $f\left(h_{\beta}\right) \in \mathbf{F}_{p}$ and $\chi(f)\left(h_{\beta}\right)=0$ (recall A.2) for these $\beta$.

On the other hand, if $\beta \in R^{+}$with $\beta \notin \mathbf{Q} \alpha+\mathbf{Q} \gamma$, then $h_{\beta}$ is linearly independent of $h_{\alpha}$ and $h_{\gamma}$ (since $p$ is good). It follows that we can find $f \in \mathfrak{X}_{1}$ with $f\left(h_{\beta}\right) \notin \mathbf{F}_{p}$. Therefore the set

$$
\mathfrak{X}_{1}^{\text {reg }}=\left\{f \in \mathfrak{X}_{1} \mid f\left(h_{\beta}\right) \notin \mathbf{F}_{p} \text { for all } \beta \in R^{+}, \beta \notin \mathbf{Q} \alpha+\mathbf{Q} \gamma\right\}
$$

is open and dense in $\mathfrak{X}_{1}$. By our remarks above, Proposition F.1 will follow from:

Claim. Each $f \in \mathfrak{X}_{1}^{\text {reg }}$ satisfies (2).

Proof (of Claim). Let $f \in \mathfrak{X}_{1}^{\text {reg }}$. The definition of $\mathfrak{X}_{1}^{\text {reg }}$ implies (cf. A.2)

$$
\left\{\beta \in R \mid \chi(f)\left(h_{\beta}\right)=0\right\}=R \cap(\mathbf{Q} \alpha+\mathbf{Q} \gamma) .
$$


We want to apply E.6 and E.7 with $\chi_{0}=\chi(f)$ and $\chi_{1}=\chi$ and $I=\{\alpha\}$. [So the $\chi$ in E.6 is our present $\chi(f)+\chi$.] The Lie subalgebra $\mathfrak{l}$ as in E.7 is by (4) now equal to

$$
\mathfrak{l}=\mathfrak{h} \oplus \bigoplus_{\beta \in R \cap(\mathbf{Q} \alpha+\mathbf{Q} \gamma)} \mathfrak{g}_{\beta}
$$

We get from E.6 a linear form, say $\chi^{\prime}$, on $\mathfrak{g}$ [denoted by $\chi_{1}^{\prime}$ in E.6] with $\chi^{\prime}\left(\mathfrak{p}_{\alpha}\right)=0$ such that $\chi(f)+\chi^{\prime}$ is the Jordan decomposition of some conjugate of $\chi(f)+\chi$. Furthermore E.7 yields an equivalence of categories $\mathcal{F}$. Its description in E.7(1) involves a certain element $\delta_{1} \in X$ satisfying $\delta_{1}\left(h_{\alpha}\right)=0$. The last property implies by A.3(4) for all $\nu \in X$

$$
L_{\chi(f), \alpha}(f+\nu) \otimes \delta_{1} \simeq L_{\chi(f), \alpha}\left(f+\nu+\delta_{1}\right) .
$$

Therefore E.7(1) applied to $M=L_{\chi(f), \alpha}(f+\nu)$ yields

$$
\mathcal{F} Z(f+\nu, \chi, \alpha) \simeq Z_{\chi(f)+\chi^{\prime}}\left(f+\nu+\delta_{1}, \alpha ; \mathfrak{l}\right)
$$

using the notation from A.5.

Note that $d \mu-f$ is a linear form on $\mathfrak{h}$ that vanishes on $K h_{\alpha}+K h_{\gamma}$. So A.4(5) applied to $\mathfrak{l}$ instead of $\mathfrak{g}$ yields

$$
Z_{\chi(f)+\chi^{\prime}}(f+\nu, \alpha ; \mathfrak{l}) \otimes K_{d \mu-f} \simeq Z_{\chi^{\prime}}(\mu+\nu, \alpha ; \mathfrak{l})
$$

for all $\nu \in X$. Set now $\mathcal{F}^{\prime}$ equal to the composition of $\mathcal{F}$ with the functor $N \mapsto$ $N \otimes K_{d \mu-f}$. Then $\mathcal{F}^{\prime}$ is again an equivalence of (appropriate) categories; it satisfies by (6) and (7)

$$
\mathcal{F}^{\prime} Z(f+\nu, \chi, \alpha) \simeq Z_{\chi^{\prime}}\left(\mu+\nu+\delta_{1}, \alpha ; \mathfrak{l}\right) .
$$

So the Hom space in (2) is isomorphic to

$$
\operatorname{Hom}_{\mathfrak{l}}\left(Z_{\chi^{\prime}}\left(\mu-a \gamma+\delta_{1}, \alpha ; \mathfrak{l}\right), Z_{\chi^{\prime}}\left(\mu+\delta_{1}, \alpha ; \mathfrak{l}\right)\right) .
$$

Note that $\mathfrak{l}$ is the Lie algebra of some reductive group satisfying the same assumptions as $G$. Furthermore $\mathfrak{l}$ is either of type $A_{1} \times A_{1}$ or $C_{2}$. So we can apply Lemma F.2 or Lemma F.3 and get

$$
\operatorname{Hom}_{\mathfrak{l}}\left(Z_{\chi^{\prime}}\left(s_{\gamma} \bullet^{\prime} \mu^{\prime}, \alpha ; \mathfrak{l}\right), Z_{\chi^{\prime}}\left(\mu^{\prime}, \alpha ; \mathfrak{l}\right)\right) \neq 0
$$

for all $\mu^{\prime} \in X$. Here $\bullet^{\prime}$ is the dot action for $\mathfrak{l}$, defined as $w \bullet^{\prime} \nu=w\left(\nu+\rho^{\prime}\right)-\rho^{\prime}$ where $\rho^{\prime}$ is half the sum of the positive roots in $R \cap(\mathbf{Q} \alpha+\mathbf{Q} \gamma)$. We have $\left\langle\rho-\rho^{\prime}, \gamma^{\vee}\right\rangle=\left\langle\delta_{1}, \gamma^{\vee}\right\rangle$ by Lemma E.4 and the definition E.5(1). This implies

$$
\begin{aligned}
s_{\gamma} \bullet^{\prime}\left(\mu+\delta_{1}\right) & =\mu+\delta_{1}-\left\langle\mu+\delta_{1}+\rho^{\prime}, \gamma^{\vee}\right\rangle \gamma=\mu+\delta_{1}-\left\langle\mu+\rho, \gamma^{\vee}\right\rangle \gamma \\
& =\left(s_{\gamma} \cdot \mu\right)+\delta_{1} \equiv \mu-a \gamma+\delta_{1} \quad(\bmod p X) .
\end{aligned}
$$

So we can rewrite the first module in (8) as $Z_{\chi^{\prime}}\left(s_{\gamma} \bullet^{\prime}\left(\mu+\delta_{1}\right), \alpha ; \mathfrak{l}\right)$. Now (9) implies that the Hom space in (8) is non-zero. This yields the claim, hence Proposition F.1.

F.5. We now return to the situation from Theorem D.6. So we assume in addition that (D2) holds. Let $\chi \in \mathfrak{g}^{*}$ be subregular nilpotent. We exclude the case where $R$ is of type $G_{2}$; if $R$ is of type $E_{8}$ or $F_{4}$, we assume that $p>h+1$. (The results here as well as in F.6-F.11 hold in these cases also for $p \leq h+1$ provided the socle of $Z_{\chi}(\lambda)$ as in D.4 has the expected dimension.) Recall the definition of the integers $m_{\gamma}$ from D.6(1). 
Theorem. Let $\lambda \in C_{0}^{\prime}$. Let $\gamma$ be a short root with $\gamma \in J(\lambda)$. If $L_{1}$ and $L_{2}$ are composition factors of $Z_{\chi}(\lambda)$ with $\kappa\left(L_{1}\right)=\kappa\left(L_{2}\right)=\{\gamma\}$, then $L_{1} \simeq L_{2}$. If $\chi\left(\mathfrak{b}^{+}\right)=0$, then $L_{1}$ has multiplicity $m_{\gamma}$ as a composition factor of $Z_{\chi}(\lambda)$.

Proof. Lemma B.13 and D.5(3) show: If this theorem holds for one subregular $\chi$, then it holds for all subregular $\chi$. Assume from now on that we choose $\chi$ and the simple root $\alpha$ as in D.12. We get then from Lemma D.12 elements $x_{1}, x_{2} \in W$ with $x_{i} \gamma=\alpha$ and

$$
L_{1} \simeq Z_{\chi}\left(x_{1} \bullet \lambda, \alpha\right), \quad L_{2} \simeq Z_{\chi}\left(x_{2} \bullet \lambda, \alpha\right) .
$$

We have $x_{1} x_{2}^{-1} \alpha=\alpha$. Since $W$ is a reflection group, there are roots $\gamma_{1}, \gamma_{2}, \ldots, \gamma_{r}$ with $x_{1} x_{2}^{-1}=s_{\gamma_{1}} s_{\gamma_{2}} \ldots s_{\gamma_{r}}$ and $s_{\gamma_{i}} \alpha=\alpha$ for all $i$.

Set $y_{i}=s_{\gamma_{i}} s_{\gamma_{i+1}} \ldots s_{\gamma_{r}} x_{2}$ for $1 \leq i \leq r+1$; we get in particular $y_{1}=x_{1}$ and $y_{r+1}=x_{2}$. We have $y_{i}^{-1} \alpha=\gamma$ for all $i$ and $y_{i}=s_{\gamma_{i}} y_{i+1}$ for all $i \leq r$. If we can show that $Z_{\chi}\left(y_{i} \bullet \lambda, \alpha\right)$ and $Z_{\chi}\left(y_{i+1} \bullet \lambda, \alpha\right)$ are isomorphic to each other for all $i \leq r$, then the claim will follow.

This shows that we may assume that there exists a root $\gamma^{\prime}$ orthogonal to $\alpha$ with $x_{2}=s_{\gamma^{\prime}} x_{1}$. Since $\alpha$ is short and since we exclude type $G_{2}$, we can apply Proposition F.1 and get

$$
\operatorname{Hom}_{\mathfrak{g}}\left(Z_{\chi}\left(x_{1} \bullet \lambda, \alpha\right), Z_{\chi}\left(x_{2} \bullet \lambda, \alpha\right)\right) \neq 0 .
$$

This implies that these modules are isomorphic to each other, since they are simple. The claim on $\left[Z_{\chi}(\lambda): L_{1}\right]$ follows now from Theorem D.6.

Remark. Suppose that all roots in $R$ have the same length. Then the theorem says that $\mathcal{C}_{\lambda}$ has $1+|J(\lambda)|$ isomorphism classes of simple modules. For $p$-regular $\lambda$, i.e., if $J(\lambda)$ consists of all simple roots, this confirms a conjecture of Lusztig; see [L2], 14.5, [L4], 2.4, and [L5], 17.2.

F.6. Let $\lambda \in C_{0}^{\prime}$. It is clear that modules in $\mathcal{C}_{\lambda}$ with distinct $\kappa$ invariants are not isomorphic to each other. Given Theorem F.5 the main open problem (besides type $G_{2}$ and the restriction on $p$ in the types $E_{8}$ and $F_{4}$ ) is the classification of the simple modules with $\kappa$ invariant a long simple root. Unfortunately our results are not as complete as in the case treated in F.5.

Again let $\chi$ be subregular. Assume that $R$ has type $B_{n}, C_{n}$, or $F_{4}$; if $R$ is of type $F_{4}$, assume that $p>h+1$.

Proposition. Let $\lambda \in C_{0}^{\prime}$. Let $\gamma$ be a long root with $\gamma \in J(\lambda)$. If $R$ has type $B_{n}$, then there are two isomorphism classes of simple modules with $\kappa$ invariant $\{\gamma\}$. In the other cases there are at most two such isomorphism classes.

Proof. As in F.5 we get: If this theorem holds for one subregular $\chi$, then it holds for all subregular $\chi$.

If $R$ is of type $B_{n}$, then we assume that $\chi$ has the form as in [J2], Section 3 . In this case the claim follows from the results in [J2]: If $\gamma=\alpha_{i}$, then the two isomorphism classes are represented by $L_{\chi}\left(\lambda_{i}\right)$ and $L_{\chi}\left(\lambda_{2 n-i}\right)$ in the notation from [J2].

Assume now that $R$ has type $C_{n}$. Then $\gamma$ has to be equal to $\alpha_{n}$. We may assume that $\chi\left(\mathfrak{p}_{\alpha_{n}}\right)=0$. Set

$$
W_{1}=\left\{w \in W \mid w \alpha_{n}=\alpha_{n}\right\} .
$$


Recall that all $Z_{\chi}\left(x \bullet \lambda, \alpha_{n}\right)$ with $x \in W_{1}$ are simple with $\kappa$ invariant $\left\{\alpha_{n}\right\}$; see D.3.a and D.5.c. On the other hand, each simple module $L$ in $\mathcal{C}_{\lambda}$ with $\kappa(L)=\left\{\alpha_{n}\right\}$ is by D.13 isomorphic to some $Z_{\chi}\left(x \bullet \lambda, \alpha_{n}\right)$ with $x \in W_{1}$.

Since $W_{1}$ is generated by all $s_{\beta}$ with $\beta$ orthogonal to $\alpha_{n}=2 \varepsilon_{n}$, it is clear that $W_{1}$ is the Weyl group of the root system $R_{1}=R \cap \sum_{i<n} \mathbf{Q} \varepsilon_{i}$. This is a root system of type $C_{n-1}$. The short roots in $R_{1}$ are a root system $R_{2}$ of type $D_{n-1}$; denote the corresponding Weyl group by $W_{2}$. Then $W_{2}$ has index 2 in $W_{1}$ and we have

$$
W_{1}=W_{2} \cup W_{2} s_{2 \varepsilon_{n-1}} \text {. }
$$

If $\gamma$ is in $R_{2}$, then $R \cap\left(\mathbf{Q} \alpha_{n}+\mathbf{Q} \gamma\right)$ is of type $A_{1} \times A_{1}$. Then Proposition F.1 implies for all $x \in W_{1}$ that $Z_{\chi}\left(x \bullet \lambda, \alpha_{n}\right)$ is isomorphic to $Z_{\chi}\left(s_{\gamma} x \bullet \lambda, \alpha_{n}\right)$. So (1) shows for all $x \in W_{1}$ that

$$
Z_{\chi}\left(x \bullet \lambda, \alpha_{n}\right) \simeq \begin{cases}Z_{\chi}\left(\lambda, \alpha_{n}\right), & \text { if } x \in W_{2} ; \\ Z_{\chi}\left(s_{2 \varepsilon_{n-1}} \bullet \lambda, \alpha_{n}\right), & \text { if } x \notin W_{2} .\end{cases}
$$

So there are two possibilities: Either all $Z_{\chi}\left(x \bullet \lambda, \alpha_{n}\right)$ are isomorphic to $Z_{\chi}\left(\lambda, \alpha_{n}\right)$, or these modules fall into two isomorphism classes.

Consider finally $R$ of type $F_{4}$. We may assume that $\chi\left(\mathfrak{p}_{\alpha_{1}}\right)=0$. The set of roots orthogonal to $\alpha_{1}$ is a root system of type $C_{3}$. (Note that $\alpha_{1}$ is conjugate to the largest root, i.e., to $\varpi_{1}$.) A basis of this root system is $\alpha_{4}, \alpha_{3}, \varepsilon_{2}+\varepsilon_{3}=$ $\alpha_{1}+2 \alpha_{2}+2 \alpha_{3}$. The stabiliser $W_{1}$ of $\alpha_{1}$ is the Weyl group of this subsystem. The short roots orthogonal to $\alpha_{1}$ form a root system of type $D_{3}=A_{3}$. The corresponding Weyl group $W_{2}$ is a subgroup of index 2 in $W_{1}$; we have

$$
W_{1}=W_{2} \cup W_{2} s_{\varepsilon_{2}+\varepsilon_{3}} .
$$

We have $\gamma \in\left\{\alpha_{1}, \alpha_{2}\right\}$. The simple modules in $\mathcal{C}_{\lambda}$ with $\kappa$ invariant $\{\gamma\}$ are (by D.3.a, D.5.c and D.13) the $Z_{\chi}\left(x \bullet \lambda, \alpha_{1}\right)$ with $x \in W$ and $x \gamma=\alpha_{1}$. The $x$ with this property are a coset for $W_{1}$, in fact equal to $W_{1}$ in case $\gamma=\alpha_{1}$, equal to $W_{1} s_{2} s_{1}$ in case $\gamma=\alpha_{2}$. Proposition F.1 implies for all $w \in W_{2}$ (and $x$ as above) that $Z_{\chi}\left(w x \bullet \lambda, \alpha_{1}\right) \simeq Z_{\chi}\left(x \bullet \lambda, \alpha_{1}\right)$. This yields for all $x \in W_{1}$ in case $\gamma=\alpha_{1}$

$$
Z_{\chi}\left(x \cdot \lambda, \alpha_{1}\right) \simeq \begin{cases}Z_{\chi}\left(\lambda, \alpha_{1}\right), & \text { if } x \in W_{2}, \\ Z_{\chi}\left(s_{\varepsilon_{2}+\varepsilon_{3}} \bullet \lambda, \alpha_{1}\right), & \text { if } x \notin W_{2},\end{cases}
$$

and in case $\gamma=\alpha_{2}$

$$
Z_{\chi}\left(x s_{2} s_{1} \bullet \lambda, \alpha_{1}\right) \simeq \begin{cases}Z_{\chi}\left(s_{2} s_{1} \bullet \lambda, \alpha_{1}\right), & \text { if } x \in W_{2}, \\ Z_{\chi}\left(s_{\varepsilon_{2}+\varepsilon_{3}} s_{2} s_{1} \bullet \lambda, \alpha_{1}\right), & \text { if } x \notin W_{2} .\end{cases}
$$

Remark. Lusztig's conjectures predict that we should have two classes also for types $C_{n}$ and $F_{4}$.

F.7. Given $\chi \in \mathfrak{g}^{*}$ each $g \in C_{G}(\chi)$ (the stabiliser of $\chi$ under the coadjoint action) permutes the isomorphism classes of simple $U_{\chi}(\mathfrak{g})$-modules via $L \mapsto{ }^{g} L$. Since there are only finitely many classes, one sees easily that $C_{G}(\chi)^{0}$, the connected component of the identity in $C_{G}(\chi)$, acts trivially. So does the centre $Z(G)$. This means that we are really looking at an action of the "component group" $A(\chi)=$ $C_{G}(\chi) /\left(Z(G) C_{G}(\chi)^{0}\right)$. (It is really the component group for the adjoint group.)

Note that this action is "the same" for all $\chi$ in a fixed $G$-orbit: Given $h \in G$ we have $C_{G}(h \chi)=h C_{G}(\chi) h^{-1}$; therefore conjugation with $h$ induces an isomorphism $A(\chi) \stackrel{\sim}{\longrightarrow} A(h \chi)$. Furthermore, the map $L \mapsto{ }^{h} L$ induces a bijection from the set of 
isomorphism classes of simple $U_{\chi}(\mathfrak{g})$-modules to the corresponding set for $U_{h \chi}(\mathfrak{g})$. This bijection is compatible with the actions of $A(\chi)$ and $A(h \chi)$ identified as above.

We have used before that $U(\mathfrak{g})^{G}$ acts on ${ }^{g} L$ as it does on $L$. Therefore the action of $A(\chi)$ permutes (for $\chi$ nilpotent) the simple modules in each $\mathcal{C}_{\lambda}$. (For general $\chi$ one would have to introduce a new notation.) By D.5(3) the action of $A(\chi)$ preserves the $\kappa$ invariant (when defined).

Suppose now that $\chi$ is subregular and nilpotent. If all roots have the same length, then F.5 and the remarks above show that $A(\chi)$ acts trivially. Actually, we have in these cases $A(\chi)=1$ at least for large $p$; see [Sl], 7.5.

In the next subsections we are going to prove:

Proposition. Suppose that $R$ has type $B_{n}, C_{n}$, or $F_{4}$; if $R$ has type $F_{4}$, assume that $p>h+1$. Let $\lambda \in C_{0}^{\prime}$, let $\gamma$ be a long root with $\gamma \in J(\lambda)$. If $\chi$ is subregular, then $A(\chi)$ permutes transitively the isomorphism classes of simple modules in $\mathcal{C}_{\lambda}$ with $\kappa$ invariant $\{\gamma\}$.

Remarks. 1) We have $A(\chi) \simeq \mathbf{Z} / 2 \mathbf{Z}$ for the groups considered in the proposition, at least if $p$ is large; see [Sl], 7.5. (In types $B_{n}$ and $C_{n}$ it suffices to assume that $p>2$; see [SpSt], IV.2.26.)

2) The claim is of course trivial in the (unexpected) case that there is only one isomorphism class of such modules. If however there are two and if $L$ and $L^{\prime}$ are representatives for these classes, then the proposition implies [by B.13(3)] that $L$ and $L^{\prime}$ occur with the same multiplicity in $Z_{\chi}(\lambda)$ in case $\chi\left(\mathfrak{b}^{+}\right)=0$. That multiplicity then has to be equal to $m_{\gamma} / 2$ in the notation from D.6(1).

F.8. We look first at type $B_{n}$. I shall now use freely the notations and assumptions from [J2], Section 3. In particular, we have $\chi$ subregular with $\chi\left(x_{-\beta}\right) \neq 0$ if and only if $\beta \in\left\{\alpha_{2}, \alpha_{3}, \ldots, \alpha_{n}\right\}$. We work with an arbitrary $\lambda \in C_{0}$.

We can find in $N_{G}(T)$ a representative $g$ of $s_{\varepsilon_{1}}$ with $g \chi=\chi$. (Each representative $g$ will satisfy $\operatorname{Ad}(g) x_{-\alpha_{i}} \in K x_{-\alpha_{i}}$ for all $i>1$ since $s_{\varepsilon_{1}} \alpha_{i}=\alpha_{i}$. Multiplying $g$ with a suitable element in $T$ we can get $\operatorname{Ad}(g) x_{-\alpha_{i}}=x_{-\alpha_{i}}$ for all $i>1$. Then $g \chi=\chi$ holds.)

Claim. Suppose that $1 \leq i \leq 2 n$ with $b_{i}<b_{i+1}$. Then

$$
{ }^{g} L_{\chi}\left(\lambda_{i}\right) \simeq \begin{cases}L_{\chi}\left(\lambda_{2 n-i}\right) \otimes 4(n-1) p, & \text { if } i<2 n \\ L_{\chi}\left(\lambda_{2 n}\right) \otimes(4 n-2) p, & \text { if } i=2 n\end{cases}
$$

as $U_{\chi}(\mathfrak{g})-T_{0}-$ modules.

Proof. Recall that $T_{0}$ is the one parameter subgroup of $T$ corresponding to $2 \varepsilon_{1}$; see [J2], 3.2. This implies that $g t g^{-1}=t^{-1}$ for all $t \in T_{0}$. One checks easily that the Lie subalgebra $\mathfrak{m}$ from [J2], 3.4 is stable under $\operatorname{Ad}(g)$. It follows that

$$
\left({ }^{g} M\right)^{\mathfrak{m}}={ }^{g}\left(M^{\mathfrak{m}}\right)
$$

for each $U_{\chi}(\mathfrak{g})-T_{0}$-module $M$. Furthermore the weights of $T_{0}$ on $\left({ }^{g} M\right)^{\mathfrak{m}}$ are the negatives of the weights of $T_{0}$ on $M^{\mathfrak{m}}$.

Apply this to $M=L_{\chi}\left(\lambda_{i}\right)$. We know by [J2], 3.8 that ${ }^{g} L_{\chi}\left(\lambda_{i}\right)$ is isomorphic to some $L_{\chi}\left(\lambda_{l}\right) \otimes m p$ with $l, m \in \mathbf{Z}$ and $1 \leq l \leq 2 n$. We can determine $l$ and $m$ using the weights of $T_{0}$ on $\left({ }^{g} L_{\chi}\left(\lambda_{i}\right)\right)^{\mathfrak{m}}$. The necessary calculations are the same ones that prove [J2], 3.14(5), but were not written up in [J2]. 
The weights of $T_{0}$ on $L_{\chi}\left(\lambda_{i}\right)^{\mathfrak{m}}$ are by [J2], 3.10(3) and 3.6(6) all

$$
\left\langle\lambda_{1}, \psi\right\rangle-2(n-1)(p-1)-2 j \quad \text { with } b_{i} \leq j<b_{i+1} .
$$

So the weights on $\left({ }^{g} L_{\chi}\left(\lambda_{i}\right)\right)^{\mathfrak{m}}$ are all

$$
-\left\langle\lambda_{1}, \psi\right\rangle+2(n-1)(p-1)+2 j=\left\langle\lambda_{1}, \psi\right\rangle-2(n-1)(p-1)-2 j^{\prime}+4(n-1) p
$$

with $j$ as above and

$$
j^{\prime}=\left\langle\lambda_{1}, \psi\right\rangle+2(n-1)-j .
$$

We have $b_{2 n}=\left\langle\lambda_{1}, \psi\right\rangle+2 n-1$ and $b_{i}+b_{2 n+1-i}=b_{2 n}$ for $1 \leq i \leq 2 n$; see [J2], $3.2(1)$ and 3.6(8). If $j$ runs from $b_{i}$ to $b_{i+1}-1$ (and if $i<2 n$ ), then $j^{\prime}=b_{2 n}-j$ runs from $b_{2 n-i}$ to $b_{2 n+1-i}-1$. This yields the claim for $i<2 n$. In case $i=2 n$ recall that $b_{2 n+1}=p$; so now $j$ runs from $b_{2 n}$ to $p-1$, hence $j^{\prime}$ from $b_{2 n}-p$ to -1 , and $j^{\prime}+p$ from $b_{2 n}$ to $p-1$. This yields the claim in that case.

F.9. Keep for the moment the notation from F.8. Suppose that $\lambda \in C_{0}^{\prime}$. If $b_{i}<b_{i+1}$ for some $i<2 n$, then the simple module $L_{\chi}\left(\lambda_{i}\right)$ has $\kappa$ invariant $\left\{\alpha_{i}\right\}$ if $i \leq n$, resp. $\left\{\alpha_{2 n-i}\right\}$ if $i>n$. This follows, e.g., from [J2], 3.13. Furthermore, $L_{\chi}\left(\lambda_{2 n}\right)$ is the socle of $Z_{\chi}\left(\lambda_{1}\right)$.

Now Proposition F.7 follows for $R$ of type $B_{n}$ immediately from the claim in F.8. (We also see that $g$ has to be a representative for the non-trivial class in $A(\chi)$. That, however, follows also from the descriptions in [SpSt] or [Sl].)

With a view to another application let me look a bit more precisely at the case $n=2$. Consider $\chi$ as in F.8. Given $\lambda_{1} \in C_{0}$ with $\left\langle\lambda_{1}+\rho, \alpha_{1}^{\vee}\right\rangle>0$, then [J2], 3.13 says that

$$
L_{\chi}\left(\lambda_{1}\right) \simeq Z_{\chi}\left(\lambda_{1}, \alpha_{1}\right) \quad \text { and } \quad L_{\chi}\left(\lambda_{3}\right) \simeq Z_{\chi}\left(s_{\varepsilon_{1}+\varepsilon_{2}} \cdot \lambda_{1}, \alpha_{1}\right) .
$$

So Claim F.8 says that

$$
{ }^{g} Z_{\chi}\left(\lambda_{1}, \alpha_{1}\right) \simeq Z_{\chi}\left(s_{\varepsilon_{1}+\varepsilon_{2}} \bullet \lambda_{1}, \alpha_{1}\right)
$$

It will be convenient to change notation and assume that $R$ is of type $C_{2}$. Now let $\chi \in \mathfrak{g}^{*}$ have standard Levi form with $\chi\left(x_{-\beta}\right) \neq 0$ if and only if $\beta=\alpha_{1}$. Let $g$ be a representative in $N_{G}(T)$ of $s_{\varepsilon_{1}+\varepsilon_{2}}$ with $g \chi=\chi$. Then we have for all $\lambda \in C_{0}$ with $\left\langle\lambda+\rho, \alpha_{2}^{\vee}\right\rangle>0$

$$
{ }^{g} Z_{\chi}\left(\lambda, \alpha_{2}\right) \simeq Z_{\chi}\left(s_{2 \varepsilon_{1}} \bullet \lambda, \alpha_{2}\right) .
$$

This is an immediate translation of (1).

F.10. Consider $R$ of type $C_{n}$. One can check that

$$
x=\sum_{i=1}^{n-1} x_{\alpha_{i}}+x_{\alpha_{n-2}+\alpha_{n-1}+\alpha_{n}}
$$

is subregular. [It acts for the natural representation on $K^{2 n}$ with partition $(2 n-2,2)$.] So the corresponding (under $\left.\mathfrak{g} \stackrel{\sim}{\longrightarrow} \mathfrak{g}^{*}\right)$ linear form $\chi$ satisfies $\chi\left(\mathfrak{p}_{\alpha_{n}}\right)=0$ and $\chi\left(x_{-\alpha_{i}}\right) \neq 0$ for all $i<n$.

Choose a representative $g \in N_{G}(T)$ of $s_{\varepsilon_{n-1}+\varepsilon_{n}}$ such that $g \chi=\chi$. (This is possible, more or less as in F.8: If $g$ is an arbitrary representative, then $\operatorname{Ad}(g) x_{-\alpha_{i}} \in$ $K x_{-\alpha_{i}}$ for $i<n-2$ and $i=n-1$ while $\operatorname{Ad}(g) x_{-\alpha_{n-2}} \in K x_{-\left(\varepsilon_{n-2}+\varepsilon_{n}\right)}$ and $\operatorname{Ad}(g) x_{-\left(\varepsilon_{n-2}+\varepsilon_{n}\right)} \in K x_{-\alpha_{n-2}}$. Now multiply $g$ by a suitable element in $T$.) Proposition F.7 follows in this case from F.6(2) and: 
Claim. We have

$$
{ }^{g} Z_{\chi}\left(\lambda, \alpha_{n}\right) \simeq Z_{\chi}\left(s_{2 \varepsilon_{n-1}} \bullet \lambda, \alpha_{n}\right)
$$

for all $\lambda \in C_{0}$ with $\left\langle\lambda+\rho, \alpha_{n}^{\vee}\right\rangle>0$.

Proof. Set $J=\left\{\alpha_{n-1}, \alpha_{n}\right\}$ and use the abbreviation

$$
Z_{\chi, J}\left(\mu, \alpha_{n}\right)=U_{\chi}\left(\mathfrak{p}_{J}\right) \otimes_{U_{\chi}\left(\mathfrak{p}_{\alpha_{n}}\right)} L_{\chi, \alpha_{n}}(\mu)
$$

for all $\mu \in X$. We have by transitivity of induction

$$
Z_{\chi}\left(\mu, \alpha_{n}\right) \simeq U_{\chi}(\mathfrak{g}) \otimes_{U_{\chi}\left(\mathfrak{p}_{J}\right)} Z_{\chi, J}\left(\mu, \alpha_{n}\right)
$$

for all $\mu$. Note that $g$ belongs to the standard Levi factor $G_{J}$ of $P_{J}$ since we can find there at least one representative of $s_{\varepsilon_{n-1}+\varepsilon_{n}}$ [coming from the root subgroups $\left.U_{ \pm\left(\varepsilon_{n-1}+\varepsilon_{n}\right)}\right]$. Therefore we have for all $\mu$

$$
{ }^{g} Z_{\chi}\left(\mu, \alpha_{n}\right) \simeq U_{\chi}(\mathfrak{g}) \otimes_{U_{\chi}\left(\mathfrak{p}_{J}\right)}{ }^{g} Z_{\chi, J}\left(\mu, \alpha_{n}\right) ;
$$

cf. B.14(1). So our claim will follow if we can show that

$$
{ }^{g} Z_{\chi, J}\left(\lambda, \alpha_{n}\right) \simeq Z_{\chi, J}\left(s_{2 \varepsilon_{n-1}} \cdot \lambda, \alpha_{n}\right) .
$$

The nilradical of $\mathfrak{p}_{J}$ acts as 0 on both sides in (2). So we just have to find an isomorphism of $\mathfrak{g}_{J}$-modules. The centre of $\mathfrak{g}_{J}$ [equal to the intersection in $\mathfrak{h}$ of $\operatorname{ker}\left(\alpha_{n}\right)$ and $\left.\operatorname{ker}\left(\alpha_{n-1}\right)\right]$ acts on both sides via the restriction of $\lambda$. So we just have to find an isomorphism as modules over the derived Lie algebra $\mathcal{D} \mathfrak{g}_{J}$ of $\mathfrak{g}_{J}$.

We want to apply F.9(2) to $\mathcal{D} \mathfrak{g}_{J}$ in order to get the isomorphism in (2) and thus the claim. The restriction of $\chi$ to $\mathcal{D} \mathfrak{g}_{J}$ has standard Levi form. We have $G_{J}=Z\left(G_{J}\right)^{0} \mathcal{D} G_{J}$ [where $Z\left(G_{J}\right)^{0} \subset T$ is the connected centre] and can thus write $g=z g^{\prime}$ with $z \in Z\left(G_{J}\right)^{0}$. Since $z$ trivially fixes $\chi$ on $\mathcal{D} \mathfrak{g}_{J}$, so does $g^{\prime}$. Since $g^{\prime}$ is still a representative for $s_{\varepsilon_{n-1}+\varepsilon_{n}}$, we can now apply F.9(2) and get the claim.

F.11. Suppose now that $R$ is of type $F_{4}$. Set

$$
x=x_{\alpha_{2}}+x_{\alpha_{3}}+x_{\alpha_{4}}+x_{\alpha_{1}+\alpha_{2}+\alpha_{3}+\alpha_{4}} .
$$

Let $\chi$ be the linear form corresponding to $x$. Then $\chi$ is subregular (see the end of the proof of D.13) with $\chi\left(\mathfrak{p}_{\alpha_{1}}\right)=0$. We can find a representative $g \in N_{G}(T)$ for the reflection $s_{\varepsilon_{2}}=s_{\alpha_{1}+\alpha_{2}+\alpha_{3}}$ such that $g \chi=\chi$. Proposition F.7 follows in this case from F.6(4),(5) and:

Claim. Let $\lambda \in C_{0}$. We have

$$
{ }^{g} Z_{\chi}\left(\lambda, \alpha_{1}\right) \simeq Z_{\chi}\left(s_{\varepsilon_{2}+\varepsilon_{3}} \cdot \lambda, \alpha_{1}\right)
$$

if $\left\langle\lambda+\rho, \alpha_{1}^{\vee}\right\rangle>0$, and

$$
{ }^{g} Z_{\chi}\left(s_{2} s_{1} \bullet \lambda, \alpha_{1}\right) \simeq Z_{\chi}\left(s_{\varepsilon_{2}+\varepsilon_{3}} s_{2} s_{1} \bullet \lambda, \alpha_{1}\right)
$$

if $\left\langle\lambda+\rho, \alpha_{2}^{\vee}\right\rangle>0$.

Proof. Note that $\varepsilon_{2}+\varepsilon_{3}=\alpha_{1}+2 \alpha_{2}+2 \alpha_{3} \in R$. Set $J=\left\{\alpha_{1}, \alpha_{2}, \alpha_{3}\right\}$; we have then $g \in G_{J}$. We basically proceed as in F.10 using an abbreviation $Z_{\chi, J}\left(\mu, \alpha_{1}\right)$ as there. We have to show

$$
{ }^{g} Z_{\chi, J}\left(\lambda, \alpha_{1}\right) \simeq Z_{\chi, J}\left(s_{\varepsilon_{2}+\varepsilon_{3}} \bullet \lambda, \alpha_{1}\right)
$$

and

$$
{ }^{g} Z_{\chi, J}\left(s_{2} s_{1} \bullet \lambda, \alpha_{1}\right) \simeq Z_{\chi, J}\left(s_{\varepsilon_{2}+\varepsilon_{3}} s_{2} s_{1} \bullet \lambda, \alpha_{1}\right) .
$$


Again, it suffices to find isomorphisms of $\mathcal{D} \mathfrak{g}_{J}$-modules. Now $\mathcal{D} \mathfrak{g}_{J}$ has type $B_{3}$. We now apply F.8 with $n=3$ and get (4) from the case $i=1$ and (5) from $i=2$. Well, we have to take another look at [J2], 3.13 to check that we can choose there $\lambda_{2}=s_{2} s_{1} \bullet \lambda_{1}$ and $\lambda_{4}=s_{\varepsilon_{1}+\varepsilon_{2}} s_{2} s_{1} \bullet \lambda_{1}$ and $\lambda_{5}=s_{\varepsilon_{1}+\varepsilon_{2}} \bullet \lambda_{1}$.

\section{G.}

We keep the assumptions from Section D. However, in G.2 and G.3 only (D1) is needed.

G.1. Assume that $\chi \in \mathfrak{g}^{*}$ is subregular nilpotent. We exclude $R$ of type $G_{2}$, and assume that $p>h+1$, if $R$ has type $E_{8}$ or $F_{4}$. Given $\lambda \in C_{0}^{\prime}$ we write $L_{0}^{\lambda}$ for the simple module in $\mathcal{C}_{\lambda}$ with $\kappa$ invariant $J(\lambda) \cup\{0\}$. If $\beta \in J(\lambda)$ and if there is up to isomorphism only one simple module in $\mathcal{C}_{\lambda}$ with $\kappa$ invariant $\{\beta\}$, then we denote such a simple module by $L_{\beta}^{\lambda}$. If there are two such modules (up to isomorphism), then we denote them by $L_{\beta, 1}^{\lambda}$ and $L_{\beta, 2}^{\lambda}$.

Lemma. Let $\lambda, \mu \in C_{0}^{\prime}$ such that $\mu$ is in the closure of the facet of $\lambda$.

a) We have $T_{\lambda}^{\mu} L_{0}^{\lambda} \simeq L_{0}^{\mu}$.

b) Let $\beta \in J(\lambda)$. If $L$ is a simple module in $\mathcal{C}_{\lambda}$ with $\kappa(L)=\{\beta\}$, then $T_{\lambda}^{\mu} L=0$ if $\beta \notin J(\mu)$, while $T_{\lambda}^{\mu} L$ is a simple module with $\kappa\left(T_{\lambda}^{\mu} L\right)=\{\beta\}$ if $\beta \in J(\mu)$.

Proof. By B.13(2) and D.5(3) the claim holds for all $\chi$, if it holds for one $\chi$. So we may assume that $\chi\left(\mathfrak{b}^{+}\right)=0$. Then the claim in a) follows from

$$
T_{\lambda}^{\mu} L_{0}^{\lambda}=T_{\lambda}^{\mu} \operatorname{sbm}\left(w_{0}, \lambda\right) \simeq \operatorname{sbm}\left(w_{0}, \mu\right) \simeq \operatorname{soc} Z_{\chi}(\mu),
$$

see C.9(1) and C.10(2).

In order to prove b) we may assume that $\chi$ has the form considered in D.12 or in D.13, hence that $L \simeq Z_{\chi}(x \bullet \lambda, \alpha)$ with $\alpha$ as in D.12 and D.13 and $x \in W$ such that $x \beta=\alpha$. Now the claim follows from B.11 and D.3.a.

Remark. If $\beta$ is short, then we can express the claim in b) as

$$
T_{\lambda}^{\mu} L_{\beta}^{\lambda} \simeq \begin{cases}L_{\beta}^{\mu}, & \text { if } \beta \in J(\mu), \\ 0, & \text { if } \beta \notin J(\mu) .\end{cases}
$$

For long $\beta$ things get more complicated, in particular when we do not know the number of simple modules with a given $\kappa$ invariant.

G.2. Now let $\chi$ be an arbitrary linear form on $\mathfrak{g}$ with $\chi\left(\mathfrak{b}^{+}\right)=0$.

Lemma. Each projective $U_{\chi}(\mathfrak{g})$-module is a direct summand of some $E \otimes Z_{\chi}(-\rho)$ with $E$ a $G$-module.

Proof. It suffices to look at the projective cover $Q_{L}$ of a simple $U_{\chi}(\mathfrak{g})$-module $L$. There exists a weight $\mu \in X$ such that $L$ is a homomorphic image of $Z_{\chi}(\mu)$. In fact, we can find a dominant weight $\nu$ such that $L$ is a homomorphic image of $Z_{\chi}\left(-\rho+w_{0} \nu\right)$ since $Z_{\chi}(\mu)$ depends only on $\mu+p X$ and since each coset in $X / p X$ has a representative of the form $-\rho+w_{0} \nu$ as above. Now take $E$ as the simple module with highest weight $\nu$. Then $E \otimes Z_{\chi}(-\rho)$ has a filtration with factors $Z_{\chi}\left(-\rho+\nu^{\prime}\right)$ with $\nu^{\prime}$ running over the weights of $L$. The factor $Z_{\chi}\left(-\rho+w_{0} \nu\right)$ occurs thus as a homomorphic image of $E \otimes Z_{\chi}(-\rho)$. So does then $L$. Because $E \otimes Z_{\chi}(-\rho)$ is projective, this implies that $Q_{L}$ is a direct summand of $E \otimes Z_{\chi}(-\rho)$. 
G.3. Let $\chi$ be as in G.2. We say that a $U_{\chi}(\mathfrak{g})$-module $M$ has a $Z$-filtration if there exists a chain of submodules in $M$, beginning with 0 and ending with $M$, such that all subsequent factors are isomorphic to some $Z_{\chi}(\nu)$ with $\nu \in X$. If so, then also each $E \otimes M$ with $E$ a $G$-module, and each $\operatorname{pr}_{\mu}(M)$ with $\mu \in X$ has a $Z$-filtration; cf. B.3. Therefore all modules of the form

$$
\operatorname{pr}_{\mu_{r}}\left(E_{r} \otimes \operatorname{pr}_{\mu_{r-1}}\left(E_{r-1} \otimes \cdots \operatorname{pr}_{\mu_{2}}\left(E_{2} \otimes \operatorname{pr}_{\mu_{1}}\left(E_{1} \otimes Z_{\chi}(-\rho)\right)\right) \ldots\right)\right)
$$

have a $Z$-filtration. All these modules are projective, since $Z_{\chi}(-\rho)$ is projective; see [FP2], Thm. 4.1.

Set $\mathcal{P}$ equal to the Grothendieck group of all projective $U_{\chi}(\mathfrak{g})$-modules. So this is a free Abelian group with the projective covers of the simple $U_{\chi}(\mathfrak{g})$-modules as a basis. We shall usually write $Q$ also for the class in $\mathcal{P}$ of a projective $U_{\chi}(\mathfrak{g})$-module $Q$.

Let $\mathcal{P}^{\prime}$ denote the subgroup of $\mathcal{P}$ generated by all modules as in (1). For each $\lambda \in X$ let $\mathcal{P}_{\lambda}$ be the Grothendieck group of all projective modules in $\mathcal{C}_{\lambda}$, and set $\mathcal{P}_{\lambda}^{\prime}=\mathcal{P}^{\prime} \cap \mathcal{P}_{\lambda}$. Then $\mathcal{P}$ is the direct sum of all $\mathcal{P}_{\lambda}$ (and $\mathcal{P}^{\prime}$ that of all $\mathcal{P}_{\lambda}^{\prime}$ ) with $\lambda$ running over a suitable set of representatives. It is clear that

$$
T_{\lambda}^{\mu} \mathcal{P}_{\lambda}^{\prime} \subset \mathcal{P}_{\mu}^{\prime}
$$

for all $\lambda, \mu \in X$.

Lemma. Let $\lambda \in X$, let $Q$ be a projective module in $\mathcal{C}_{\lambda}$. If there exists an integer $m>0$ with $Q^{m} \in \mathcal{P}_{\lambda}^{\prime}$, then we have

$$
[Q]=\frac{\operatorname{dim} Q}{p^{N}}\left[Z_{\chi}(\lambda)\right]
$$

in the Grothendieck group of all modules in $\mathcal{C}_{\lambda}$.

Proof. Since this Grothendieck group is free over $\mathbf{Z}$, it suffices to prove the claim for $Q^{m}$. So we may assume that $Q \in \mathcal{P}_{\lambda}^{\prime}$. So $Q$ is a $\mathbf{Z}$-linear combination (in $\mathcal{P}^{\prime}$ ) of modules as in (1) [with $\mu_{r}=\lambda$ ]. Since the claim in (3) is additive in $Q$, we may assume that $Q$ is a module as in (1), hence that $Q$ has a $Z$-filtration. But then the claim follows from the fact that $\left[Z_{\chi}(w \bullet \lambda)\right]=\left[Z_{\chi}(\lambda)\right]$ for all $w \in W$.

Remark. Let $L$ be a simple module in $\mathcal{C}_{\lambda}$. Suppose that the projective cover $Q_{L}$ of $L$ satisfies the assumption of our lemma. Then (3) and B.12(2) imply

$$
\left[Q_{L}\right]=|W \cdot(\lambda+p X)|\left[Z_{\chi}(\lambda): L\right]\left[Z_{\chi}(\lambda)\right],
$$

hence

$$
\left[Q_{L}: L^{\prime}\right]=|W \cdot(\lambda+p X)|\left[Z_{\chi}(\lambda): L\right]\left[Z_{\chi}(\lambda): L^{\prime}\right]
$$

for all simple $L^{\prime}$ in $\mathcal{C}_{\lambda}$.

G.4. We return to the assumptions and conventions from G.1. In particular, we have $\chi \in \mathfrak{g}^{*}$ subregular nilpotent. Given $\lambda \in C_{0}^{\prime}$ we denote by $Q_{0}^{\lambda}$ (resp. $Q_{\beta}^{\lambda}$ or $Q_{\beta, i}^{\lambda}$ ) the projective cover in $\mathcal{C}_{\lambda}$ of the simple module $L_{0}^{\lambda}$ (resp. $L_{\beta}^{\lambda}$ or $L_{\beta, i}^{\lambda}$ ).

Proposition C.2 says that $Q_{0}^{\lambda} \simeq T_{-\rho}^{\lambda} Z_{\chi}(-\rho)$. So we get

$$
Q_{0}^{\lambda} \in \mathcal{P}_{\lambda}^{\prime}
$$

for all $\lambda \in C_{0}^{\prime}$. 
Lemma. Let $\alpha$ be a short simple root. Then there exists an integer $n(\alpha)>0$ such that

$$
n(\alpha) Q_{\alpha}^{\varpi_{\alpha}-\rho} \in \mathcal{P}_{\varpi_{\alpha}-\rho}^{\prime} .
$$

Proof. Set $\mu=\varpi_{\alpha}-\rho$. There are in $\mathcal{C}_{\mu}$ (up to isomorphism) only two simple modules: $L_{\alpha}^{\mu}$ and $L_{0}^{\mu}$, hence only two indecomposable projective modules: $Q_{\alpha}^{\mu}$ and $Q_{0}^{\mu}$. Lemma G.2 yields a $G$-module $E$ such that $Q_{\alpha}^{\mu}$ is a direct summand of $E \otimes Z_{\chi}(-\rho)$. Then there exist integers $n(\alpha)>0$ and $m \geq 0$ with

$$
\operatorname{pr}_{\mu}\left(E \otimes Z_{\chi}(-\rho)\right) \simeq\left(Q_{\alpha}^{\mu}\right)^{n(\alpha)} \oplus\left(Q_{0}^{\mu}\right)^{m} .
$$

Now the definition of $\mathcal{P}^{\prime}$ and (1) yield the claim.

Remark. Let $\alpha$ be a long simple root. Set $\mu=\varpi_{\alpha}-\rho$. Should (against expectations) there be only one simple module in $\mathcal{C}_{\mu}$ with $\kappa$ invariant $\{\alpha\}$, then we get as above an integer $n(\alpha)>0$ with $n(\alpha) Q_{\alpha}^{\mu} \in \mathcal{P}_{\mu}^{\prime}$. If however, as expected, there are two such modules, then we get instead

$$
n(\alpha)\left(Q_{\alpha, 1}^{\mu}+Q_{\alpha, 2}^{\mu}\right) \in \mathcal{P}_{\mu}^{\prime} .
$$

Indeed, we have in this situation by Proposition F.7 an element $g \in G$ with $g \chi=$ $\chi$ and ${ }^{g} L_{\alpha, 1}^{\mu} \simeq L_{\alpha, 2}^{\mu}$, hence ${ }^{g} Q_{\alpha, 1}^{\mu} \simeq Q_{\alpha, 2}^{\mu}$. On the other hand, we have above ${ }^{g} E \simeq E$ [since this is a $G$-module] and ${ }^{g} Z_{\chi}(-\rho) \simeq Z_{\chi}(-\rho)$ [since this is the only simple module in $\mathcal{C}_{-} \rho$ ]. Therefore the multiplicities of $Q_{\alpha, 1}^{\mu}$ and of ${ }^{g} Q_{\alpha, 1}^{\mu}$ as direct summands of $E \otimes Z_{\chi}(-\rho)$ have to be equal. (The same argument shows that ${ }^{g} Q \simeq Q$ for all $Q$ in $\mathcal{P}^{\prime}$.)

G.5. We have quite generally for all $\lambda, \mu \in C_{0}$ and all simple $L$ in $\mathcal{C}_{\lambda}$

$$
T_{\lambda}^{\mu} Q_{L} \simeq \bigoplus_{L^{\prime}}\left(Q_{L^{\prime}}\right)^{m\left(L, L^{\prime}\right)}
$$

where $L^{\prime}$ runs over representatives of simple modules in $\mathcal{C}_{\mu}$ and

$$
m\left(L, L^{\prime}\right)=\operatorname{dim} \operatorname{Hom}\left(T_{\lambda}^{\mu} Q_{L}, L^{\prime}\right)=\operatorname{dim} \operatorname{Hom}\left(Q_{L}, T_{\mu}^{\lambda} L^{\prime}\right)=\left[T_{\mu}^{\lambda} L^{\prime}: L\right] .
$$

Lemma. Let $\lambda \in C_{0}^{\prime}$, let $\alpha$ be a short simple root with $\alpha \in J(\lambda)$. Then we have

$$
n(\alpha) Q_{\alpha}^{\lambda} \in \mathcal{P}_{\lambda}^{\prime} .
$$

Proof. Set $\mu=\varpi_{\alpha}-\rho$. We claim that

$$
Q_{\alpha}^{\lambda} \simeq T_{\mu}^{\lambda} Q_{\alpha}^{\mu}
$$

Then the claim follows from G.3(2) and Lemma G.4. In order to get (4), we use (1) and (2): If $L^{\prime}$ is a simple module in $\mathcal{C}_{\lambda}$ with $T_{\lambda}^{\mu} L^{\prime} \neq 0$, then $\alpha \in \kappa\left(L^{\prime}\right)$, hence $L^{\prime} \simeq L_{\alpha}^{\lambda}$ or $L^{\prime} \simeq L_{0}^{\lambda}$. It remains to recall that $T_{\lambda}^{\mu} L_{\alpha}^{\lambda} \simeq L_{\alpha}^{\mu}$ and $T_{\lambda}^{\mu} L_{0}^{\lambda} \simeq L_{0}^{\mu}$ by G.1.

Remark. Let $\lambda \in C_{0}^{\prime}$, let $\alpha$ be a long simple root with $\alpha \in J(\lambda)$. One gets similarly, using Remark G.4 instead of Lemma G.4 that $n(\alpha) Q_{\alpha}^{\lambda} \in \mathcal{P}_{\lambda}^{\prime}$ or $n(\alpha)\left(Q_{\alpha, 1}^{\lambda}+Q_{\alpha, 2}^{\lambda}\right) \in$ $\mathcal{P}_{\lambda}^{\prime}$. 
G.6. Recall the integers $m_{\beta}$ from D.6(3).

Theorem. Let $\lambda \in C_{0}^{\prime}$. Suppose that all roots in $R$ have the same length. Then we have

$$
\left[Q_{0}^{\lambda}: L_{0}^{\lambda}\right]=|W \cdot \lambda|
$$

and

$$
\left[Q_{\alpha}^{\lambda}: L_{0}^{\lambda}\right]=\left[Q_{0}^{\lambda}: L_{\alpha}^{\lambda}\right]=|W \cdot \lambda| m_{\alpha}
$$

for all $\alpha \in J(\lambda)$ and

$$
\left[Q_{\alpha}^{\lambda}: L_{\beta}^{\lambda}\right]=|W \cdot \lambda| m_{\alpha} m_{\beta}
$$

for all $\alpha, \beta \in J(\lambda)$.

Proof. We may assume that $\chi\left(\mathfrak{b}^{+}\right)=0$. Lemma G.5 and G.4(1) imply that we can apply G.3(5) to all simple modules $L$ and $L^{\prime}$ in $\mathcal{C}_{\lambda}$. We have

$$
|W \cdot(\lambda+p X)|=|W \cdot \lambda|
$$

by C.1. So the claim follows from $\left[Z_{\chi}(\lambda): L_{\alpha}^{\lambda}\right]=m_{\alpha}$ (see F.5) and $\left[Z_{\chi}(\lambda): L_{0}^{\lambda}\right]=1$ (see C.2).

Remarks. 1) This result confirms for $p$-regular $\lambda$ (i.e., for $\lambda \in C_{0}^{\prime}$ with $|W \cdot \lambda|=W$ ) the revised conjecture by Lusztig, as in [L4], 2.6. (The formulation there looks somewhat different, but can be checked to yield the same numbers.)

2) Suppose that $R$ is of type $B_{n}, C_{n}$, or $F_{4}$. Then we can apply G.3(5) for $L=L_{0}^{\lambda}$ and for $L=L_{\alpha}^{\lambda}$ with $\alpha$ short. Therefore (1) holds, (2) holds if $\alpha$ is short, and (3) holds if both $\alpha$ and $\beta$ are short. If $\beta$ is a long root in $J(\lambda)$ and if there (as expected) exist two isomorphism classes of simple modules with $\kappa$ invariant $\{\beta\}$ in $\mathcal{C}_{\lambda}$, then we get

$$
\left[Q_{0}^{\lambda}: L_{\beta, i}^{\lambda}\right]=|W \cdot \lambda| \frac{m_{\beta}}{2}
$$

and

$$
\left[Q_{\alpha}^{\lambda}: L_{\beta, i}^{\lambda}\right]=|W \cdot \lambda| \frac{m_{\alpha} m_{\beta}}{2}
$$

for all short $\alpha \in J(\lambda)$ [and for $i=1,2$ ] in both cases. By the symmetry of the Cartan matrix we get also $\left[Q_{\beta, i}^{\lambda}: L_{0}^{\lambda}\right]$ and $\left[Q_{\beta, i}^{\lambda}: L_{\alpha}^{\lambda}\right]$.

3) For $R$ of type $B_{n}$ we can choose $\chi$ to have standard Levi form. Then $Q_{L}$ has a $Z$-filtration for each simple $L$ in $\mathcal{C}$; cf. [J3], 10.11. This implies that G.3(5) holds for all $L$ even though $Q_{L} \notin \mathcal{P}^{\prime}$ in general (see the final remark in G.4). We get in this case that

$$
\left[Q_{\alpha, i}^{\lambda}: L_{\beta, j}^{\lambda}\right]=|W \cdot \lambda| \frac{m_{\alpha} m_{\beta}}{4}
$$

for all long $\alpha, \beta \in J(\lambda)$. One may speculate whether (8) also holds in types $C_{n}$ and $F_{4}$. 
H.

We keep the assumptions from Section D.

H.1. Recall from D.6(1) that we write $\alpha_{0}^{\vee}=\sum_{\beta \in \Pi} m_{\beta} \beta^{\vee}$ where $\Pi$ is the set of simple roots.

The fundamental weight $\varpi_{\alpha}$ corresponding to a simple root $\alpha$ is minuscule (in the sense of [Bou], Ch. VI, $\S 1$, exerc. 24) if and only if $m_{\alpha}=1$. For all $\alpha$ with this property set $y_{\alpha}=y_{\alpha}^{\prime} w_{0} \in W$ where $w_{0}$ is the longest element in $W$ and where $y_{\alpha}^{\prime}$ is the longest element in the subgroup of $W$ generated by all $s_{\beta}$ with $\beta \neq \alpha$.

Now Prop. 6 in [Bou], Ch. VI, §2, (applied to $R^{\vee}$ instead of $R$ ) shows that $y_{\alpha} \cdot C_{0}+p \varpi_{\alpha}=C_{0}$. More precisely, the map $x \mapsto y_{\alpha} \bullet x+p \varpi_{\alpha}$ maps the 'real' alcove of all $x \in X \otimes_{\mathbf{z}} \mathbf{R}$ with $0 \leq\left\langle x+\rho, \beta^{\vee}\right\rangle \leq p$ for all $\beta \in R^{+}$to itself. It therefore permutes the "walls" of this alcove, i.e., the hyperplanes with equations $\left\langle x+\rho, \beta^{\vee}\right\rangle=0$ with $\beta \in \Pi$ and the hyperplane with equation $\left\langle x+\rho, \alpha_{0}^{\vee}\right\rangle=p$. So $y_{\alpha}$ permutes $\Pi \cup\left\{-\alpha_{0}\right\}$. In fact, one checks easily that $y_{\alpha}\left(-\alpha_{0}\right)=\alpha$ and that

$$
\left\langle x+\rho, \alpha_{0}^{\vee}\right\rangle=p \Longleftrightarrow\left\langle y_{\alpha}(x+\rho)+p \varpi_{\alpha}, \alpha^{\vee}\right\rangle=0 .
$$

The simple root $-w_{0} \alpha$ satisfies $y_{\alpha}\left(-w_{0} \alpha\right)=-y_{\alpha}^{\prime}(\alpha)<0$ since $y_{\alpha}^{\prime}(\alpha)>0$. It follows that $y_{\alpha}\left(-w_{0} \alpha\right)=-\alpha_{0}$, hence that

$$
\left\langle x+\rho,\left(-w_{0} \alpha\right)^{\vee}\right\rangle=0 \Longleftrightarrow\left\langle y_{\alpha}(x+\rho)+p \varpi_{\alpha}, \alpha_{0}^{\vee}\right\rangle=p .
$$

If we apply $y_{\alpha}$ to $\alpha_{0}^{\vee}$, then we get easily that

$$
m_{-w_{0} \alpha}=1 \quad \text { and } \quad m_{y_{\alpha} \beta}=m_{\beta} \text { for all } \beta \in \Pi, \beta \neq-w_{0} \alpha .
$$

Proposition. Suppose that $R$ is not of exceptional type. Then there exists for each $\mu \in X$ a weight $\lambda \in C_{0}^{\prime}$ with $\mu \in W \bullet \lambda+p X$.

Proof. Since $C_{0}$ is a fundamental domain for $W_{p}$, we may assume that $\mu \in C_{0}$. If $\left\langle\mu+\rho, \alpha_{0}^{\vee}\right\rangle<p$, then $\mu \in C_{0}^{\prime}$ and we can take $\lambda=\mu$. So let us assume that $\left\langle\mu+\rho, \alpha_{0}^{\vee}\right\rangle=p$.

If there exists a simple root $\alpha$ with $\varpi_{\alpha}$ minuscule and $y_{\alpha} \bullet \mu+p \varpi_{\alpha} \in C_{0}^{\prime}$, then we take $\lambda=y_{\alpha} \bullet \mu+p \varpi_{\alpha}$. If not, then (2) shows that $\left\langle\mu+\rho,-w_{0} \alpha^{\vee}\right\rangle=0$ for all these $\alpha$. Since $-w_{0}$ is an involution, we get also $\left\langle\mu+\rho, \alpha^{\vee}\right\rangle=0$ for all simple $\alpha$ with $m_{\alpha}=1$. The assumption $\left\langle\mu+\rho, \alpha_{0}^{\vee}\right\rangle=p$ yields therefore

$$
p=\sum_{m_{\beta}>1} m_{\beta}\left\langle\mu+\rho, \beta^{\vee}\right\rangle \text {. }
$$

So far we did not use any assumption on $R$. We do that now. If $R$ is of type $A_{n}$, then we have $m_{\beta}=1$ for all $\beta \in \Pi$; so the right hand side in (4) is equal to 0 : a contradiction. If $R$ is of type $B_{n}$ or $C_{n}$ (with $n \geq 2$ ), or $D_{n}$ (with $n \geq 4$ ), then $m_{\beta} \in\{1,2\}$ for all $\beta \in \Pi$. Then (4) turns into

$$
p=2 \sum_{m_{\beta}=2}\left\langle\mu+\rho, \beta^{\vee}\right\rangle .
$$

Since $p \neq 2$ in these cases by (B2), we get a contradiction.

Remarks. 1) If we drop our assumptions (B1)-(D2), then we can extend the proposition to all cases where $R$ has no components of exceptional type provided that $p \neq 2$ if $R$ has a component not of type $A$.

The proposition above gives the result in case $G$ is semi-simple and $R$ indecomposable. If $G$ is semi-simple and $R$ arbitrary, then $X$ is the direct sum of the weight 
lattices of the irreducible components of $R$. These components are stable under $W$; we get the result for $X$ immediately from that for each component. For arbitrary reductive $G$ set $X_{0}$ equal to the subgroup of all $\nu \in X$ with $\left\langle\nu, \alpha^{\vee}\right\rangle=0$ for all $\alpha \in R$. Then $X / X_{0}$ identifies with the weight lattice of $R$, and the canonical map $X \rightarrow X / X_{0}$ commutes with the action of $W$. Therefore the claim for $X / X_{0}$ implies the claim for $X$.

2) It is clear that the proposition cannot extend to the types $E_{8}, F_{4}$, and $G_{2}$. In these cases $W \bullet \mu+p X=W_{p} \bullet \mu$; since $C_{0}$ is a fundamental domain for $W_{p}$, we cannot move any $\mu \in C_{0}$ with $\left\langle\mu+\rho, \alpha_{0}^{\vee}\right\rangle=p$ to an element in $C_{0}^{\prime}$.

For $R$ of type $E_{6}$ and $E_{7}$ the proof of the proposition shows that we can handle all $\mu \in C_{0}$ with $\left\langle\mu+\rho, \alpha_{0}^{\vee}\right\rangle=p$ for which there exists a simple root $\alpha$ with $m_{\alpha}=1$ and $\left\langle\mu+\rho, \alpha^{\vee}\right\rangle>0$. The simple roots with this property are $\alpha_{1}$ and $\alpha_{6}$ for $E_{6}$, resp. $\alpha_{7}$ for $E_{7}$ (in the numbering from [Bou]). However there will now exist other weights that we cannot handle since there exist simple roots $\beta, \gamma$ with $m_{\beta}=2$ and $m_{\gamma}=3$.

H.2. Let $\chi \in \mathfrak{g}^{*}$ be subregular nilpotent. Let $\lambda \in C_{0}$ be $p$-regular, i.e., with $0<\left\langle\lambda+\rho, \alpha^{\vee}\right\rangle<p$ for all positive roots $\alpha$. Consider a simple root $\beta$ such that $\varpi_{\beta}$ is minuscule. Then $\lambda^{\prime}=y_{\beta} \bullet \lambda+p \varpi_{\beta}$ belongs to $C_{0}$ (see H.1) and one checks easily that also $\lambda^{\prime}$ is $p$-regular. The translation functor $T_{\lambda}^{\lambda^{\prime}}$ is a selfequivalence of the category $\mathcal{C}_{\lambda}=\mathcal{C}_{\lambda^{\prime}}$.

Lemma. We have

$$
T_{\lambda}^{\lambda^{\prime}} L_{0}^{\lambda} \simeq L_{-y_{\beta}^{-1} \alpha_{0}}^{\lambda}
$$

and for all short simple roots $\gamma$

$$
T_{\lambda}^{\lambda^{\prime}} L_{\gamma}^{\lambda} \simeq \begin{cases}L_{y_{\beta}^{-1} \gamma}^{\lambda}, & \text { if } y_{\beta}^{-1} \gamma \in \Pi, \\ L_{0}^{\lambda}, & \text { if } y_{\beta}^{-1} \gamma=-\alpha_{0} .\end{cases}
$$

Proof. It is enough to prove this for one choice of $\chi$. So we may assume that there exists a short simple root $\alpha$ with $\chi\left(\mathfrak{p}_{\alpha}\right)=0$. Consider (2). Choose $w \in W$ with $w^{-1} \alpha=\gamma$. Then $L_{\gamma}^{\lambda}$ is isomorphic to $Z_{\chi}(w \bullet \lambda, \alpha)$, since the latter module is simple and has the right $\kappa$ invariant; see D.3.a, D.4.a, D.5.c. It follows that

$$
T_{\lambda}^{\lambda^{\prime}} L_{\gamma}^{\lambda} \simeq Z_{\chi}\left(w \cdot \lambda^{\prime}, \alpha\right)=Z_{\chi}\left(w y_{\beta} \cdot \lambda, \alpha\right) .
$$

Now (2) follows using again D.3.a, D.4.a, D.5.c, since $\left(w y_{\beta}\right)^{-1} \alpha=y_{\beta}^{-1} \gamma$.

The proof of (1) is analogous; now one has to choose $w \in W$ with $w^{-1} \alpha=-\alpha_{0}$.

Remarks. 1) One can show similarly: If $\gamma$ is a long simple root, then $T_{\lambda}^{\lambda^{\prime}}$ takes a simple module with $\kappa$ invariant $\gamma$ to one with $\kappa$ invariant $y_{\beta}^{-1} \gamma$.

If $R$ is of type $B_{n}$ or $C_{n}$, then there exists exactly one simple $\operatorname{root} \beta$ with $\varpi_{\beta}$ minuscule: We have $\beta=\alpha_{n}$ in the $B_{n}$ case, and $\beta=\alpha_{1}$ in the $C_{n}$ case.

For $R$ of type $C_{n}$ one then easily checks that $y_{\beta}=s_{2 \varepsilon_{1}}$. The only simple modules not covered by the lemma are those with $\kappa$ invariant $\left\{\alpha_{n}\right\}$. If we choose $\chi$ as in F.6(2), then $L=Z_{\chi}\left(\lambda, \alpha_{n}\right)$ is one of these simple modules. We get then

$$
T_{\lambda}^{\lambda^{\prime}} L \simeq Z_{\chi}\left(s_{2 \varepsilon_{1}} \bullet \lambda, \alpha_{n}\right) \simeq{ }^{g} L
$$

with $g$ as in F.10. So we see that $T_{\lambda}^{\lambda^{\prime}}$ interchanges, in this case, the two (expected) simple modules corresponding to $\alpha_{n}$. 
In type $B_{n}$ one checks similarly that $T_{\lambda}^{\lambda^{\prime}} L_{\chi}\left(\lambda_{i}\right) \simeq L_{\chi}\left(\lambda_{n+i}\right)$ for $1 \leq i<n$ in the notations from [J2].

2) Note that Lemma G.1 says $T_{\lambda}^{\lambda^{\prime}} L_{\gamma}^{\lambda} \simeq L_{\gamma}^{\lambda^{\prime}}$ for all $\gamma$ as in (2). This illustrates that $\kappa$ depends (as mentioned in D.5) on the choice of $\lambda$, not just on the category $\mathcal{C}_{\lambda}$.

H.3. So far we have studied only the categories $\mathcal{C}_{\lambda}$ with $\lambda \in C_{0}^{\prime}$. In order to get all possible $\mathcal{C}_{\lambda}$ we should also look at the $\lambda \in C_{0}$ with $\left\langle\lambda+\rho, \alpha_{0}^{\vee}\right\rangle=p$. We know from H.1 that there exist in many cases $\lambda^{\prime} \in C_{0}^{\prime}$ with $\mathcal{C}_{\lambda}=\mathcal{C}_{\lambda^{\prime}}$, but there are also cases where this does not hold.

When we try to extend the theory for weights in $C_{0}^{\prime}$ to the remaining weights in $C_{0}$, then we encounter two major problems: The proof in D.3 that certain modules are simple will not work for all $\lambda \in C_{0}$, and there is not an easy definition of a $\kappa$ invariant as in D.5. The first problem occurs only for relatively few $\lambda$ and we are going to ignore them here.

Definition. We say that a weight $\lambda \in C_{0}$ is nice if for all simple roots $\alpha$, all subregular $\chi \in \mathfrak{g}^{*}$ with $\chi\left(\mathfrak{p}_{\alpha}\right)=0$, and all $w \in W$ with $w^{-1} \alpha$ simple and $\left\langle w(\lambda+\rho), \alpha^{\vee}\right\rangle>0$ the module $Z_{\chi}(w \bullet \lambda, \alpha)$ is simple.

Note that Lemma D.3.a says that each $\lambda \in C_{0}^{\prime}$ is nice. I hope that all $\lambda \in C_{0}$ are nice in good characteristic; what I can prove is this:

Lemma. Let $\lambda \in C_{0}$ with $\left\langle\lambda+\rho, \alpha_{0}^{\vee}\right\rangle=p$.

a) In case $R$ is of type $E_{8}, F_{4}$, or $G_{2}$, assume that $p>h+1$. If there exists a simple root $\beta$ with $\varpi_{\beta}$ minuscule such that $y_{\beta} \bullet \lambda+p \varpi_{\beta} \in C_{0}^{\prime}$, then $\lambda$ is nice.

b) If there exists for each simple root $\gamma$ with $\left\langle\lambda+\rho, \gamma^{\vee}\right\rangle>0$ a weight $\mu$ in the same facet as $\lambda$ with $\left\langle\mu+\rho, \gamma^{\vee}\right\rangle=1$, then $\lambda$ is nice.

c) Suppose that $R$ is not of type $G_{2}$. If $\left\langle\lambda+\rho, \gamma^{\vee}\right\rangle>0$ for all simple roots $\gamma$, then $\lambda$ is nice.

Proof. Consider $\alpha, \chi$, and $w$ as in the definition above.

a) Set $\lambda^{\prime}=y_{\beta} \bullet \lambda+p \varpi_{\beta}$. We have

$$
Z_{\chi}(w \cdot \lambda, \alpha) \simeq Z_{\chi}\left(w y_{\beta}^{-1} \cdot \lambda^{\prime}, \alpha\right) .
$$

Recall from H.1 that $y_{\beta}$ permutes $\Pi \cup\left\{-\alpha_{0}\right\}$. We have therefore

$$
\left(w y_{\beta}^{-1}\right)^{-1} \alpha=y_{\beta} w^{-1} \alpha \in \Pi \cup\left\{-\alpha_{0}\right\} .
$$

So the right hand side in (1) is simple by Lemma D.4.a (in case $y_{\beta} w^{-1} \alpha=-\alpha_{0}$ ) or by Lemma D.3.a (in case $y_{\beta} w^{-1} \alpha$ simple) provided we know in the second case that $\left\langle w y_{\beta}^{-1}\left(\lambda^{\prime}+\rho\right), \alpha^{\vee}\right\rangle>0$. However, one checks easily that

$$
\left\langle w y_{\beta}^{-1}\left(\lambda^{\prime}+\rho\right), \alpha^{\vee}\right\rangle=\left\langle w(\lambda+\rho), \alpha^{\vee}\right\rangle+p\left\langle\varpi_{\beta}, y_{\beta} w^{-1} \alpha^{\vee}\right\rangle>0
$$

using $w y_{\beta}^{-1}\left(\lambda^{\prime}+\rho\right)=w(\lambda+\rho)+p w y_{\beta}^{-1} \varpi_{\beta}$.

b) Under these assumptions Lemma D.2 holds with $F(J)$ replaced by the facet of $\lambda$. Therefore the proof of Lemma D.3.a works in this case.

c) Note that $p=\left\langle\lambda+\rho, \alpha_{0}^{\vee}\right\rangle$ and $\lambda \in C_{0}$ imply $p \geq\left\langle\rho, \alpha_{0}^{\vee}\right\rangle=h-1$.

If $R$ is of type $A_{1}$, then $\chi=0$ and $w=1$. Then $Z_{\chi}(\lambda, \alpha)=Z_{\chi}(\lambda)$ is a Steinberg module, hence irreducible.

Assume that $R$ is not of type $A_{1}$. I want to show that the assumption in $\mathrm{b}$ ) is satisfied. Let $\gamma$ be a simple root. If $\varpi_{\beta}$ is a minuscule fundamental weight with $\beta \neq \gamma$, then we can choose $\mu=\left(p-\left\langle\rho, \alpha_{0}^{\vee}\right\rangle\right) \varpi_{\beta}$. 
We are left with the cases where there are no minuscule fundamental weights at all, or where $\varpi_{\gamma}$ is the only minuscule fundamental weight. (So we are not in type $A_{n}$.) In these cases one can find a fundamental weight $\varpi_{\beta}$ with $\left\langle\varpi_{\beta}, \alpha_{0}^{\vee}\right\rangle=2$ and $\beta \neq \gamma$. (Recall that we exclude $G_{2}$.) Furthermore both $\left\langle\rho, \alpha_{0}^{\vee}\right\rangle$ and $p$ are odd (since we are done with type $\left.A_{n}\right)$. So we can choose $\mu=r \varpi_{\beta}$ with $r=\left(p-\left\langle\rho, \alpha_{0}^{\vee}\right\rangle\right) / 2$.

Remark. If $R$ is of exceptional type, then there exist simple roots $\beta$ and $\gamma$ with $\left\langle\varpi_{\beta}, \alpha_{0}^{\vee}\right\rangle=2$ and $\left\langle\varpi_{\gamma}, \alpha_{0}^{\vee}\right\rangle=3$. Consider positive integers $a$ and $b$ with $2 a+3 b=p$. (They exist for each $p \geq 5$.) Then $\lambda=a \varpi_{\beta}+b \varpi_{\gamma}-\rho$ satisfies $\left\langle\lambda+\rho, \alpha_{0}^{\vee}\right\rangle=p$. The weights in the facet of $\lambda$ are all $\mu=a^{\prime} \varpi_{\beta}+b^{\prime} \varpi_{\gamma}-\rho$ with $a^{\prime}, b^{\prime}>0$ and $2 a^{\prime}+3 b^{\prime}=p$. We have $\left\langle\mu+\rho, \beta^{\vee}\right\rangle=1$ if and only if $a^{\prime}=1$, if and only if $3 b^{\prime}=p-2$. So we can find $\mu$ with this property only in case $p \equiv 2(\bmod 3)$. So $\lambda$ does not satisfy the condition in b). Using Remark 2 in H.1 one can see that $\lambda$ also does not satisfy the condition in a).

H.4. Let $\mu \in C_{0}$ with $\left\langle\mu+\rho, \alpha_{0}^{\vee}\right\rangle=p$. Suppose that $\lambda$ is a weight with $\lambda \in C_{0}^{\prime}$ such that $\mu$ is in the closure of the facet of $\lambda$. (Given $\mu$ we can find $\lambda$ with this property as long as there exists a simple root $\alpha$ with $\left\langle\mu+\rho, \alpha^{\vee}\right\rangle>1$ : In that case $\lambda=\mu-\varpi_{\alpha}$ works. If there is no such $\alpha$, then $\mu+\rho=\sum_{\alpha \in J(\mu)} \varpi_{\alpha}$ and then no $\lambda$ as above can exist. This can happen only in case $p \leq h-1$.)

Lemma. Let $\mu$ and $\lambda$ be as above. Let $\alpha$ be a simple root and let $\chi \in \mathfrak{g}^{*}$ with $\chi\left(\mathfrak{p}_{\alpha}\right)=0$. Let $w \in W$.

a) We have $T_{\lambda}^{\mu} Z_{\chi}(w \cdot \lambda, \alpha)=0$ if $w^{-1} \alpha<0$ and $\left\langle w(\mu+\rho), \alpha^{\vee}\right\rangle=-p$ or if $w^{-1} \alpha>0$ and $\left\langle w(\mu+\rho), \alpha^{\vee}\right\rangle=0<\left\langle w(\lambda+\rho), \alpha^{\vee}\right\rangle$. In all other cases we have $T_{\lambda}^{\mu} Z_{\chi}(w \bullet \lambda, \alpha) \simeq Z_{\chi}(w \bullet \mu, \alpha)$.

b) Suppose that $\left\langle w(\mu+\rho), \alpha^{\vee}\right\rangle \not \equiv 0(\bmod p)$. Then $T_{\mu}^{\lambda} Z_{\chi}(w \bullet \mu, \alpha)$ has a filtration with factors $Z_{\chi}\left(w \cdot \lambda^{\prime}, \alpha\right)$ with $\lambda^{\prime} \in\left(\operatorname{Stab}_{W_{p}} \mu\right) \cdot \lambda$.

Proof. a) This is more or less a special case of Corollary B.11. One uses that $\left\langle w(\lambda+\rho), \alpha^{\vee}\right\rangle>-p$ for all $w \in W$.

b) We apply Proposition B.7 with $I=\{\alpha\}$ to $\left(w_{\bullet} \lambda, w \bullet \mu\right)$ instead of $(\lambda, \mu)$. Since $w \bullet \mu$ is in the closure of the facet of $w_{\bullet} \lambda$, the assumption $\left\langle w(\mu+\rho), \alpha^{\vee}\right\rangle \not \equiv 0(\bmod p)$ implies that also $\left\langle w(\lambda+\rho), \alpha^{\vee}\right\rangle \not \equiv 0(\bmod p)$. So both $w_{\bullet} \mu$ and $w_{\bullet} \lambda$ have trivial stabiliser in $W_{I, p}$. Proposition B.7 says now that

$$
T_{\mu}^{\lambda} Z_{\chi}(w \bullet \mu, \alpha)=T_{w \bullet \mu}^{w \bullet \lambda} \operatorname{ind}_{I} L_{\chi, \alpha}(w \bullet \mu)
$$

has a filtration with factors

$$
\operatorname{ind}_{I} T(I)_{w \bullet \mu}^{w_{i} w_{\bullet} \lambda} L_{\chi, \alpha}(w \bullet \mu)
$$

where the $w_{i}$ belong to $\operatorname{Stab}_{W_{p}} w_{\bullet} \mu$ and are a system of representatives for the cosets modulo $\operatorname{Stab}_{W_{p}} w_{\bullet} \lambda$.

Now $w_{\bullet} \mu$ and each $w_{i} w_{\bullet} \lambda$ belong to the same (open) alcove with respect to $W_{I, p}$. This implies that $T(I)_{w \bullet \mu}^{w_{i} w_{\bullet} \lambda} L_{\chi, \alpha}\left(w_{\bullet} \mu\right) \simeq L_{\chi, \alpha}\left(w_{i} w_{\bullet} \lambda\right)$. So the module in (1) is isomorphic to $Z_{\chi}\left(w_{i} w_{\bullet} \lambda, \alpha\right)$. The claim follows since the $x w_{\bullet} \lambda$ with $x \in \operatorname{Stab}_{W_{p}} w_{\bullet} \mu$ are precisely the $w y \bullet \lambda$ with $y \in \operatorname{Stab}_{W_{p}} \mu$.

H.5. Suppose that $\mu$ and $\lambda$ are weights as in H.4. We return to the assumptions and notations from G.1. In particular, we suppose that $\chi$ is subregular nilpotent.

Lemma. Assume that $\mu$ is nice. 
a) If $L$ is a simple module in $\mathcal{C}_{\lambda}$, then $T_{\lambda}^{\mu} L$ is either 0 or simple. We have $T_{\lambda}^{\mu} L=0$ if and only if $L \simeq L_{0}^{\lambda}$ or $\kappa(L)=\{\gamma\}$ with $\gamma \notin J(\mu)$.

b) Each simple module $L^{\prime}$ in $\mathcal{C}_{\mu}$ is isomorphic to some non-zero $T_{\lambda}^{\mu} L$ as in a). We have

$$
\left[T_{\lambda}^{\mu} T_{\mu}^{\lambda} L^{\prime}\right]=\left(\operatorname{Stab}_{W_{p}}(\mu): \operatorname{Stab}_{W_{p}}(\lambda)\right)\left[L^{\prime}\right]
$$

in the Grothendieck group of $\mathcal{C}_{\mu}$.

Proof. a) Suppose first that $L \simeq L_{0}^{\lambda}$ or $\kappa(L)=\{\gamma\}$ with $\gamma$ short. Using B.13(2) and D.1 one reduces to the case where $\chi\left(\mathfrak{p}_{\alpha}\right)=0$ for some short simple root $\alpha$. We have then $w \in W$ with $L \simeq Z_{\chi}(w \bullet \lambda, \alpha)$ and $w^{-1} \alpha=-\alpha_{0}$ or $w^{-1} \alpha=\gamma$. Now Lemma H.4 yields $T_{\lambda}^{\mu} L=0$ in case $w^{-1} \alpha=-\alpha_{0}$ or $w^{-1} \alpha=\gamma$ with $\gamma \notin J(\mu)$. In the remaining cases the translated module is isomorphic to $Z_{\chi}(w \bullet \mu, \alpha)$, hence simple by the definition of 'nice'.

In case $\kappa(L)=\{\gamma\}$ with $\gamma$ long, one argues similarly with $\alpha$ long.

b) We may assume that $\chi\left(\mathfrak{b}^{+}\right)=0$. Each simple module $L^{\prime}$ in $\mathcal{C}_{\mu}$ is a composition factor of $Z_{\chi}(\mu)$. Since $Z_{\chi}(\mu) \simeq T_{\lambda}^{\mu} Z_{\chi}(\lambda)$, the exactness of $T_{\lambda}^{\mu}$ implies that $L^{\prime}$ is a composition factor of some $T_{\lambda}^{\mu} L$ with $L$ a composition factor of $Z_{\chi}(\lambda)$. Using a) this yields the first claim in b).

Suppose that $L^{\prime}=T_{\lambda}^{\mu} L$ with $L$ and $L^{\prime}$ as above. There exists a simple root $\beta \in J(\mu) \subset J(\lambda)$ such that $\kappa(L)=\{\gamma\}$. We may assume that there exists a simple root $\alpha$ of the same length as $\gamma$ such that $\chi\left(\mathfrak{p}_{\alpha}\right)=0$. Then there exists $w \in W$ with $w \gamma=\alpha$ such that $L \simeq Z_{\chi}(w \bullet \lambda, \alpha)$ and $L^{\prime} \simeq Z_{\chi}(w \bullet \mu, \alpha)$.

Suppose first that $\left\langle w(\mu+\rho), \alpha^{\vee}\right\rangle<p$. Then Lemma H.4.b implies that $T_{\mu}^{\lambda} L^{\prime}$ has a filtration with factors $Z_{\chi}\left(w w^{\prime} \bullet \lambda, \alpha\right)$ with $w^{\prime}$ running over representatives for the cosets of $\operatorname{Stab}_{W_{p}}(\lambda)$ in $\operatorname{Stab}_{W_{p}}(\mu)$. Each of these factors satisfies

$$
T_{\lambda}^{\mu} Z_{\chi}\left(w w^{\prime} \bullet \lambda, \alpha\right) \simeq Z_{\chi}\left(w w^{\prime} \bullet \mu, \alpha\right)=Z_{\chi}(w \bullet \mu, \alpha) \simeq L .
$$

This yields the claim in this case.

Finally, if $p=\left\langle w(\mu+\rho), \alpha^{\vee}\right\rangle=\left\langle\mu+\rho, \gamma^{\vee}\right\rangle$, then necessarily $\mu+\rho=p \varpi_{\gamma}$ and $\varpi_{\gamma}$ minuscule. In this case one gets $L^{\prime} \simeq Z_{\chi}(w \bullet \mu)$ and one can argue similarly using Proposition B.3.

Remark. If $L^{\prime}$ is a simple module in $\mathcal{C}_{\mu}$, then a) and G.5(1),(2) imply

$$
T_{\mu}^{\lambda} Q_{L^{\prime}} \simeq \bigoplus_{T_{\lambda}^{\mu} L \simeq L^{\prime}} Q_{L}
$$

(Here $L$ runs over representatives of isomorphism classes.) If all $Q_{L}$ occurring in (2) satisfy G.3(3), i.e.,

$$
\text { if } \quad\left[Q_{L}\right]=\frac{\operatorname{dim} Q_{L}}{p^{N}}\left[Z_{\chi}(\lambda)\right] \quad \text { for all } L \text { with } T_{\lambda}^{\mu} L \simeq L^{\prime},
$$

then the exactness of $T_{\lambda}^{\mu}$ yields

$$
\left[T_{\lambda}^{\mu} Q_{L}\right]=\frac{\operatorname{dim} Q_{L}}{p^{N}}\left[Z_{\chi}(\mu)\right]=\frac{\operatorname{dim} T_{\lambda}^{\mu} Q_{L}}{p^{N}}\left[Z_{\chi}(\mu)\right] .
$$

Combining (2) and (1) we get

$$
\left(\operatorname{Stab}_{W_{p}}(\mu): \operatorname{Stab}_{W_{p}}(\lambda)\right)\left[Q_{L^{\prime}}\right]=\left[T_{\lambda}^{\mu} T_{\mu}^{\lambda} Q_{L^{\prime}}\right]=\sum_{T_{\lambda}^{\mu} L \simeq L^{\prime}}\left[T_{\lambda}^{\mu} Q_{L}\right],
$$


hence

$$
\left[Q_{L^{\prime}}\right]=\frac{\operatorname{dim} Q_{L^{\prime}}}{p^{N}}\left[Z_{\chi}(\mu)\right]
$$

In other words, $Q_{L^{\prime}}$ satisfies G.3(3), if all $Q_{L}$ in (2) do. (We see also that all simple modules in $\mathcal{C}_{\mu}$ are composition factors of $Q_{L^{\prime}}$, hence that $\mathcal{C}_{\mu}$ is a block, as proved in general in $[\mathrm{BG}]$.)

H.6. Let $\alpha$ be a simple root, let $\chi \in \mathfrak{g}^{*}$ be subregular with $\chi\left(\mathfrak{p}_{\alpha}\right)=0$. We need some information on the composition factors of $Z_{\chi}(w \bullet \lambda, \alpha)$ with $\lambda \in C_{0}^{\prime}$ and $w \in W$. Define integers $m(w, \beta)$ for each simple $\operatorname{root} \beta$ such that

$$
\sum_{\beta} m(w, \beta) \beta^{\vee}= \begin{cases}w^{-1} \alpha^{\vee}, & \text { if } w^{-1} \alpha>0, \\ w^{-1} \alpha^{\vee}+\alpha_{0}^{\vee}, & \text { if } w^{-1} \alpha<0 .\end{cases}
$$

Lemma. Let $\lambda \in C_{0}^{\prime}$ and $w \in W$. We have

$$
\left[Z_{\chi}(w \bullet \lambda, \alpha): L_{0}^{\lambda}\right]= \begin{cases}0, & \text { if } w^{-1} \alpha>0 \\ 1, & \text { if } w^{-1} \alpha<0\end{cases}
$$

Let $\beta \in J(\lambda)$. If there is up to isomorphism only one simple module $L_{\beta}^{\lambda}$ in $\mathcal{C}_{\lambda}$ with $\kappa\left(L_{\beta}^{\lambda}\right)=\{\beta\}$, then

$$
\left[Z_{\chi}(w \bullet \lambda, \alpha): L_{\beta}^{\lambda}\right]=m(w, \beta)
$$

otherwise we get

$$
\left[Z_{\chi}(w \bullet \lambda, \alpha): L_{\beta, 1}^{\lambda}\right]+\left[Z_{\chi}(w \bullet \lambda, \alpha): L_{\beta, 2}^{\lambda}\right]=m(w, \beta) .
$$

Proof. We have $\left[Z_{\chi}\left(w_{\bullet} \lambda\right): L_{0}^{\lambda}\right]=1$ by Proposition C.2. So the multiplicity in (2) is either 0 or 1 . Corollary B.11 implies that $T_{\lambda}^{-\rho} Z_{\chi}(w \bullet \lambda, \alpha)=0$ if and only if $w^{-1} \alpha>0$. Now (2) follows from C.4.

Consider next (3). Using Lemma G.1 we see that

$$
T_{\lambda}^{\varpi_{\beta}-\rho} Z_{\chi}(w \cdot \lambda, \alpha) \simeq Z_{\chi}\left(w_{\bullet}\left(\varpi_{\beta}-\rho\right), \alpha\right)
$$

has $\left[Z_{\chi}(w \cdot \lambda, \alpha): L_{\beta}^{\lambda}\right]$ composition factors isomorphic to $T_{\lambda}^{\varpi_{\beta}-\rho} L_{\beta}^{\lambda} \simeq L_{\beta}^{\varpi_{\beta}-\rho}$; in case $w^{-1} \alpha>0$ there is an additional composition factor isomorphic to $T_{\lambda}^{\varpi_{\beta}-\rho} L_{0}^{\lambda} \simeq$ $L_{0}^{\varpi_{\beta}-\rho}$. We have

$$
\operatorname{dim} L_{\beta}^{\varpi_{\beta}-\rho}=p^{N-1}, \quad \operatorname{dim} L_{0}^{\varpi_{\beta}-\rho}=\left(p-\left\langle\varpi_{\beta}, \alpha_{0}^{\vee}\right\rangle\right) p^{N-1},
$$

and

$$
\operatorname{dim} Z_{\chi}\left(w_{\bullet}\left(\varpi_{\beta}-\rho\right), \alpha\right)= \begin{cases}\left\langle\varpi_{\beta}, w^{-1} \alpha^{\vee}\right\rangle p^{N-1}, & \text { if } w^{-1} \alpha>0, \\ \left(p+\left\langle\varpi_{\beta}, w^{-1} \alpha^{\vee}\right\rangle\right) p^{N-1}, & \text { if } w^{-1} \alpha<0 ;\end{cases}
$$

see A.4. Comparing dimensions we get

$$
\left[Z_{\chi}(w \bullet \lambda, \alpha): L_{\beta}^{\lambda}\right]= \begin{cases}\left\langle\varpi_{\beta}, w^{-1} \alpha^{\vee}\right\rangle, & \text { if } w^{-1} \alpha>0, \\ \left\langle\varpi_{\beta}, w^{-1} \alpha^{\vee}\right\rangle+\left\langle\varpi_{\beta}, \alpha_{0}^{\vee}\right\rangle, & \text { if } w^{-1} \alpha<0 .\end{cases}
$$

Plugging in (1), we get (3). The proof of (4) is analogous.

Remark. Note that (2) holds (by the same proof as above) for all $\chi \in \mathfrak{g}^{*}$ with $\chi\left(\mathfrak{p}_{\alpha}\right)=0$, not only for subregular $\chi$. 
H.7. Return to the situation from Lemma H.5. So we consider a weight $\lambda \in C_{0}^{\prime}$ and a weight $\mu$ in the closure of the facet of $\lambda$ with $\left\langle\mu+\rho, \alpha_{0}^{\vee}\right\rangle=p$ such that $\mu$ is nice.

Lemma. If $L_{1}$ and $L_{2}$ are simple modules in $\mathcal{C}_{\lambda}$ with $T_{\lambda}^{\mu} L_{1} \simeq T_{\lambda}^{\mu} L_{2} \neq 0$, then $\kappa\left(L_{1}\right)=\kappa\left(L_{2}\right)$.

Proof. Lemma H.5.a implies that there exists a simple root $\gamma \in J(\mu)$ with $\kappa\left(L_{1}\right)=$ $\{\gamma\}$. If $J(\mu)=\{\gamma\}$, then Lemma H.5.a implies that also $\kappa\left(L_{2}\right)=\{\gamma\}$, hence our claim. So assume from now on that $|J(\mu)|>1$.

We may assume that there exists a simple root $\alpha$ of the same length as $\gamma$ such that $\chi\left(\mathfrak{p}_{\alpha}\right)=0$. Then there exists $w \in W$ with $w \gamma=\alpha$ and $L_{1} \simeq Z_{\chi}(w \bullet \lambda, \alpha)$. Using

$$
\operatorname{Hom}_{\mathfrak{g}}\left(L_{2}, T_{\mu}^{\lambda} T_{\lambda}^{\mu} L_{1}\right) \simeq \operatorname{Hom}_{\mathfrak{g}}\left(T_{\lambda}^{\mu} L_{2}, T_{\lambda}^{\mu} L_{1}\right) \simeq K
$$

we see that $\left[T_{\mu}^{\lambda} T_{\lambda}^{\mu} L_{1}: L_{2}\right] \neq 0$. Lemma H.4 implies that $T_{\mu}^{\lambda} T_{\lambda}^{\mu} L_{1}$ has a filtration with factors of the form $Z_{\chi}\left(w w_{1} \bullet \lambda, \alpha\right)$ with $w_{1} \in W_{p}, w_{1} \bullet \mu=\mu$. (We need here $|J(\mu)|>1$ in order to get the assumption in H.4.b.)

It follows that $\left[Z_{\chi}\left(w w_{1} \bullet \lambda, \alpha\right): L_{2}\right] \neq 0$ for one of these $w_{1}$. We can write $w_{1}$ as a composition of some $w^{\prime} \in W$ with a translation by a weight in $p \mathbf{Z} R$ and get $Z_{\chi}\left(w w_{1} \bullet \lambda, \alpha\right) \simeq Z_{\chi}\left(w w^{\prime} \bullet \lambda, \alpha\right)$. We want to apply Lemma H.6 to this module. Note that $w^{\prime}$ is a product of reflections $s_{\beta}$ with $\beta$ in the union of $\left\{\alpha_{0}\right\}$ and of the set $\Pi^{\prime}$ of simple roots not in $J(\mu)$. So there are integers $c$ and $c_{\beta}$ with

$$
\left(w w^{\prime}\right)^{-1} \alpha^{\vee}=\gamma^{\vee}+c \alpha_{0}^{\vee}+\sum_{\beta \in \Pi^{\prime}} c_{\beta} \beta^{\vee} .
$$

Let $\gamma^{\prime} \in J(\mu)$ with $\gamma^{\prime} \neq \gamma$. If we write $\left(w w^{\prime}\right)^{-1} \alpha^{\vee}$ as a linear combination of the $\beta^{\vee}$ with $\beta$ simple, then $\gamma^{\vee v}$ occurs with coefficient $\mathrm{cm}_{\gamma^{\prime}}$ with $m_{\gamma^{\prime}}$ as in D.6(1). Since $\alpha_{0}^{\vee}$ is the largest root of $R^{\vee}$ this implies $\left|c m_{\gamma^{\prime}}\right| \leq m_{\gamma^{\prime}}$, hence $|c| \leq 1$. If $c=1$, then $\gamma^{\vee}$ occurs above with coefficient $1+m_{\gamma}$, a contradiction. So we get $c=0$ or $c=-1$. If $c=0$, then a look at the coefficient of $\gamma^{\vee}$ implies $\left(w w^{\prime}\right)^{-1} \alpha>0$; if $c=-1$, then a look at the coefficient of $\gamma^{\prime \vee}$ [with $\gamma^{\prime}$ as before] implies $\left(w w^{\prime}\right)^{-1} \alpha<0$. Now Lemma H.6 shows that $Z_{\chi}\left(w w^{\prime} \bullet \lambda, \alpha\right)$ has a composition factor $L_{1}^{\prime}$ with $\kappa$ invariant $\{\gamma\}$. All remaining composition factors have a $\kappa$ invariant in $\Pi^{\prime}$ or are isomorphic to $L_{0}^{\lambda}$, hence are killed by $T_{\lambda}^{\mu}$. On the other hand, $L_{2}$ is a composition factor of $Z_{\chi}\left(w w^{\prime} \bullet \lambda, \alpha\right)$ that is not killed by $T_{\lambda}^{\mu}$. This implies $L_{2} \simeq L_{1}^{\prime}$, hence the claim.

Remark. If all roots in $R$ have the same length, then Theorem F.5 implies now $L_{2} \simeq L_{1}$. Furthermore H.6(2) simplifies now to

$$
T_{\mu}^{\lambda} Q_{T_{\lambda}^{\mu} L} \simeq Q_{L}
$$

for all simple $L$ in $\mathcal{C}_{\lambda}$ with $T_{\lambda}^{\mu} L \neq 0$.

If $R$ is of type $B_{n}, C_{n}$, or $F_{4}$, then we get the same result as long as $\kappa\left(L_{1}\right)$ is a short simple root. If $\gamma$ is a long simple root in $J(\mu)$, then we cannot exclude that there are two non-isomorphic simple modules $L_{\gamma, 1}^{\lambda}$ and $L_{\gamma, 2}^{\lambda}$ that have the same image under translation. We shall look in $\mathrm{H} .11$ at a special case where this does not happen. I expect that it never occurs. 
H.8. For the remaining subsections fix a $p$-regular weight $\lambda$. The existence of $\lambda$

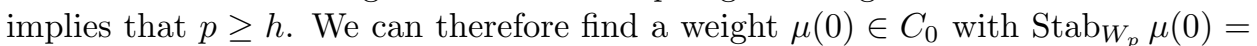
$\left\{1, s_{0}\right\}$ where $s_{0}=s_{\alpha_{0}, p}$ and we can find for each simple root $\beta$ a weight $\mu(\beta) \in C_{0}$ with $\operatorname{Stab}_{W_{p}} \mu(\beta)=\left\{1, s_{\beta}\right\}$; cf. [J1], II.6.3(1). So $J(\mu(0))$ is the set $\Pi$ of all simple roots whereas $J(\mu(\beta))=\Pi \backslash\{\beta\}$ otherwise.

We want to evaluate the "translations through the walls" functors

$$
\Theta_{\beta}=T_{\mu(\beta)}^{\lambda} \circ T_{\lambda}^{\mu(\beta)}, \quad \beta \in \Pi \cup\{0\}
$$

on our simple modules. There is one trivial case: We have for all $\beta$

$$
\Theta_{\beta} L_{\beta}^{\lambda}=0 \quad \text { resp. } \quad \Theta_{\beta} L_{\beta, i}^{\lambda}=0,
$$

since already $T_{\lambda}^{\mu(\beta)}$ annihilates these modules; see G.1 or H.5.

Lemma. a) Let $\beta, \gamma \in \Pi$ with $\beta \neq \gamma$. If $L$ is a simple module in $\mathcal{C}_{\lambda}$ with $\kappa(L)=\{\gamma\}$, then $\Theta_{\beta} L$ has 2 composition factors with $\kappa$ invariant $\{\gamma\}$ and $-\left\langle\beta, \gamma^{\vee}\right\rangle$ composition factors with $\kappa$ invariant $\{\beta\}$.

b) Let $\gamma \in \Pi$. If $L$ is a simple module in $\mathcal{C}_{\lambda}$ with $\kappa(L)=\{\gamma\}$, then $\Theta_{0} L$ has 2 composition factors with $\kappa$ invariant $\{\gamma\}$ and $\left\langle\alpha_{0}, \gamma^{\vee}\right\rangle$ composition factors isomorphic to $L_{0}^{\lambda}$. On the other hand, $\Theta_{\gamma} L_{0}^{\lambda}$ has 2 composition factors isomorphic to $L_{0}^{\lambda}$ and $\left\langle\gamma, \alpha_{0}^{\vee}\right\rangle$ composition factors with $\kappa$ invariant $\{\gamma\}$.

Proof. When looking at $\Theta_{\beta} L$ or at $\Theta_{0} L$ with $\kappa(L)=\{\gamma\}$, then we may assume that there exists a simple root $\alpha$ and $w \in W$ with $w \gamma=\alpha$ such that $\chi\left(\mathfrak{p}_{\alpha}\right)=0$ and $L \simeq Z_{\chi}\left(w_{\bullet} \lambda, \alpha\right)$.

Arguing as in H.4 one checks that $\Theta_{\beta} L$ has a filtration with factors $Z_{\chi}(w \bullet \lambda, \alpha) \simeq L$ and $Z_{\chi}\left(w s_{\beta} \bullet \lambda, \alpha\right)$. We want to apply Lemma H.6 to the second factor. We have

$$
\left(w s_{\beta}\right)^{-1} \alpha^{\vee}=s_{\beta} \gamma^{\vee}=\gamma^{\vee}-\left\langle\beta, \gamma^{\vee}\right\rangle \beta^{\vee} .
$$

Now the claim in a) follows immediately from H.6.

In most cases Lemma H.4 implies that $\Theta_{0} L$ has a filtration with factors $Z_{\chi}(w \bullet \lambda, \alpha) \simeq L$ and $Z_{\chi}\left(w s_{0} \bullet \lambda, \alpha\right) \simeq Z_{\chi}\left(w s_{\alpha_{0}} \bullet \lambda, \alpha\right)$. The only exception occurs when $\left\langle w(\mu(0)+\rho), \alpha^{\vee}\right\rangle=\left\langle\mu(0)+\rho, \gamma^{\vee}\right\rangle$ is congruent to 0 modulo $p$. The choice of $\mu(0)$ implies that this can happen only if $\gamma=\alpha_{0}$, hence only if $R$ has type $A_{1}$. Set that case aside for the moment. We want to apply Lemma H.6 to $Z_{\chi}\left(w s_{\alpha_{0}} \bullet \lambda, \alpha\right)$; we have

$$
\left(w s_{\alpha_{0}}\right)^{-1} \alpha^{\vee}=s_{\alpha_{0}} \gamma^{\vee}=\gamma^{\vee}-\left\langle\alpha_{0}, \gamma^{\vee}\right\rangle \alpha_{0}^{\vee} .
$$

Since $\alpha_{0}^{\vee}$ is the largest root in $R^{\vee}$ and since $R$ is not of type $A_{1}$, we have $\left\langle\alpha_{0}, \gamma^{\vee}\right\rangle \in$ $\{0,1\}$. It follows that $\left(w s_{\alpha_{0}}\right)^{-1} \alpha<0$ if and only if $\left\langle\alpha_{0}, \gamma^{\vee}\right\rangle=1$. Now the claim concerning $\Theta_{0} L$ follows easily from H.6.

In the exceptional case $A_{1}$ we have $\gamma=\alpha_{0}=\alpha$ and $w=1$. In this case $T_{\lambda}^{\mu(0)} L \simeq Z_{\chi}(\mu(0))$. It follows that $\Theta_{0} L$ has a filtration with factors $Z_{\chi}(\lambda)$ and $Z_{\chi}\left(s_{0} \bullet \lambda\right) \simeq Z_{\chi}\left(s_{\alpha} \bullet \lambda\right)$. Both factors have length 2 with composition factors $L_{\alpha}^{\lambda}$ and $L_{0}^{\lambda}$. Again the claim follows since now $\left\langle\alpha_{0}, \gamma^{\vee}\right\rangle=2$.

When looking at $\Theta_{\gamma} L_{0}^{\lambda}$, then we may assume that there exists a simple root $\alpha$ and $w \in W$ with $w \alpha_{0}=-\alpha$ such that $\chi\left(\mathfrak{p}_{\alpha}\right)=0$ and $L \simeq Z_{\chi}(w \bullet \lambda, \alpha)$. One argues then as above. (The case $A_{1}$ requires a special argument as before.) 
H.9. Suppose in this subsection that all roots in $R$ have the same length and that $R$ is not of type $A_{1}$. Then the results in Lemma H.8 can be simplified using Theorem F.5. We get for all $\beta, \gamma \in \Pi$ with $\beta \neq \gamma$

$$
\left[\Theta_{\beta}^{\lambda} L_{\gamma}^{\lambda}\right]= \begin{cases}2\left[L_{\gamma}^{\lambda}\right]+\left[L_{\beta}^{\lambda}\right], & \text { if }(\beta, \gamma)<0 \\ 2\left[L_{\gamma}^{\lambda}\right], & \text { if }(\beta, \gamma)=0\end{cases}
$$

and for all $\gamma \in \Pi$

$$
\begin{aligned}
& {\left[\Theta_{0}^{\lambda} L_{\gamma}^{\lambda}\right]= \begin{cases}2\left[L_{\gamma}^{\lambda}\right]+\left[L_{0}^{\lambda}\right], & \text { if }\left(\alpha_{0}, \gamma\right)>0, \\
2\left[L_{\gamma}^{\lambda}\right], & \text { if }\left(\alpha_{0}, \gamma\right)=0,\end{cases} } \\
& {\left[\Theta_{\gamma}^{\lambda} L_{0}^{\lambda}\right]= \begin{cases}2\left[L_{0}^{\lambda}\right]+\left[L_{\gamma}^{\lambda}\right], & \text { if }\left(\alpha_{0}, \gamma\right)>0, \\
2\left[L_{0}^{\lambda}\right], & \text { if }\left(\alpha_{0}, \gamma\right)=0 .\end{cases} }
\end{aligned}
$$

By G.1, H.5, and H.7 the translation functors $T_{\lambda}^{\mu(\beta)}$ with $\beta \in \Pi \cup\{0\}$ take simple modules to simple modules or 0 ; if they take two simple modules $L_{1}$ and $L_{2}$ to isomorphic simple modules, then already $L_{1}$ and $L_{2}$ are isomorphic. The adjunction property of the translation functors implies that

$$
\operatorname{Hom}_{\mathfrak{g}}\left(\Theta_{\beta} M, M^{\prime}\right) \simeq \operatorname{Hom}_{\mathfrak{g}}\left(T_{\lambda}^{\mu(\beta)} M, T_{\lambda}^{\mu(\beta)} M^{\prime}\right)
$$

and

$$
\operatorname{Hom}_{\mathfrak{g}}\left(M^{\prime}, \Theta_{\beta} M\right) \simeq \operatorname{Hom}_{\mathfrak{g}}\left(T_{\lambda}^{\mu(\beta)} M^{\prime}, T_{\lambda}^{\mu(\beta)} M\right)
$$

for all modules $M, M^{\prime}$ in $\mathcal{C}_{\lambda}$. It follows that each $\Theta_{\beta} L_{\gamma}^{\lambda}$ with $\beta, \gamma \in \Pi \cup\{0\}$ has simple head and simple socle isomorphic to $L_{\gamma}^{\lambda}$. (These are standard arguments due to Vogan.) We have $\operatorname{soc} \Theta_{\beta} L_{\gamma}^{\lambda} \subset \operatorname{rad} \Theta_{\beta} L_{\gamma}^{\lambda}$. In case $\beta, \gamma \in \Pi$ we get now from $(1)$

$$
\operatorname{rad} \Theta_{\beta} L_{\gamma}^{\lambda} / \operatorname{soc} \Theta_{\beta} L_{\gamma}^{\lambda} \simeq \begin{cases}L_{\beta}^{\lambda}, & \text { if }(\beta, \gamma)<0, \\ 0, & \text { if }(\beta, \gamma)=0 .\end{cases}
$$

One gets similar formulas from (2) and (3).

Note that (6) implies in case $(\beta, \gamma)=0$ that we have a non-split extension

$$
0 \rightarrow L_{\gamma}^{\lambda} \longrightarrow \Theta_{\beta} L_{\gamma}^{\lambda} \longrightarrow L_{\gamma}^{\lambda} \rightarrow 0
$$

Let me write Ext $^{i}$ for Ext groups in the category of $U_{\chi}(\mathfrak{g})$-modules. So (7) says that $\operatorname{Ext}^{1}\left(L_{\gamma}^{\lambda}, L_{\gamma}^{\lambda}\right) \neq 0$ for all $\gamma \in \Pi$ such that there exists a simple root $\beta$ with $(\beta, \gamma)=0$. Using $(2)$ one gets similarly $\operatorname{Ext}^{1}\left(L_{\gamma}^{\lambda}, L_{\gamma}^{\lambda}\right) \neq 0$ in case $\left(\alpha_{0}, \gamma\right)=0$, and from $(3)$ one gets $\operatorname{Ext}^{1}\left(L_{0}^{\lambda}, L_{0}^{\lambda}\right) \neq 0$ if there exists a simple root $\gamma$ with $\left(\alpha_{0}, \gamma\right)=0$. This shows that $\operatorname{Ext}^{1}(L, L) \neq 0$ for all simple modules in $\mathcal{C}_{\lambda}$ unless $R$ has type $A_{2}$ (compatibly with Remark 1 in [J2], 2.19) or type $D_{4}$ where $L=L_{\alpha_{2}}^{\lambda}$ is the only possible exception. I have no idea how large these non-vanishing Ext groups are.

Proposition. Suppose that all roots in $R$ have the same length and that $R$ is not of type $A_{1}$. If $\beta$ and $\gamma$ are simple roots with $\beta \neq \gamma$, then

$$
\operatorname{Ext}^{1}\left(L_{\gamma}^{\lambda}, L_{\beta}^{\lambda}\right) \simeq \begin{cases}K, & \text { if }(\beta, \gamma)<0, \\ 0, & \text { if }(\beta, \gamma)=0 .\end{cases}
$$


If $\gamma$ is a simple root, then

$$
\operatorname{Ext}^{1}\left(L_{\gamma}^{\lambda}, L_{0}^{\lambda}\right) \simeq \operatorname{Ext}^{1}\left(L_{0}^{\lambda}, L_{\gamma}^{\lambda}\right) \simeq \begin{cases}K, & \text { if }\left(\alpha_{0}, \gamma\right)>0, \\ 0, & \text { if }\left(\alpha_{0}, \gamma\right)=0 .\end{cases}
$$

Proof. This follows again from standard arguments due to Vogan. When dealing with (8), one applies the functor $\operatorname{Hom}_{\mathfrak{g}}\left(, L_{\beta}^{\lambda}\right)$ to the short exact sequence

$$
0 \rightarrow \operatorname{rad} \Theta_{\beta} L_{\gamma}^{\lambda} \longrightarrow \Theta_{\beta} L_{\gamma}^{\lambda} \longrightarrow L_{\gamma}^{\lambda} \rightarrow 0 .
$$

The adjointness of $T_{\lambda}^{\mu(\beta)}$ and $T_{\mu(\beta)}^{\lambda}$ implies that $\Theta_{\beta}$ is self-adjoint. This implies

$$
\operatorname{Ext}^{i}\left(\Theta_{\beta} L_{\gamma}^{\lambda}, L_{\beta}^{\lambda}\right) \simeq \operatorname{Ext}^{i}\left(L_{\gamma}^{\lambda}, \Theta_{\beta} L_{\beta}^{\lambda}\right)=0
$$

for all $i \geq 0$; see H.8(2). It follows that

$$
\operatorname{Ext}^{1}\left(L_{\gamma}^{\lambda}, L_{\beta}^{\lambda}\right) \simeq \operatorname{Hom}_{\mathfrak{g}}\left(\operatorname{rad} \Theta_{\beta} L_{\gamma}^{\lambda}, L_{\beta}^{\lambda}\right) .
$$

Now apply (6).

The proof of (9) is similar, working with (2) and (3) instead of (1).

Remark. If $R$ has type $A_{1}$ and if we denote the unique simple root by $\gamma$, then one has

$$
\left[\Theta_{0}^{\lambda} L_{\gamma}^{\lambda}\right]=\left[\Theta_{\gamma}^{\lambda} L_{0}^{\lambda}\right]=2\left[L_{\gamma}^{\lambda}\right]+2\left[L_{0}^{\lambda}\right]
$$

It is well known that in this case

$$
\operatorname{Ext}^{1}\left(L_{\gamma}^{\lambda}, L_{0}^{\lambda}\right) \simeq \operatorname{Ext}^{1}\left(L_{0}^{\lambda}, L_{\gamma}^{\lambda}\right) \simeq K^{2} .
$$

(Note that $\chi=0$ and look at $[\mathrm{P}]$.)

H.10. We now want to extend some results from H.9 to the cases with two root lengths. Recall that we exclude type $G_{2}$. Note first that H.9(4) and H.9(5) hold without restriction. We next look at H.9(7).

Lemma. Let $\gamma$ be a simple root and let $L$ be a simple module in $\mathcal{C}_{\lambda}$ with $\kappa(L)=\{\gamma\}$. If $\beta$ is a simple root with $(\beta, \gamma)=0$ or if $\beta=0$ and $\left(\alpha_{0}, \gamma\right)=0$, then we have a short exact sequence

$$
0 \rightarrow L \longrightarrow \Theta_{\beta} L \longrightarrow L \rightarrow 0
$$

that does not split.

Proof. Lemma H.8 shows that $\Theta_{\beta} L$ has length 2 with both composition factors, say $L_{1}$ and $L_{2}$, satisfying $\kappa\left(L_{1}\right)=\kappa\left(L_{2}\right)=\{\gamma\}$. We want to show that $L_{1} \simeq L_{2} \simeq L$. If so, then we get a short exact sequence as in (1). If that sequence splits, then we get dim $\operatorname{Hom}_{\mathfrak{g}}\left(L, \Theta_{\beta} L\right)=2$ contradicting H.9(5).

If $\gamma$ is short, then $L_{1} \simeq L_{2} \simeq L$ follows from Theorem F.5. So consider the case where $\gamma$ is long. We may assume that there exists a simple root $\alpha$ with $\chi\left(\mathfrak{p}_{\alpha}\right)=0$ and $w \in W$ with $w \gamma=\alpha$ and $L \simeq Z_{\chi}(w \bullet \lambda, \alpha)$. The proof of Lemma H.8 shows that our claim will follow if we can show that $Z_{\chi}(w s \bullet \lambda, \alpha)$ is isomorphic to $L$ where $s=s_{\beta}$ in case $\beta \in \Pi$ and $s=s_{\alpha_{0}}$ in case $\beta=0$. We distinguish two cases:

The type of $R$ is $B_{n}$. We may assume that we are in the situation of [J2], Section 3. In particular, we have $\alpha=\alpha_{1}$ and $\gamma=\alpha_{j}$ with $1 \leq j<n$. We get from [J2], 3.13 that

$$
Z_{\chi}(w \cdot \lambda, \alpha) \simeq L_{\chi}\left(\lambda_{j}\right) \Longleftrightarrow w^{-1} \varepsilon_{1}=\varepsilon_{j} \text { and } w^{-1} \varepsilon_{2}=\varepsilon_{j+1}
$$


while

$$
Z_{\chi}(w \cdot \lambda, \alpha) \simeq L_{\chi}\left(\lambda_{2 n-j}\right) \Longleftrightarrow w^{-1} \varepsilon_{1}=-\varepsilon_{j+1} \text { and } w^{-1} \varepsilon_{2}=-\varepsilon_{j} .
$$

We have to show that $w s$ has the same property as $w$, i.e., that $s \varepsilon_{j}=\varepsilon_{j}$. The possibilities for $s$ are $s=s_{\varepsilon_{i}-\varepsilon_{i+1}}$ (with $i \neq j, j-1$ ) or $s_{\varepsilon_{n}}$ (if $j<n-1$ ) or $s_{\varepsilon_{1}}$ (if $j>1$ ). So we get indeed $s \varepsilon_{j}=\varepsilon_{j}$ and the claim follows in type $B_{n}$.

The type of $R$ is $C_{n}$ or $F_{4}$. We may assume that we are in the situation of F.6(1),(2) or F.6(3)-(5). We have to show that $w s \in W_{2} w$ in the notations from F.6(1) or F.6(3). Since $w s=s_{w \alpha^{\prime}} w$ with $\alpha^{\prime}=\beta$ or $\alpha^{\prime}=\alpha_{0}$ this means that $s_{w \alpha^{\prime}} \in W_{2}$. Now $\left(\alpha^{\prime}, \gamma\right)=0$ and $w \gamma=\alpha$ imply that $w \alpha^{\prime}$ is orthogonal to $\alpha$. So it suffices to show that $w \alpha^{\prime}$ is a short root. (Recall the definition of $W_{2}$.) That is obvious in case $\beta=0$. Otherwise note that in type $C_{n}$ or $F_{4}$ all simple roots orthogonal to a long simple root are short.

Remarks. 1) The lemma can be extended to the case where $L=L_{0}^{\lambda}$ and where $\beta$ is a simple root with $\left(\beta, \alpha_{0}\right)=0$.

2) We see as in $H .9$ that $\operatorname{Ext}^{1}(L, L) \neq 0$ in almost all cases. The only additional exceptions can occur in types $C_{2}=B_{2}$ and $C_{3}$.

H.11. Lemma. Let $\beta \in \Pi \cup\{0\}$, let $L$ be a simple module in $\mathcal{C}_{\lambda}$ with $\Theta_{\beta} L \neq 0$.

a) The head and the socle of $\Theta_{\beta} L$ are both isomorphic to $L$.

b) If $L^{\prime}$ is a simple module in $\mathcal{C}_{\lambda}$ with $T_{\lambda}^{\mu(\beta)} L^{\prime} \simeq T_{\lambda}^{\mu(\beta)} L$, then $L^{\prime} \simeq L$.

Proof. If $L^{\prime}$ is a simple module in $\mathcal{C}_{\lambda}$, then H.9(4),(5) imply that $L^{\prime}$ occurs with multiplicity 1 both in head and in the socle of $\Theta_{\beta} L$ if $T_{\lambda}^{\mu(\beta)} L^{\prime} \simeq T_{\lambda}^{\mu(\beta)} L$. Otherwise it does not occur at all. In particular $L$ itself occurs with multiplicity 1 . This shows that a) will follow from $b$ ).

Suppose that $L^{\prime}$ is a simple module in $\mathcal{C}_{\lambda}$ with $T_{\lambda}^{\mu(\beta)} L^{\prime} \simeq T_{\lambda}^{\mu(\beta)} L$. Lemma H.7 implies that $\kappa(L)=\kappa\left(L^{\prime}\right)$. If $L^{\prime}$ is not isomorphic to $L$, then there has to be a long simple root $\gamma$ with $\kappa(L)=\{\gamma\}$. Lemma H.8 shows that both $L$ and $L^{\prime}$ occur with multiplicity 1 as composition factors in $\Theta_{\beta} L$. Since they occur both in the head and in the socle they have to be direct summands. The discussion above of the socle implies that there cannot be any other contributions to the socle, hence that $\Theta_{\beta} L \simeq L \oplus L^{\prime}$. Then Lemma H.8 implies that $(\beta, \gamma)=0$ in case $\beta \in \Pi$, resp. $\left(\alpha_{0}, \gamma\right)=0$ in case $\beta=0$. Now Lemma H.10 yields a contradiction.

H.12. Proposition. a) If $\beta$ and $\gamma$ are simple roots with $(\beta, \gamma)=0$, then we have $\operatorname{Ext}^{1}\left(L, L^{\prime}\right)=0$ for all simple modules $L$ and $L^{\prime}$ in $\mathcal{C}_{\lambda}$ with $\kappa(L)=\{\gamma\}$ and $\kappa\left(L^{\prime}\right)=\{\beta\}$.

b) Let $\beta, \gamma$ be simple roots with $(\beta, \gamma)<0$, let $L$ be a simple module in $\mathcal{C}_{\lambda}$ with $\kappa(L)=\{\gamma\}$. Then there exists a simple module $L^{\prime}$ in $\mathcal{C}_{\lambda}$ with $\kappa\left(L^{\prime}\right)=\{\beta\}$ and

$$
\operatorname{Ext}^{1}\left(L, L^{\prime}\right) \simeq K \simeq \operatorname{Ext}^{1}\left(L^{\prime}, L\right) .
$$

If $\beta$ and $\gamma$ have the same length, then the condition $\operatorname{Ext}^{1}\left(L, L^{\prime}\right) \neq 0$ determines $L^{\prime}$ up to isomorphism. If $\beta$ and $\gamma$ have different lengths, then (1) holds for all possible $L$ and $L^{\prime}$.

c) Let $\gamma$ be a simple root. If $R$ is not of type $A_{1}$, then

$$
\operatorname{Ext}^{1}\left(L, L_{0}^{\lambda}\right) \simeq \operatorname{Ext}^{1}\left(L_{0}^{\lambda}, L\right) \simeq \begin{cases}K, & \text { if }\left(\alpha_{0}, \gamma\right)>0 \\ 0, & \text { if }\left(\alpha_{0}, \gamma\right)=0\end{cases}
$$


for all simple modules $L$ in $\mathcal{C}_{\lambda}$ with $\kappa(L)=\{\gamma\}$.

Proof. a) and b) Consider simple modules $L$ and $L^{\prime}$ in $\mathcal{C}_{\lambda}$ with $\kappa(L)=\{\gamma\}$ and $\kappa\left(L^{\prime}\right)=\{\beta\}$. Arguing as in H.9 one gets

$$
\operatorname{Ext}^{1}\left(L, L^{\prime}\right) \simeq \operatorname{Hom}_{\mathfrak{g}}\left(\operatorname{rad} \Theta_{\beta} L, L^{\prime}\right) .
$$

If we use the short exact sequence

$$
0 \rightarrow L \longrightarrow \Theta_{\beta} L \longrightarrow \Theta_{\beta} L / \operatorname{soc} \Theta_{\beta} L \rightarrow 0
$$

and apply the functor $\operatorname{Hom}_{\mathfrak{g}}\left(L^{\prime},\right)$, we get similarly

$$
\operatorname{Ext}^{1}\left(L^{\prime}, L\right) \simeq \operatorname{Hom}_{\mathfrak{g}}\left(L^{\prime}, \Theta_{\beta} L / \operatorname{soc} \Theta_{\beta} L\right) .
$$

If $(\beta, \gamma)=0$, then H.8 shows that $L^{\prime}$ is not a composition factor of $\Theta_{\beta} L$; this implies now the claim in a).

Suppose that $(\beta, \gamma)<0$. If $\beta$ and $\gamma$ have the same length, then $\left\langle\beta, \gamma^{\vee}\right\rangle=-1$. Then H.8 and H.11 imply: Given $L$ as above there exists a simple module $L_{1}^{\prime}$ with $\kappa\left(L_{1}^{\prime}\right)=\{\beta\}$ and

$$
\operatorname{rad} \Theta_{\beta} L / \operatorname{soc} \Theta_{\beta} L \simeq L_{1}^{\prime} .
$$

Then (3) and (4) show that $L_{1}^{\prime}$ satisfies (1) while these Ext groups are 0 for any simple module $L_{2}^{\prime}$ with $\kappa\left(L_{2}^{\prime}\right)=\{\beta\}$ and $L_{2}^{\prime} \not L_{1}^{\prime}$.

Consider now the case where $\beta$ and $\gamma$ have different lengths. The claim in the proposition is symmetric in $\beta$ and $\gamma$. So we may assume that $\beta$ is short and $\gamma$ is long. So there is up to isomorphism only one choice for $L^{\prime}$ while there are one or two possibilities for $L$. We have $\left\langle\beta, \gamma^{\vee}\right\rangle=-1$ and $\left\langle\gamma, \beta^{\vee}\right\rangle=-2$. Lemma H.8 yields therefore

$$
\operatorname{rad} \Theta_{\beta} L / \operatorname{soc} \Theta_{\beta} L \simeq L^{\prime} .
$$

This implies that (1) holds for all possible $L$ and $L^{\prime}$.

c) The proof is similar and left to the reader.

Remark. Let $\beta, \gamma$ be simple roots with $(\beta, \gamma)<0$ such that $\beta$ is short and $\gamma$ is long. We know that there exists up to isomorphism only one simple module $L_{\beta}^{\lambda}$ in $\mathcal{C}_{\lambda}$ with $\kappa$ invariant $\{\beta\}$. Consider now $M=\operatorname{rad} \Theta_{\gamma} L_{\beta}^{\lambda} / \operatorname{soc} \Theta_{\gamma} L_{\beta}^{\lambda}$. Lemma H.8 tells us that $M$ has length 2 , with both factors having $\kappa$ invariant $\{\gamma\}$. Part b) of the proposition implies using (3) and (4) that each simple module $L$ in $\mathcal{C}_{\lambda}$ with $\kappa$ invariant $\{\gamma\}$ occurs with multiplicity 1 in the socle and in the head of $M$.

There are now two possibilities: If there are (as expected) two isomorphism classes (with representatives $L_{\gamma, 1}^{\lambda}$ and $L_{\gamma, 2}^{\lambda}$ ) of simple modules in $\mathcal{C}_{\lambda}$ with $\kappa$ invariant $\{\gamma\}$, then we can apply the discussion to $L=L_{\gamma, 1}^{\lambda}$ and to $L=L_{\gamma, 2}^{\lambda}$. It follows that $M \simeq L_{\gamma, 1}^{\lambda} \oplus L_{\gamma, 2}^{\lambda}$ in this case. If, however, there is only one such class, then $M$ has to be a non-split extension of $L$ by itself. So, as in other situations, it is desirable to show that the middle $M$ is completely reducible. 
Notations

\begin{tabular}{|c|c|c|c|}
\hline $\operatorname{Ad}(g)$ & B.13 & $h$ & D.4 \\
\hline$B^{+}$ & B.14 & $h_{\alpha}$ & A.1 \\
\hline$C_{0}, C_{0}^{\prime}$ & C.1 & $\operatorname{ind}_{I}$ & B. 6 \\
\hline$F(J)$ & D.2 & $m_{\beta}$ & D. 6 \\
\hline$G$ & A. 1 & $\operatorname{pr}_{\lambda}$ & B.1 \\
\hline$G_{I}$ & B. 6 & $s_{\alpha}, s_{\alpha, m}$ & A. 1 \\
\hline$J(\lambda)$ & D.5 & $\operatorname{sbm}(w, \lambda)$ & C.7 \\
\hline$K$ & A. 1 & $v_{f}$ & A. 2 \\
\hline$K_{f}$ & A. 2 & $w_{0}$ & C. 4 \\
\hline$L_{0}^{\lambda}, L_{\alpha}^{\lambda}, L_{\alpha, i}^{\lambda}$ & G.1 & $x_{\alpha}$ & A. 1 \\
\hline$L_{\chi, \alpha}(f)$ & A. 3 & $y_{\alpha}$ & H.1 \\
\hline$M^{\beta}$ & C. 12 & $\mathcal{C}_{\lambda}$ & B. 1 \\
\hline$N$ & C. 5 & $\mathcal{C}(I)_{\lambda}$ & B. 6 \\
\hline$P_{I}$ & B. 6 & $\mathcal{C}(\chi), \mathcal{C}(\chi)_{\lambda}$ & B.13 \\
\hline$P_{\alpha}$ & B.14 & $\mathcal{P}, \mathcal{P}^{\prime}, \mathcal{P}_{\lambda}^{\prime}$ & G.3 \\
\hline$Q_{L}$ & B. 12 & $\mathfrak{b}^{+}$ & A. 1 \\
\hline$R, R^{+}$ & A.1 & $\mathfrak{g}, \mathfrak{g}_{\alpha}$ & A. 1 \\
\hline$R_{I}$ & B. 6 & $\mathfrak{g}_{I}$ & B. 6 \\
\hline$T$ & A.1 & $\mathfrak{h}$ & A. 1 \\
\hline$T_{\lambda}^{\mu}$ & B.2 & $\mathfrak{n}^{+}, \mathfrak{n}^{-}$ & A.1 \\
\hline$\hat{T(I)_{\lambda}^{\mu}}$ & B.7 & $\mathfrak{p}_{\alpha}$ & A. 3 \\
\hline$U(\mathfrak{g}), U_{\chi}(\mathfrak{g})$ & A.1 & $\mathfrak{p}_{I}$ & B. 6 \\
\hline$W, W_{p}$ & A.1 & $\Lambda_{\chi}$ & A. 2 \\
\hline$W_{I}$ & B. 6 & $\hat{\Theta_{\alpha}}$ & H. 8 \\
\hline$X$ & A.1 & $\alpha_{0}$ & D. 4 \\
\hline$Z_{\chi}(f)$ & A. 2 & $\kappa$ & D.5 \\
\hline$Z_{\chi, \alpha}(f)$ & A. 3 & $\varpi_{\beta}$ & D.2 \\
\hline$Z_{\chi, I}(\mu)$ & B. 6 & $\rho$ & A.1 \\
\hline$Z_{\chi}(f, \alpha)$ & A. 4 & $\varphi_{f}$ & A. 3, B. 8 \\
\hline$Z_{\chi}\left(f, \mathfrak{g}^{\prime}\right), Z_{\chi}\left(f, \alpha, \mathfrak{g}^{\prime}\right)$ & A. 5 & $\varphi_{\alpha}^{f}$ & A. 4, B. 10 \\
\hline \multirow[t]{2}{*}{$Z(f, \chi, \alpha)$} & A. 6 & $\varphi_{w}^{\lambda}$ & C. 6 \\
\hline & & $\chi(f)$ & A. 2 \\
\hline
\end{tabular}

\section{REFERENCES}

[Bou] N. Bourbaki, Groupes et algèbres de Lie, Chap. 4, 5 et 6, Hermann, Paris, 1968. MR 39:1590

[BG] K. A. Brown and I. Gordon, The ramification of centres: Lie algebras in positive characteristic and quantised enveloping algebras, preprint, April 1999.

[C] R. W. Carter, Finite Groups of Lie Type: Conjugacy Classes and Complex Characters, Wiley, Chichester, 1985. MR 87d:20060

[D] J. Dixmier, Algèbres enveloppantes, Gauthier-Villars, Paris, 1974. MR 58:16803a

[F] J. Franklin, Homomorphisms between Verma modules in characteristic p, J. Algebra 112 (1988), 58-85. MR 89c:17015

[FP1] E. M. Friedlander, B. J. Parshall, Modular representation theory of Lie algebras, Amer. J. Math. 110 (1988), 1055-1093. MR 89j:17015

[FP2] E. M. Friedlander, B. J. Parshall, Deformations of Lie algebra representations, Amer. J. Math. 112 (1990), 375-395. MR 91e:17012 
$[\mathrm{H}] \quad$ J. E. Humphreys, Modular representations of simple Lie algebras, Bull. Amer. Math Soc. (N.S.) 35 (1998), 105-122. MR 98k:17007

[J1] J. C. Jantzen, Representations of Algebraic Groups, (Pure and Applied Mathematics 131), Academic, Orlando, 1987. MR 89c:20001

[J2] J. C. Jantzen, Subregular nilpotent representations of $\mathfrak{s l}_{n}$ and $\mathfrak{s o}_{2 n+1}$, Math. Proc. Cambridge Philos. Soc. 126 (1999), 223-257. CMP 99:07

[J3] J. C. Jantzen, Representations of Lie algebras in prime characteristic (A. Broer, ed.), Representation Theories and Algebraic Geometry, Proceedings Montréal 1997 (NATO ASI series C 514), Kluwer, Dordrecht, 1998, pp. 185-235. MR 99h:17026

[J4] J. C. Jantzen, Modular representations of reductive Lie algebras, J. Pure Appl. Algebra (to appear).

[KW] V. Kac, B. Weisfeiler, Coadjoint action of a semi-simple algebraic group and the center of the enveloping algebra in characteristic p, Indag. Math. 38 (1976), 136-151. MR 54:5364

[L1] G. Lusztig, Periodic W-graphs, Represent. Theory 1 (1997), 207-279. MR 99a:20042

[L2] G. Lusztig, Bases in equivariant K-theory, Represent. Theory 2 (1998), 298-369. CMP 98:16

[L3] G. Lusztig, Subregular elements and bases in K-theory, Canad. J. Math. (to appear).

[L4] G. Lusztig, Representation theory in characteristic $p$, Lecture at the Taniguchi conference, Nara 1998, preprint, December 1998.

[L5] G. Lusztig, Bases in equivariant K-theory II, preprint, March 1999.

[P] R. D. Pollack, Restricted Lie algebras of bounded type, Bull. Amer. Math Soc. 74 (1968), 326-331. MR 36:2661

[Sl] P. Slodowy, Simple Singularities and Simple Algebraic Groups, Lecture Notes in Mathematics 815, Springer, Berlin, 1980. MR 82g:14037

[SpSt] T. A. Springer and R. Steinberg, Conjugacy classes, A. Borel et al, Seminar on Algebraic Groups and Related Finite Groups (Lecture Notes in Mathematics 131), Springer, Berlin, 1970, pp. 167-266. MR 42:3091

Matematisk Institut, Aarhus Universitet, Ny Munkegade, DK-8000 Aarhus C, DenMARK

E-mail address: jantzen@imf.au.dk 